Aus der Klinik für Thorax-, Herz- und Gefäßchirurgie (Prof. Dr. med. Dipl.- Phys. F.A. Schöndube) im Zentrum Chirurgie der Medizinischen Fakultät der Universität Göttingen

\title{
Über den Einfluss von Operationen mit Herz-Lungen-Maschine auf das postoperative Risiko, ein akutes Nierenversagen zu entwickeln
}

\author{
Inaugural-Dissertation \\ zur Erlangung des Doktorgrades \\ der Medizinischen Fakultät \\ der Georg-August-Universität zu Göttingen
}

vorgelegt von

Martin Maus

aus München

Göttingen 2014 
Dekan:

1.Berichterstatter:

2.Berichterstatter/in:

Tag der mündlichen Prüfung:
Prof. Dr.rer. nat. H.K. Kroemer

Prof. Dr. med. Dipl.- Phys. F.A. Schöndube

Priv. Doz. Dr. Daniel Patschan

20.05.2014 


\section{Inhaltsverzeichnis}

Inhaltsverzeichnis $\quad 1$

1 Einleitung $\quad 1$

1.1 Darstellung des Forschungsinhalts 1

1.2 Anatomie und Physiologie der gesunden Niere 2

1.2.1 Für das ANV relevante anatomische Besonderheiten 4

I. Nephron 4

II. Juxtaglomerulärer Apparat $\quad 6$

III. Durchblutung bzw. Gefäßversorgung der Niere 6

$\begin{array}{lll}\text { 1.2.2 Für das ANV relevante physiologische Besonderheiten } & 7\end{array}$

$\begin{array}{lll}\text { I. Autoregulation } & 7\end{array}$

II. Tubuloglomeruläres Feedback $\quad 8$

III. Renin-Angiotensin-Aldosteron-System 8

1.3 Akutes Nierenversagen (ANV) 9

$\begin{array}{lll}\text { 1.3.1 Definition/Inzidenz } & 9\end{array}$

I. Forschungsgeschichtliche Anmerkung 9

II. Definition des ANV 9

$\begin{array}{lr}\text { III. RIFLE-Kriterien } & 10\end{array}$

IV. Inzidenz des ANV 11

$\begin{array}{lll}\text { 1.3.2 Ätiologie des ANV } & 11\end{array}$

I. Prärenales ANV 12

II. Renales / intrinsisches ANV 13

$\begin{array}{ll}\text { III. Postrenales ANV } & 14\end{array}$

$\begin{array}{lll}\text { 1.3.3 Pathophysiologie des ANV } & 14\end{array}$

$\begin{array}{ll}\text { 1.3.4 Klinischer Verlauf des ANV } & 16\end{array}$

I. Initiale Schädigungsphase 16

$\begin{array}{lr}\text { II. Aufrechterhaltungsphase } & 16\end{array}$

$\begin{array}{ll}\text { III. Genesungsphase } & 17\end{array}$

$\begin{array}{lll}\text { 1.3.5 Diagnose des ANV } & 17\end{array}$

$\begin{array}{ll}\text { I. Klinik des pärenalen ANV } & 18\end{array}$

$\begin{array}{lr}\text { II. Labor } & 19\end{array}$

III. Urinuntersuchung, Sediment, Urinindizes 21

$\begin{array}{lll}\text { 1.3.6 Komplikationen des ANV } & 23\end{array}$

$\begin{array}{ll}\text { I. Erhöhung des Extrazellurärvolumens } & 23\end{array}$

$\begin{array}{ll}\text { II. Hyperkaliämie } & 23\end{array}$

III. Metabolische Azidose 24

IV. Infektionen 24

V. Urämisches Syndrom 24

1.3.7 Therapiemöglichkeiten des ANV 25

I. Prävention 25

II. Spezifische konservative Therapie 26

$\begin{array}{ll}\text { III. Nierenersatzverfahren } & 27\end{array}$

$\begin{array}{ll}\text { 1.3.7.1 Nierenersatztherapie beim ANV } & 30\end{array}$

$\begin{array}{lll}\text { 1.3.8 Prognose des ANV } & 31\end{array}$

1.4 Stand der Forschung zum ANV nach kardiochirurgischen Eingriffen 32 
2 Methodik $\quad 37$

$2.1 \quad$ Patientengruppe 37

2.2 Datensatz 39

2.3 Statistik 42

3 Ergebnisse $\quad 45$

3.1 Demographische Daten $\quad 45$

3.1.1 Geschlecht der Patienten $\quad 45$

3.1.2 Alter der Patienten 46

3.2 Operationen 48

3.2.1 Gesamtmortalität 48

3.3 Patienten mit Einsatz eines Nierenersatzverfahrens (NEV) 49

3.3.1 Mortalität der Patienten mit NEV 49

3.4 Inzidenz des ANV gemäß RIFLE-Kriterien $\quad 50$

3.4.1 Mortalität der Patienten mit ANV $\quad 50$

3.5 Einfluss der kategoriellen Parameter auf die Indikation eines NEV 51

3.6 Einfluss der metrischen Parameter auf die Indikation eines NEV 56

3.7 Blutverlust und NEV 59

3.8 Einfluss der kategoriellen Parameter auf das Auftreten eines ANV gemäß RIFLE 62

3.9 Einfluss der metrischen Parameter auf das Auftreten eines ANV gemäß RIFLE 66

3.10 Einfluss der kategoriellen Parameter auf den absoluten Kreatitininanstieg $\quad 68$

3.11 Einfluss der metrischen Parameter auf den absoluten Kreatininanstieg 69

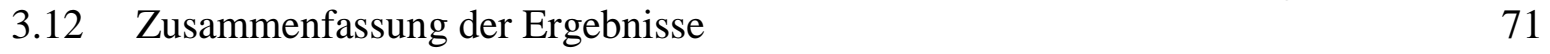

4 Diskussion $\quad \mathbf{7 3}$

4.1 Allgemeine Aspekte $\quad 73$

4.1.1 Gesichtspunkte zur Inzidenz / Mortalität / Definition des ANV 73

4.1.2 Inzidenz / Mortalität unserer Studie im Kontext der Literatur 75

4.1.3 ANV als multifaktorielles Geschehen $\quad 76$

4.2 Beziehung der Ergebnisse zum aktuellen Forschungsstand 78

$\begin{array}{lll}\text { 4.2.1 Personen-und Operations-assoziierte Parameter } & 78\end{array}$

$\begin{array}{ll}\text { 4.2.2 HLM-assoziierte Parameter } & 82\end{array}$

5 Zusammenfassung $\quad 88$

6 Literaturverzeichnis 91

7 Anhang $\quad 97$

$\begin{array}{lll}7.1 & \text { Tabellenverzeichnis } & 97\end{array}$

$\begin{array}{lll}7.2 & \text { Abbildungsverzeichnis } & 98\end{array}$

$\begin{array}{lll}7.3 & \text { Abkürzungsverzeichnis } & 99\end{array}$ 


\section{Einleitung}

\subsection{Darstellung des Forschungsinhalts}

Das akute Nierenversagen (ANV) nach herzchirurgischen Operationen ist auch heute noch von besonderer Bedeutung, da seine Behandlung schwierig, komplikationsträchtig, kostenintensiv und vor allem mit einer hohen Mortalität belastet ist (Garwood 2004). In den letzten 40-50 Jahren haben sich die Resultate hinsichtlich des Risikos, ein ANV zu entwickeln, für die Patienten trotz verbesserten chirurgischen Techniken wie beispielsweise der Bypasschirurgie am schlagenden Herzen oder neuen Kathetern, Pharmazeutika in der Kardiologie oder neuer Dialysetechnologie bzw. der Einführung der biokompatiblen Dialysemembranen nicht grundlegend verbessert. Grund dafür könnte einerseits die Möglichkeit der Behandlung noch älterer, mit mehr Grundleiden belasteter Patienten sein, welche durch verbesserte Methoden einer Operation am Herzen zugänglich gemacht werden. Aber auch die wesentlich komplexere Pathophysiologie der Entstehung eines ANV und der mit ihr verbundenen Konsequenzen scheint ein Grund dafür zu sein, dass einige vielversprechende Ansätze der Behandlung, wie die niedrig dosierte Dopamingabe oder Diuretika nicht den erwarteten Erfolg zeigten (Garwood 2004). Es deutet einiges darauf hin, dass in dem komplexen Entstehungsmechanismus des ANV Faktoren involviert sind, welche speziell die Herz-Lungen-Maschine und deren Komponenten betrifft. Herauszufinden, inwieweit diese Parameter einen signifikanten Zusammenhang mit der Entstehung eines ANV haben, war Anliegen unserer Untersuchungen.

Zu diesem Zweck wurde anhand der Aufarbeitung und retrospektiven Analyse von Patientendaten von 3574 herzchirurgischen Patienten aus den Jahren 2000-2005 aus mehreren Datenbanken des Universitätsklinikums Göttingen untersucht, wie ausgewählte perioperative Parameter mit dem Auftreten eines akuten Nierenversagens assoziiert sind. Neben allgemeinen Variablen wie Alter oder Geschlecht und operationsassoziierten Variablen wie der Art der Operation oder dem Einsatz einer IABP (Intraaortale Ballonpumpe) waren dabei die intraoperativen Einflussgrößen, die in Zusammenhang mit der extrakorporalen Zirkulation stehen, von besonderem Interesse. Insbesondere zu nennen sind hier die Operationsdauer, Ischämiezeit, Perfusionszeit, Reperfusionszeit und der Grad der Hypothermie. Ein weiterer Schwerpunkt der Analyse war die Untersuchung der Blutverluste in absolutem Maße, aber auch im Zeitverlauf und deren Auswirkung auf das Auftreten eines ANV. 
Der Definition des ANV wurden dabei die neuesten Richtlinien zugrunde gelegt und daher neben der Analyse aller Parameter bezüglich des Bedarfs eines Nierenersatzverfahrens auch deren Untersuchung im Hinblick auf ein ANV gemäß der Definition der RIFLE-Kriterien R = Risk (Risiko), I = Injury (Schädigung), F = Failure (Versagen), L = Loss (Verlust) und E = ESKD $=$ End-Stage Kidney Disease (Terminales Nierenversagen) (Venkataraman und Kellum 2007) vorgenommen. Diese Klassifikation ist seit dem Jahr 2004 zum internationalen Standard für die Definition eines Nierenversagens geworden. Dementsprechend ist es allerdings auch schwierig, Ergebnisse in den Kontext älterer Literatur zu stellen, da vor diesem Konsensus in fast jeder wissenschaftlichen Arbeit eine andere Definition des ANV verwendet wurde.

\subsection{Anatomie und Physiologie der gesunden Niere}

Die Niere ist ein lebenswichtiges Organ, welches aufgrund seines komplexen anatomischen Aufbaus eine erstaunliche Regenerationskapazität bei nur temporärer Schädigung - wie z.B. einer Minderperfusion - besitzt. Bei prolongierten schädigenden Einflüssen entsteht allerdings eine irreversible Organschädigung mit weitreichenden Auswirkungen für den Organismus.

Die gesunde Niere bestimmt maßgeblich die Aufrechterhaltung der Volumen- bzw. Elektrolythomöostase und die Ausscheidung von wasserlöslichen Stoffwechselprodukten sowie harnpflichtigen Substanzen wie Kreatinin, Harnstoff, Ammoniak oder Harnsäure. Das grundlegende Prinzip hierbei ist die Filtration eines großen Flüssigkeitsvolumens aus dem Blut in die Tubuli. Die Filtration findet in den Nierenkörperchen statt, welche aus dem Kapillarknäuel, aus dem die Flüssigkeit ausgepresst wird und der Bowmanschen Kapsel, in der der Primärharn aufgefangen und in den Tubulus weitergeleitet wird, besteht. (siehe Abb.1) 


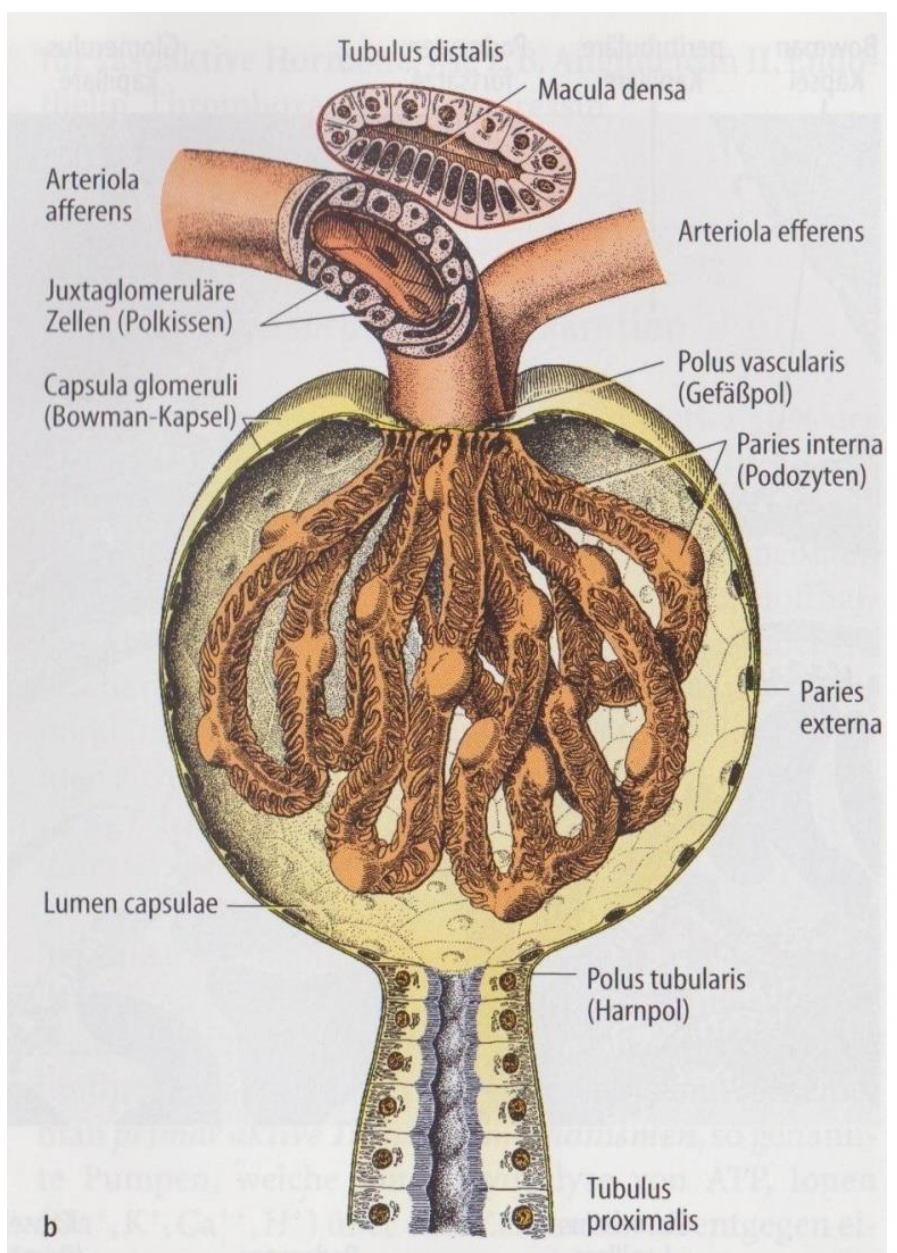

Abb.1: Aufbau des Glomerulus (Junqueira 2002, S.329)

Der für die Filtration benötigte Druckgradient wird letztendlich vom Herzen aufgebaut, was die enge Beziehung zwischen Herzfunktion und Nierenfunktion bereits andeutet. Anschließend findet in Tubulus und Sammelrohr eine bedarfsgerechte Reabsorption von Substanzen des Primärharns wie z.B. Glukose, Elektrolyte und Wasser statt. Für die Regulierung des Wasserhaushaltes spielt dabei das Antidiuretische Hormon (ADH) eine große Rolle. Im Kalium- und Natriumhaushalt spielt Aldosteron, welches die Rückresorption von Natrium und die Ausscheidung von Kalium steigert, eine bedeutende Rolle. Nicht rückresorbierte Bestandteile werden im Rahmen der Exkretion mit dem Urin ausgeschieden. Zusätzlich werden manche Moleküle in einem als Sekretion bezeichneten Vorgang aktiv in das Tubuluslumen transportiert, z.B. vorwiegend in proximalen Tubuli sezernierte organische Anionen wie einige Antibiotika, Diuretika, anti-retrovirale Pharmaka und Schmerzmittel. Auch an der Regulation des Säure-Base-Haushaltes ist die Niere entscheidend beteiligt, indem sie die Sekretion und Ausscheidung von Wasserstoff-Ionen und Hydronium-Ionen an die gegebenen Bedürfnisse im Organismus anpasst. Erythropoetin (EPO) wird überwiegend in 
der Niere produziert. Damit ist die Niere wichtigster EPO-Lieferant bei der Stimulation der Hämatopoese im Rahmen von verminderter Sauerstoffsättigung im Blut. In der Niere findet auch die Hydroxylierung von 1-Hydroxycholecalciferol zu 1,25-Dihydroxycholcalciferol, dem aktiven Metaboliten des Vitamin-D, statt. Dieser auch Calcitriol genannte aktive Metabolit erhöht die Absorption von Kalzium und Phosphat im Gastrointestinaltrakt und der Niere und inhibiert die Ausschüttung von Calcitonin. Im Rahmen des Renin-AngiotensinAldosteron-Systems, welches gesondert unter 1.2.2 erläutert wird, greift die Niere auf mehreren Ebenen in die Regulierung des Blutdrucks ein.

Die im Folgenden dargestellten anatomischen und physiologischen Grundlagen sind insbesondere wichtig zum Verständnis der Pathogenese des akuten Nierenversagens und der Faktoren, die dieses auslösen, verstärken oder mildern können.

\subsubsection{Für das ANV relevante anatomische Besonderheiten}

Zum Verständnis der Auswirkungen auf die Niere und deren Durchblutung während einer großen Operation am Herzen und der Pathophysiologie eines ANV sind einige grundlegende anatomische und physiologische Merkmale der Niere von großer Bedeutung. Im folgenden werden daher das Nephron als kleinste funktionelle Einheit der Niere, der juxtaglomeruläre Apparat als wichtige Schaltstation für die Blutdruckregulierung und die besondere Gefäßarchitektur der Niere kurz dargestellt.

\section{Nephron}

Als Nephron bezeichnet man die funktionelle Einheit der Niere, die aus dem

Nierenkörperchen, welches seinerseits aus Glomerulus und Bowmanscher Kapsel besteht und dem zugehörigen Nierentubulus aufgebaut ist (Frick et al. 1992). Filtrationsbarrieren auf dem Weg zum Primärharn sind dabei das fenestrierte Endothel der Kapillaren des Glomerulus, die Basalmembran und die Schlitzmembranen zwischen den Podozyten, welche aus spezialisierten viszeralen Epithelzellen bestehen. Die Filtration findet ausschließlich in den Nierenkörperchen statt und wird in ihrem Ausmaß von dem effektiven Filtrationsdruck $\left(\mathrm{P}_{\text {eff }}\right)$ bestimmt. Dieser ist abhängig von der hydrostatischen Druckdifferenz zwischen glomerulärer Kapillarschlinge $\left(\mathrm{P}_{\mathrm{cap}}\right)$ und Bowmanschem Kapselraum $\left(\mathrm{P}_{\mathrm{bc}}\right)$ und der Differenz des onkotischen Drucks zwischen Kapillare ( $\left.\mathrm{II}_{\mathrm{cap}}\right)$ und dem Bowmanschen Kapselraum $\left(\mathrm{II}_{\mathrm{bc}}\right)$. Dieser ist unbedeutend und somit zu vernachlässigen. Daraus ergibt sich: $\mathrm{P}_{\mathrm{eff}}=\mathrm{K}_{\mathrm{f}}\left(\mathrm{P}_{\mathrm{cap}}-\mathrm{P}_{\mathrm{bc}}-\right.$ $\left.\mathrm{II}_{\text {cap }}\right)$. Der Filtrationskoeffizient $\left(\mathrm{K}_{\mathrm{f}}\right)$ beschreibt wasserleitende Eigenschaften der Filtrationsbarriere (mündliche Mitteilung, Prof. Dr. Burckhardt, Abteilung vegetative Physiologie und Pathophysiologie, Georg-August-Universität Göttingen, 2003). Die 
glomeruläre Filtration hängt also vom Plasmafluss (Brenner et al. 1972), dem Blutdruck, welcher lokal durch Vas afferens und Vas efferens beeinflusst wird, der onkotisch wirksamen Zusammensetzung des Blutes und dem Filtrationskoeffizienten ab. Eine Reduktion der Anzahl oder Größe der Filtrationsporen durch Kontraktion von glomerulären Epithelzellen wird für eine mögliche Abnahme des Filtrationskoeffizienten verantwortlich gemacht (Andrews und Coffey 1983). Die glomeruläre Filtrationsrate (GFR) ist das von allen Glomeruli pro Zeiteinheit filtrierte Volumen. Sie beträgt je nach Indikatorsubstanz 120-145 ml/min (mündliche Mitteilung, Prof. Dr. Burckhardt, 2003). Jede Niere besteht aus ca. einer Millionen Nephronen. Die funktionelle Reservekapazität ist so groß, dass auch mit nur einer gesunden Niere eine normale Nierenfunktion aufrecht erhalten werden kann. Bei Entfernung einer Niere kann die Verbleibende im Rahmen einer kompensatorischen Hypertrophie auf nahezu das doppelte Volumen heranwachsen (Frick et al. 1992). Erst ab einer Reduktion der Anzahl funktionierender Nephrone um mehr als 60-70\% tritt eine Niereninsuffizienz ein (Krautzig 2004).

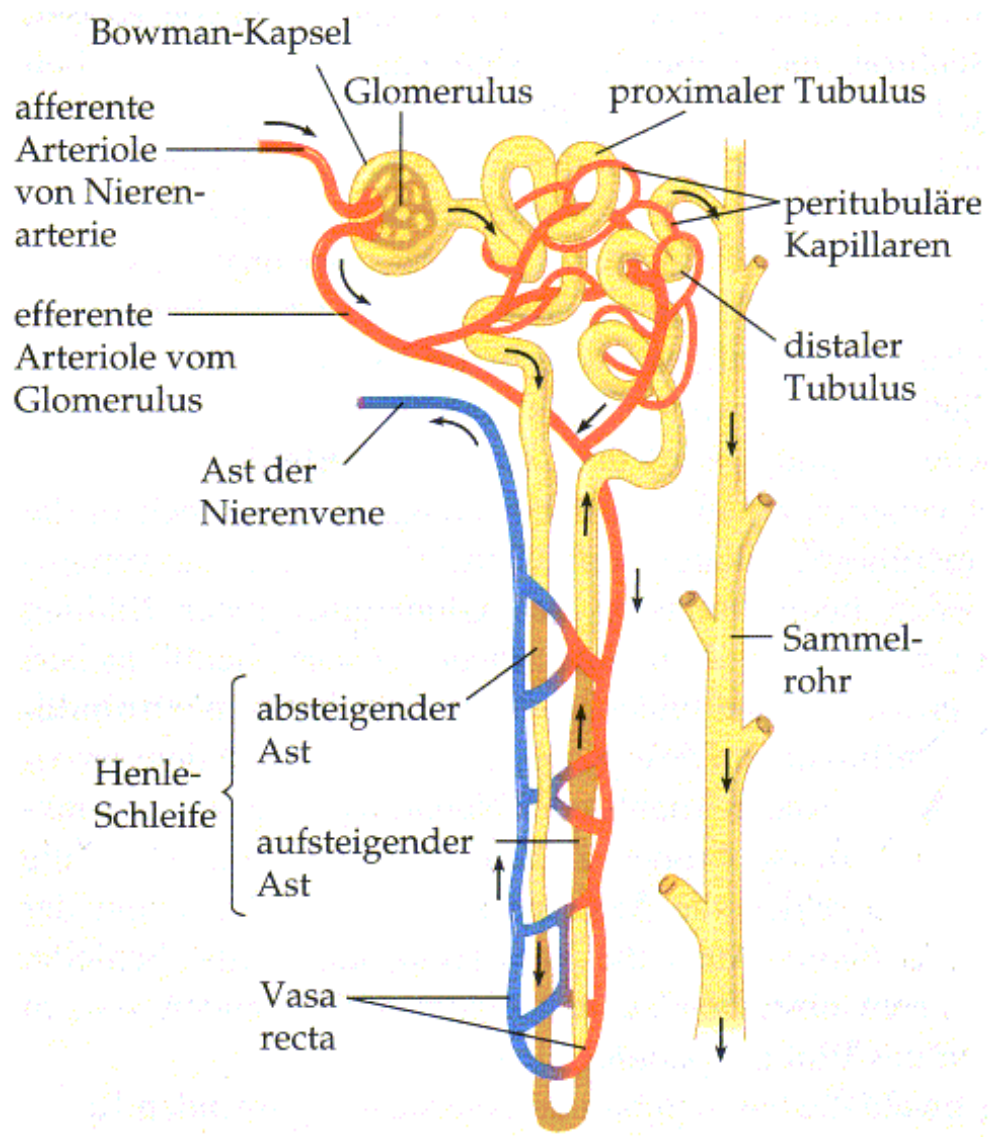

Abb. 2: Nephron (Silbernagel 2003, S. 148) 


\section{Juxtaglomerulärer Apparat}

Der juxtaglomeruläre Apparat besteht aus spezialisierten Zellen an der Verbindung des distalen Konvoluts, d.h. der Pars recta des distalen Tubulus und der Arteriola afferens des Glomerulus. Er besteht aus der Makula densa, einer Zellregion im distalen Tubulus, die sich von den anderen Tubuluszellen unterscheidet und gegenüber der Arteriola afferens liegt. Hier kann ein erniedrigter $\mathrm{NaCl}-G e h a l t$ des Intermediärharns detektiert werden und führt dann zur Freisetzung von Renin. Die juxtaglomerulären Zellen sind spezialisierte glatte Muskelzellen in der Media an der Endstrecke der Arteriola afferens, welche das Renin synthetisieren und sezernieren, aber auch selbst als Barorezeptoren den Blutdruck in der zuführenden Arteriole messen können und bei dessen Abfall mit Reninausschüttung reagieren. Stimuli für die Reninausschüttung sind demnach ein durch sympathische Nervenfasern vermittelter Abfall des systemischen Bluckdrucks, ein an der Makula densa detektierter verminderter $\mathrm{NaCl}$ Gehalt oder ein durch die am juxtaglomerulären Apparat befindlichen Barorezeptoren direkt vermittelter Blutdruckabfall. Die Wirkungen des Renins im Rahmen des Renin-AngiotensinAldosteron-Systems werden unter 1.2.2 genauer beschrieben. Des Weiteren finden am juxtaglomerulären Apparat die Prozesse des tubuloglomerulären Feedbacks statt, auf die unter 1.2.2 eingegangen wird.

\section{Durchblutung bzw. Gefäßversorgung der Niere}

Obwohl der Anteil des Sauerstoffverbrauchs der Nieren bezogen auf den gesamten Organismus nur 7\% beträgt, beanspruchen sie 20\% des Herzzeitvolumens (Silbernagel 2003). Das heißt, im Verhältnis zu ihrem geringen Anteil am Körpergewicht von ca. 0,5\% haben die Nieren eine sehr hohe Durchblutung. Der Grund dafür liegt in der Funktion der Niere als Ausscheidungsorgan, welches große Mengen Blut von Stoffwechselendprodukten befreien muss. Es erklärt aber auch, warum die Nierendurchblutung bei drohendem Schock vorübergehend zu Gunsten von Gehirn und Herz, welche auf Sauerstoffmangel besonders empfindlich reagieren, gedrosselt werden kann, ohne dass die Niere bleibenden Schaden davontragen muss (Silbernagel 2003). Die Gefäßversorgung der Niere ist aufgrund der besonderen Anforderungen, wie der Konzentrierung des Harns im Gegenstromsystem, speziell strukturiert und wird auch als Rete mirabile (lateinisch: "Wundernetz") bezeichnet. Gemeint ist damit die Hintereinanderschaltung von zwei Kapillarnetzen. In der Niere zweigen aus den Arteriae arcuatae, die zwischen Kortex und Medulla verlaufen, Arteriae interlobares ab, aus denen ihrerseits die Vasa afferentia entspringen. Diese Arteriolen führen bei relativ hohem Druck in das glomeruläre Kapillarnetz und münden dann wieder in Arteriolen, nämlich die Vasa efferentia. Das folgende Kapillarnetz umspinnt entweder die kortikalen 
Tubuli als peritubuläre Kapillaren oder steigt als Vasa recta tief ins Nierenmark ab. Letzteres ist der Fall bei juxtamedullären Nephronen. D.h., der tubuläre Blutfluss ist vollkommen postglomerulär und die Blutversorgung des Tubulusystems ist sehr eng mit der glomerulären Filtration verknüpft. Der Filtrationsdruck und damit die GFR wird maßgeblich durch die Regulierung von zu- und abführender Arteriole bestimmt (Lameire und Vanholder 1998). Diese Regulation der Nierendurchblutung wird durch viele Faktoren und Mediatoren beeinflusst, welche bei der Darstellung der Pathophysiologie des ANV im Abschnitt 1.3.3 genau beschrieben werden.

\subsubsection{Für das ANV relevante physiologische Besonderheiten}

Aufbauend auf dem Verständnis der oben aufgeführten anatomischen Merkmale der Niere sind für die Erklärung der Pathophysiologie des ANV einige physiologische Besonderheiten der Niere von besonderer Bedeutung.

\section{Autoregulation}

Der renale Plasmafluss und damit die GFR unterliegen einer Autoregulation. Das bedeutet, dass bei sich ändernden Blutdruckwerten zwischen $80-180$ mmHg die GFR relativ konstant bleibt, weil der Widerstand in den Arteriae interlobulares und Vasa afferentia dem herrschenden arteriellen Mitteldruck angepasst wird (Lameire und Vanholder 1998). Diese auch Bayliss-Effekt genannten Vorgänge bieten einen Schutz der glomerulären Kapillarschlingen bei zu hohen Blutdruckwerten, aber auch eine ausreichende glomeruläre Filtration bei niedrigen Blutdrücken. Bei einem Blutdruck unter $80 \mathrm{mmHg}$ sinkt die Durchblutung allerdings fortschreitend und die Filtration versiegt in gleichem Maß. Rein theoretisch könnten bei der Niere ähnliche Systeme - wie in anderen Organen mit Autoregulation - eine Rolle spielen wie myogene Regulation, metabolische Signaltransduktion oder interstitieller Druck. Aber man ist sich nicht sicher, welche die korrigierte Entität ist, Druck oder Durchfluss, und wie und wo das Signal wahrgenommen wird. Die Studie von Brezis und Rosen von 1995 besagt, dass die Durchblutung der Medulla von metabolischen Signalgebern beeinflusst wird und Davies et al. kommen $1988 \mathrm{zu}$ der Erkenntnis, dass endotheliale Strukturen an der Wahrnehmungsfunktion beteiligt sein müssen. Galle und Wanner beschreiben 1996, dass die Autoregulation der Niere durch die Blockade von Stickoxid (NO) stark abgeschwächt wird, aber nicht vollständig erlischt. 


\section{Tubuloglomeruläres Feedback}

Das tubuloglomeruläre Feedback ist eine weitere physiologische Anpassungsreaktion, die teilweise zur Autoregulationsfunktion der Niere beiträgt, aber diese nicht vollständig erklärt (Johnson 1986). Das tubuloglomeruläre Feedback gewährleistet eine Konstanthaltung der glomerulären Filtration des einzelnen Nephrons. Wenn bei steigender Filtration die Konzentration von $\mathrm{NaCl}$ im frühen distalen Tubulus wegen mangelnder Rückresorption steigt, wird dies von der Makula densa im juxtaglomerulären Apparat wahrgenommen und eine vom Mesangium vermittelte Vasokonstriktion der zuführenden Arteriole und eine Verminderung der Filtrationsfläche führen zum Abfall der Filtration. In diesem physiologischen Fall stellt diese Reaktion einen Schutz des Nephrons vor Hyperfiltration dar. Man ist sich einig, dass durchflussabhängige Veränderungen der Tubulusflüssigkeit das Signal darstellen, welches in der Makula densa wahrgenommen werden (Lameire und Vanholder 1998). Defizite der Widerstandsfähigkeit der zu- und abführenden Arteriole, welche unerlässlich für die Aufrechterhaltung der Nierendurchblutung, besonders im Rahmen eines low cardiac output sind, schränken sowohl die Autoregulation als auch tubuloglomerulären Feedback der Niere ein (Badr und Ichikawa 1988).

\section{Renin-Angiotensin-Aldosteron-System}

Auch das Renin-Angiotensin-Aldosteron-System ist von entscheidender Bedeutung für die Nierendurchblutung und -Funktion. Denn neben Aldosteron, welches direkt an der Niere angreift, hat auch Angiotensin II neben seiner systemischen auch spezifische Wirkungen an der Niere selbst. Angiotensin II vermittelt in der Niere die Konstriktion von glomerulären Mesangiumzellen und glatten Muskelzellen in Interlobärarterien und afferenten und efferenten Arteriolen (Lameire und Vanholder 1998). Zudem hat Angiotensin II eine direkte antinatriuretische Wirkung auf den proximalen Tubulus (Harris and Navar 1985). Die Reninausschüttung in der Niere wird bei einem systemischen Blutdruckabfall über arterielle Barorezeptoren, die dann über den Symphatikotonus $\beta_{1}$ adrenerge Rezeptoren in der Makula densa stimulieren, getriggert. Aber auch im juxtaglomerulären Apparat gibt es Barorezeptoren, die direkt die Reninsekretion stimulieren. Eine Minderdurchblutung der Niere äußert sich in einer verminderten glomerulären Filtration, was einen verminderten $\mathrm{NaCl}-\mathrm{Gehalt}$ bewirkt und, durch Detektion an der Makula densa, die Reninausschüttung stimuliert. Renin wandelt aus der Leber stammendes Angiotensinogen in Angiotensin I um, welches seinerseits durch das insbesondere im Gefäßendothel lokalisierte Angiotensinkonvertierende Enzym in Angiotensin II abgespalten wird. Systemisch bewirkt Angiotensin II als potenter Vasokonstriktor durch Erhöhung des Gefäßwiderstandes eine Blutdruckerhöhung. 
Zudem ist Angiotensin II der potenteste endogene Stimulator der Freisetzung von Aldosteron aus der Nebennierenrinde. Aldosteron ist ein Mineralokortikoid, das die Resorption von Natriumionen und damit die Flüssigkeitsretention steigert. Auch dadurch kommt es über das gesteigerte Blutvolumen zu einem Anstieg des arteriellen Blutdrucks (mündliche Mitteilung, Prof. Dr. Burckhardt, Georg-August-Universität Göttingen, 2003).

\subsection{Akutes Nierenversagen (ANV)}

\subsubsection{Definition/Inzidenz}

\section{Forschungsgeschichtliche Anmerkung}

Die erste detaillierte Beschreibung eines schweren akuten Nierenversagens geht auf den deutschen Pathologen Hackradt aus dem Jahr 1917 zurïck und basierte auf der Beobachtung schwer traumatisierter Soldaten. Seine Beschreibungen wurden abgestritten, bis das Syndrom der “Crush-Niere” bei Opfern des London Blitz (Luftangriffe auf London im 2. Weltkrieg) beschrieben wurde und seine Ansichten bestätigte (Lameire und Vanholder 1998). Ein Meilenstein bei der Behandlung solcher Patienten war die Einführung der Hämodialyse in den fünfziger Jahren, welche die Mortalität bei militärisch traumatisierten Patienten von $90 \%$ auf 5\% senkte (Lameire und Vanholder 1998). Konsequent weitergeführte Studien zeigten, dass akutes, potentiell reversibles Nierenversagen, welches in vielen Fällen mit histologischen Korrelaten einer akuten Tubulusnekrose einhergeht, auch unter anderen Umständen auftreten kann, wie z.B. bei einer Fehltransfusion von Blutbestandteilen, im Rahmen eines kardiogenen oder septischen Schocks oder aufgrund der Wirkung einer Reihe von nephrotoxischen Substanzen.

\section{Definition des ANV}

Laut Harrison et al. (2005) wird das akute Nierenversagen (ANV) als ein Syndrom definiert, das durch eine rasche Abnahme der glomerulären Filtrationsrate (Stunden bis Tage), die Retention von stickstoffhaltigen Abfallprodukten sowie durch eine gestörte Homöostase des extrazellulären Flüssigkeitsvolumens und des Elektrolyt- und Säurebasenhaushaltes gekennzeichnet ist. Eine Oligurie (Urinproduktion $<500 \mathrm{ml} / \mathrm{d}$ ) ist ein häufiges, aber nicht zwingendes klinisches Merkmal (etwa 50\%). Das ANV verläuft in der Anfangsphase meist asymptomatisch und wird erst beim biochemischen Monitoring von Krankenhauspatienten durch einen akuten Anstieg des Plasmaharnstoffs und Plasmakreatinins diagnostiziert. Meistens ist das ANV reversibel, denn die Nieren sind unter den größeren Organen in ihrer Fähigkeit einzigartig, sich vom fast vollständigen Funktionsverlust wieder zu erholen. Aber 
aufgrund der meist schwerwiegenden Grunderkrankungen, die das ANV mit ausgelöst haben, ist es mit einer hohen Krankenhausmorbidität und Mortalität belastet (Harrison et al. 2005).

\section{RIFLE-Kriterien}

In der Klinik hat sich zur Definition des ANV und seiner Stadieneinteilung die RIFLE-

Klassifikation durchgesetzt. Dabei steht RIFLE für R = Risk (Risiko), I = Injury

(Schädigung), F = Failure (Versagen), L = Loss (Verlust) und E = ESKD = End-Stage Kidney

Disease ( Terminales Nierenversagen) (Venkataraman und Kellum 2007). Diesem Schema, welches 2004 von einer internationalen Konsensuskonferenz entworfen wurde, liegen als unabhängige Kriterien das Serumkreatinin und die Urinausscheidung zugrunde. Die RIFLE Kriterien sind die ersten internationalen und interdisziplinären Kriterien für die Einteilung und Definition des ANV(Venkataraman und Kellum 2007). Auch in unserer Studie haben wir uns aufgrund der breiten Akzeptanz in Klinik und Forschung auf die RIFLE-Kriterien bezogen, um das ANV zu definieren. Um die den Stadien zugehörigen Kriterien übersichtlicher darzustellen, werden in Anlehnung an Venkataraman und Kellum (2007) die RIFLE-Kriterien noch einmal in Tab. 1 zusammengefasst:

\begin{tabular}{|c|c|c|}
\hline $\begin{array}{l}\text { RIFLE- } \\
\text { Kriterien }\end{array}$ & $\begin{array}{l}\text { Kriterien der glomerulären } \\
\text { Filtrationsrate (GFR) }\end{array}$ & $\begin{array}{l}\text { Kriterien der } \\
\text { Urinausscheidung }\end{array}$ \\
\hline $\begin{array}{c}\text { Risk } \\
\text { (Risiko) }\end{array}$ & $\begin{array}{c}\text { erhöhtes Serumkreatinin um } \\
\text { Faktor 1,5 oder Abfall der } \\
\text { GFR um mehr als } 25 \%\end{array}$ & $\begin{array}{l}\text { Urinausscheidung unter } 0,5 \\
\mathrm{ml} / \mathrm{kg} / \mathrm{h} \text { für } 6 \mathrm{~h}\end{array}$ \\
\hline $\begin{array}{c}\text { Injury } \\
\text { (Schädigung) }\end{array}$ & $\begin{array}{c}\text { erhöhtes Serumkreatinin um } \\
\text { Faktor } 2 \text { oder Abfall der } \\
\text { GFR um mehr als 50\% }\end{array}$ & $\begin{array}{l}\text { Urinausscheidung unter } 0,5 \\
\mathrm{ml} / \mathrm{kg} / \mathrm{h} \text { für } 12 \mathrm{~h}\end{array}$ \\
\hline $\begin{array}{c}\text { Failure } \\
\text { (Versagen) }\end{array}$ & $\begin{array}{c}\text { erhöhtes Serumkreatinin um } \\
\text { Faktor } 3 \text { oder Abfall der } \\
\text { GFR um } 75 \% \text { oder } \\
\text { Serumkreatininwert von } 4 \\
\text { mg/dl }\end{array}$ & $\begin{array}{l}\text { Urinausscheidung unter } 0,3 \\
\mathrm{ml} / \mathrm{kg} / \mathrm{h} \text { für } 24 \mathrm{~h} \text { oder Anurie } \\
\text { für } 12 \mathrm{~h}\end{array}$ \\
\hline $\begin{array}{c}\text { Loss } \\
\text { (Verlust) }\end{array}$ & \multicolumn{2}{|c|}{$\begin{array}{l}\text { Persistierendes ANV = kompletter Verlust der } \\
\text { Nierenfunktion über } 4 \text { Wochen }\end{array}$} \\
\hline $\begin{array}{c}\text { ESKD = } \\
\text { End-Stage Kidney Disease } \\
\text { (Terminales Nierenversagen) }\end{array}$ & \multicolumn{2}{|c|}{ Terminales Nierenversagen über 3 Monate } \\
\hline
\end{tabular}

Tab.1: RIFLE-Kriterien 


\section{Inzidenz des ANV}

Die Bestimmung der Anzahl der Neuerkrankungen in einem bestimmten Zeitraum ist von einigen Schwierigkeiten begleitet, da in der Literatur angegebene Inzidenzen auf unterschiedlichen Definitionen des ANV und verschiedenen Patientenkollektiven basieren. Einige Studien wählen einen willkürlichen Kreatininwert (in der Literatur zwischen 130-600 $\mu \mathrm{mol} / \mathrm{l}$ ) aus, um das ANV zu definieren. Andere beziehen sich auf einen bestimmten Anstieg des Kreatinins im Verlauf oder die klinischen Schwere des ANV, welche eine Dialyseindikation darstellt. Dies ist einer der Gründe dafür, dass im Jahre 2004 eine internationale Konsensuskonferenz eine einheitliche Definition und Stadieneinteilung des ANV erarbeitete. Die unter 1.3.1.III. beschriebenen RIFLE-Kriterien stellen das Ergebnis dieser Bemühungen dar. Ein weiteres Problem bei der Feststellung der Inzidenzen des ANV ist die Unterscheidung zwischen dem akuten prärenalen Nierenversagen ohne manifeste Schädigung des Nierenparenchyms und der akuten ischämischen Tubulusnekrose, welche in den meisten Studien nicht gelingt (Lameire und Vanholder 1998). In Anlehnung an Hörl (2004) und Harrison et al. (2005) ist das ANV außerhalb der klinischen Versorgung im Krankenhaus selten. Bei hospitalisierten Patienten ist das ANV eine Komplikation, die bei ca. 4\% aller stationären Patienten auftritt. Im Rahmen eines Aufenthaltes auf der Intensivstation beträgt die Inzidenz zwischen 20-40\%. Die Inzidenz speziell im Bezug auf kardiochirurgische Operationen wird in der Literatur mit 0,7-31\% angegeben (Thakar et al. 2003). Auf diesen Aspekt wird in Punkt 1.4 genauer eingegangen.

\subsection{2 Ätiologie des ANV}

Das ANV kann als Komplikation bei vielen Erkrankungen auftreten, die aus diagnostischen und therapeutischen Überlegungen meist in drei Kategorien eingeteilt werden (Harrison et al. 2005).

\begin{tabular}{||c|c|l||}
\hline \multicolumn{1}{|c|}{ Art des Nierenversagens } & Häufigkeit & \multicolumn{1}{c||}{ Ursache } \\
\hline \hline Prärenales ANV bzw. prärenale Azotämie & $\begin{array}{c}\mathbf{( 5 5 \% -} \\
\mathbf{6 0 \%})\end{array}$ & $\begin{array}{l}\text { renale Hypoperfusion ohne } \\
\text { Beeinträchtigung des } \\
\text { Nierenparenchyms }\end{array}$ \\
\hline Eigentliches ANV bzw. renale Azotämie & $\mathbf{( 3 0 - 4 0 \% )}$ & $\begin{array}{l}\text { Schädigung des } \\
\text { Nierenparenchyms }\end{array}$ \\
\hline Postrenales ANV bzw. postrenale Azotämie & $\mathbf{( c a . 5 \% )}$ & $\begin{array}{l}\text { Zustände die eine } \\
\text { Harnwegsobstruktion bewirken }\end{array}$ \\
\hline
\end{tabular}

Tab.2: Art des Nierenversagens 


\section{Prärenales ANV}

Das prärenale ANV stellt die häufigste Variante des ANV dar (Joannidis et al. 1990). Wenn es im Rahmen eines Schockgeschehens, z.B. aufgrund von Blut- oder Flüssigkeitsverlusten, zu einer Zentralisation des Blutes kommt, wird die Durchblutung der Niere gedrosselt, um eine konstante Durchblutung von Herz und Gehirn gewährleisten zu können (Silbernagel 1989). Eine geringe Auswurfleistung des Herzens kann auch bei ausreichendem Blutvolumen zu einer verminderten Perfusion der Nieren führen. Dem prärenalen ANV liegt also eine Minderperfusion zugrunde, die einen Abfall der Nierendurchblutung und als Folge dessen einen Abfall der GFR nach sich zieht. Dieser Vorgang ist bis zu einem gewissem Maße sinnvoll und entspricht den unter Punkt 1.2 geschilderten physiologischen Anpassungsmechanismen der Niere auf sich ändernde Blutdruckverhältnisse. Deshalb ist auch in diesem Stadium eine volle Rekompensation der Nierenfunktion noch möglich. Zu diesem Zeitpunkt sind noch keine wesentlichen morphologischen Schädigungen der Niere festzustellen. Ist die Minderperfusion allerdings prolongiert oder unterschreitet der Blutdruck bestimmte Minimalwerte über ein bestimmtes Zeitintervall, dann kommt es zu ischämischen Tubuluszellnekrosen und es erfolgt der Übergang in ein ischämisches ANV (Joannidis 1990). In Anlehnung an Harrison et al. (2005) sind die wichtigsten dem prärenalen ANV zugrundeliegenden Ursachen folgende:

(1) Hypovolämie

a. Blutung, Verbrennung, Dehydrierung

b. Gastrointestinale Flüssigkeitsverluste: Erbrechen, Diarrhö, chir. Drainagen

c. Renale Flüssigkeitsverluste: Diuretika, osmotische Diurese (z.B. Diabetes mellitus), Nebenniereninsuffizienz

d. Sequestration von Flüssigkeit in den Extravasalraum: Pankreatitis, Peritionitis, Trauma, Verbrennungen, schwere Hypalbuminämie

(2) Vermindertes Herzzeitvolumen

a. Erkrankungen des Myokards, der Klappen, des Perikards; Arrhythmien; Tamponade

b. Andere: pulmonale Hypertonie, schwere Lungenembolie, Überdruckbeatmung

(3) Veränderter Quotient zwischen renalem und systemischem Gefäßwiderstand

a. Systemische Vasodilatation: Sepsis, Antihypertensiva, Nachlastsenker, Anästhesie, Anaphylaxie

b. Renale Vasokonstriktion: Hyperkalzämie, Noradrenalin, Adrenalin, Ciclosporin, Tacrolimus, Amphotericin B 
c. Zirrhose mit Aszites (hepatorenales Syndrom)

(4) Renale Minderperfusion mit Beeinträchtigung der renalen Autoregulation wie Cyclooxygenasehemmer und ACE-Hemmer.

(5) Hyperviskositätssyndrome: Multiples Myelom, Makroglobulinämie, Polyzythämie

\section{Renales / intrinsisches ANV}

Auf das renale und postrenale ANV wird an dieser Stelle weniger genau eingegangen als auf das prärenale, da sie für diese Arbeit nur eine untergeordnete Rolle spielen. Das renale ANV macht ca. 1/3 der Fälle aus und lässt sich nach pathologischen Kriterien in Erkrankungen der großen Nierengefäße, der Glomerula und des Nierengefäßsystems, tubulointerstitielle Erkrankungen und in die akute tubuläre Nekrose (ATN) einteilen (Joannidis 1990). Interessant ist, dass ca. 90\% der Fälle des renalen ANV der ATN zugeordnet werden müssen, welche aus einem prärenalen ANV resultieren können. In Anlehnung an Joannidis (1990) werden im Folgenden die häufigsten Ursachen des renalen ANV genannt:

(1) Tubuluszellschädigung

a. Ischämie

b. Medikamente/Toxine (Antibiotika, Zytostatika, Radiokontrastmittel)

c. Endogene Toxine (Myoglobin, Hämoglobin, Bence-Jones-Protein, Harnsäure)

(2) Akute tubulointerstitielle Erkrankungen

a. Allergische interstitielle Nephritis

b. Infektiöse Nephritis

c. Akute zelluläre Transplantatabstossung

(3) Glomeruläre Erkrankungen und Veränderungen der kleinen Nierengefäße

a. Akute Glomerulonephritis

b. Vaskulitis

c. Maligne Hypertonie, Prä-/Eklampsie

d. Sklerodermie

e. Röntgenkontrastmittel

f. Koagulopathien (Hämolytisch-urämisches Syndrom, Thrombotischthrombozytopenische Purpura)

(4) Erkrankungen der großen Nierengefäße

a. Nierenarterien (Thromboembolie, Cholesterinembolie, Vaskulitis)

b. Nierenvenenthrombosen 


\section{Postrenales ANV}

In ca. 5\% der Fälle kommt es zum postrenalen ANV, welches durch eine Abflussbehinderung meist im Bereich des Blasenhalses entsteht. Nach Harrison et al. (2005) unterscheidet man nach dem Ort der Verlegung:

(1) Nierenbecken (Steine, Fehlbildungen, Entzündungen)

(2) Ureter (Steine, Blutkoagel, Papillennekrosen, Tumoren, externe Kompression)

(3) Blasenhals (Neurogene Blase, Prostatahypertophie, Tumoren, Blutkoagel)

(4) Urethra (Striktur, angeborene Klappen, Phimose)

\subsubsection{Pathophysiologie des ANV}

Hinsichtlich der Pathophysiologie ist das prärenale ANV von der Initialphase des ischämischen ANV nicht zu unterscheiden, und der Übergang vom prärenalen ANV zur akuten tubulären Nekrose ist fließend. In dieser Phase führt ein vermindertes zirkulierendes Blutvolumen zu einer Aktivierung zentraler Barorezeptoren, welche direkt eine Steigerung des Sympathikotonus, eine Stimulation der Ausschüttung von Arginin-Vasopressin und indirekt eine Erhöhung von Angiotensin II bewirken. Dadurch soll über eine Vasokonstriktion eine Erhöhung des totalen peripheren Widerstands und damit des Blutdrucks erreicht werden. Noradrenalin wirkt aber auch direkt an der Niere und konstringiert über $\alpha$-Rezeptoren Nierengefäße und steigert die Reninausscheidung über $\beta$-Rezeptoren an der Makula densa. Angiotensin II, Noradrenalin und Arginin-Vasopressin als Folge einer systemischen Reaktion auf einen Blutdruckabfall bewirken also an der Niere eine Vasokonstriktion und eine Mesangiumzellkontraktion, in deren Folge der renale Blutfluss und die GFR abfällt. Wenn die Hypoperfusion ein bestimmtes Maß überschreitet oder zu lange andauert, können die renalen Autoregulationsmechanismen erschöpft werden und es kommt zu einer ischämischen Schädigung des Nierenparenchyms, welche weitere, die GFR senkende Konsequenzen, nach sich zieht. Im Zuge der ischämischen Zellschädigung kommt es zu einer endothelialen Dysfunktion, die ihrerseits die medulläre Ischämie weiter aufrechterhält. Außerdem führen Schäden von Tubulusepithelzellen zu tubulärer Obstruktion und einem backleak des Glomerulumfiltrats. Als Erklärung hierfür wird eine durch Hypoxie bedingte Zellschädigung diskutiert, durch die Tubuluszellen ihre Polarität verlieren und in der Folge - im Zuge der Redistribution der $\beta_{1}$-Integrine von der basolateralen auf die apikale Seite - von der 
Basalmembran abgelöst werden. Einerseits könnte das zur Verklumpung und Verlegung des Tubuluslumens, andererseits durch die entstandenen Poren zum Zurücklecken des Filtrats führen (Joannidis 1990). Auch diese Mechanismen würden den weiteren Abfall der GFR in der oligoanurischen Phase des ANV teilweise erklären. Als weiterer wichtiger Mechanismus, der nach bereits eingesetzter tubulärer Schädigung das Fortbestehen der reduzierten GFR verstärkt, gilt der tubuloglomeruläre Feedback, dessen physiologische Wirkungsweise unter Punkt 1.2.2 dargestellt wurde. Dieser physiologische Regulationsmechanismus, der eigentlich das Nephron vor Hyperfiltration schützen soll, führt in der Pathogenese des ANV zu einer weiteren Einschränkung des renalen Blutflusses und der GFR, weil die tubuläre Schädigung eine Herabsetzung der NaCl-Resorption und Wasser-Resorption bedingt und daher aufgrund erhöhter Flussraten und erhöhtem NaCl-Gehalt an der Makula densa den tubuloglomerulären Feedback auslöst. D.h., durch das Mesangium wird eine weitere Vasokonstriktion der Arteriolen und eine zusätzliche Verminderung der Filtrationsfläche vermittelt. Zusätzlich zu den oben genannten Mechanismen der tubulären Zellschädigung spielen Sauerstoffradikale und Lipidperoxydation eine wesentliche Rolle (Joannidis et al. 1989). Das Schädigungsmuster der Niere ist aufgrund ihrer speziellen anatomischen Architektur (siehe 2.2.1) sehr unterschiedlich. Besonders im inneren Nierenmark kommt es zu lokalen Zellnekrosen, da hier einerseits ein hoher Sauerstoffbedarf herrscht, aber andererseits aufgrund des Gegenstromprinzips eine reduzierte Sauersoffreserve besteht. Trotz dieser Heterogenitiät des Schädigungsmusters beim ANV kommt es fast immer zu einer generalisierten Antwort der Niere im Sinne eines Abfalls der GFR und Oligurie/Anurie (Joannidis 1990). In der Erholungsphase des ischämischen ANV ist eine Regeneration des Tubulusepithels, eine Erholung der Nierenperfusion und eine Wiederherstellung der GFR möglich (Harrison et al. 2005). Zusammenfassend lässt sich sagen, dass die akute tubuläre Nekrose als häufigste Form des ANV vor allem durch einen Abfall der GFR gekennzeichnet ist, welche durch folgende Faktoren verursacht bzw. verstärkt wird:

- Vasokonstriktion/Mesangiumzellkontraktion

- Tubuläre Obstruktion und backleak (=“Zurücklecken”)

- Tubuloglomerulärer Feedback (=“Rückkopplung”)

- Tubuläre Zellschädigung 


\subsubsection{Klinischer Verlauf des ANV}

Die oben geschilderten pathophysiologischen Vorgänge verlaufen bei einem prärenalen ANV, das nicht durch die Wiederherstellung einer adäquaten Nierendurchblutung oder die Behandlung der zugrundeliegenden Erkrankung beendet wird und in eine ischämische akute Tubulusnekrose und damit in ein renales ANV übergeht, klassischerweise in drei Phasen (Harrison et al. 2005).

\section{Initiale Schädigungsphase}

Diese erste Phase des ANV ist geprägt durch den Beginn der renalen Hypoperfusion und der Entwicklung einer ischämischen Schädigung. Sie dauert Stunden bis Tage. Der verminderte renale Blutfluss und der erniedrigte glomeruläre Ultrafiltrationsdruck, sowie die Behinderung des Flusses des Glomerulumfiltrates durch Zylinder aus nekrotischen Tubulusepithelien und der Mechanismus des Backleak führen zu einer abfallenden glomerulären Filtrationsrate. Die ischämische Schädigung betrifft vor allem medulläre Anteile des proximalen Tubulus $\left(\mathrm{S}_{3^{-}}\right.$ Segment und Pars recta) und die medullären Anteile der dicken aufsteigenden Henle-Schleife. Diese beiden Regionen haben einen hohen Bedarf an Sauerstoff, da hier energieverbrauchender ATP-abhängiger Teilchentransport stattfindet. Allerdings haben diese Bereiche selbst unter Normalbedingungen keine wesentliche Sauerstoffreserve, was in dem Prinzip der Harnkonzentrierung im Gegenstromprinzip und der damit zusammenhängenden besonderen Gefäßstruktur der Niere begründet ist. Die zelluläre Ischämie bewirkt einige Veränderungen im Metabolismus und der Funktionsfähigkeit der jeweiligen Zelle. Energieversorgung, Ionentransport und Membranintegrität werden kompromittiert und führen bei einem schweren Verlauf zu Nekrose oder Apoptose. Neuerdings scheint klar geworden zu sein, dass neben der Zellnekrose vor allem der durch Apoptose gesteuerte Zellumsatz eine wesentlichere Rolle in der Pathophysiologie der renalen Schädigung im Zuge eines ANV spielt (Hörl 2004). Die geschädigten Zellen schwellen an, ihr Zytoskelett wird zerstört, und sie verlieren ihre Polarität. Wichtig ist, dass in dieser Phase die Nierenschädigung durch Wiederherstellung des renalen Plasmaflusses begrenzt werden kann.

\section{Aufrechterhaltungsphase}

Die Dauer dieser Phase beträgt üblicherweise 1-3 Wochen wobei in seltenen Fällen auch Zeiträume von 1 bis zu 12 Monaten beobachtet wurden (Joannidis 1990). In der Aufrechterhaltungsphase hat sich ein Nierenzellschaden bereits etabliert, und die GFR hat sich an einem Tiefpunkt von typischerweise 5-10 ml/min stabilisiert. Es kommt hier zur minimalen Urinausscheidung und eventuell auch zu urämischen Komplikationen. Deshalb 
wird dieser Abschnitt im deutschsprachigen Raum auch oft Phase der Oligo-Anurie genannt. In der oligoanurischen Phase ist der Patient akut durch eine Überwässerung gefährdet, welche zu einem Lungen-oder Hirnödem führen kann. Des Weiteren ist besonders die Gefahr einer Hyperkaliämie zu beachten, welche zu schwerwiegenden Herzrhythmusstörungen führen kann. Sicherlich spielen vasoaktive Mediatoren wie vermindertes Stickstoffoxid, erhöhtes Endothelin-1, Adenosin oder plättchenaktivierender Faktor (PAF) eine große Rolle für den klinischen Verlauf des ANV (Harrison et al. 2005). Diese Mediatoren werden aus geschädigten Endothelzellen freigesetzt und fördern die anhaltende intrarenale und medulläre Ischämie. Des Weiteren spielt die Verstopfung der medullären Blutgefäße durch Sauerstoffradikale und andere Mediatoren ein Rolle. Ein regelrechter Teufelskreis wird durch den dauerstimulierten tubuloglomerulären Feedback ausgelöst.

\section{Genesungsphase}

Die Genesungsphase wird im deutschsprachigen Raum meist in eine polyurische Phase, welche Tage bis Wochen dauert und die Regenerationsphase, welche 1-3, maximal aber 12 Monate dauert, unterteilt. Diese beiden Phasen werden an dieser Stelle in Anlehnung an Harrison et al. (2005) gemeinsam betrachtet. In der Genesungsphase findet eine Wiederherstellung und Regenerierung der Nierenparenchymzellen, insbesondere der Tubulusepithelzellen, statt, welche in die weitgehende Normalisierung der Nierenfunktion mündet. Wenn bei der konsequenten Steigerung der GFR die Wiederherstellung der Epithelzellfunktion verzögert ist, droht eine massiv verstärkte Diurese. In der polyurischen Phase kommt es zu einer vermehrten Ausscheidung von zurückgehaltenen Elektrolyten, Wasser und anderen Substanzen. Oft wird diese Situation noch durch eine begleitende Diuretikatherapie gefördert. In der polyurischen Phase droht dem Patienten ein Elektrolytverlust, der wiederum eine eingeschränkte Nierenfunktion nach sich ziehen kann. Deshalb sind in dieser Phase eine adäquate Flüssigkeits- und Elektrolytbilanzierung essentiell.

\subsubsection{Diagnose des ANV}

Die Diagnose eines ANV basiert neben Anamneseerhebung und Klinik im Wesentlichen auf der Erfassung der Diuresemenge und Flüssigkeitsbilanz des Patienten und laborchemischen Untersuchungen von Serum und Urin. Bei Verdacht auf ein postrenales ANV können radiologische Untersuchungen wie Sonographie, Computer- oder Mangnetresonanztomographie notwendig werden. Zur Erfassung vaskulärer Obstruktionen kann die Doppler-Sonographie und Magnetresonanztomographie beitragen. Eine Nierenbiopsie kann nach Ausschluss eines prä- oder postrenalen ANV, wenn 
Symptomatologie, Urinanalyse und Laborwerte eher gegen ein ischämisches oder nephotoxisches ANV sprechen, sinnvolle Hinweise zur Aufdeckung von beispielsweise Glomerulonephritiden oder Vaskulitiden liefern, die auf eine spezifische Therapie ansprechen würden (Harrison et al. 2005). Prinzipiell kann zwischen einem akuten und chronischen Nierenversagen unterschieden werden, wobei ein kurzfristiger Anstieg von Harnstoff und Kreatinin im Serum eher für ein akutes Geschehen sprechen und Anämie, Neuropathie, radiologische Zeichen einer renalen Osteodystrophie und kleine, vernarbte Nieren eher einen chronischen Verlauf andeuten.

\section{Klinik des pärenalen ANV}

Klinische Anzeichen für ein prärenales ANV sind Symptome eines erniedrigten effektiven Blutvolumens bzw. Flüssigkeitsmangels, wie:

- Durst, orthostatischer Schwindel

- Orthostatische Hypotonie und Tachykardie

- Verminderter Hautturgor, stehende Hautfalten, trockene Zunge

- Trockene Schleimhäute, vermindertes Schwitzen in den Achseln

$\mathrm{Zu}$ beachten sind insbesondere eine eventuelle Abnahme der Urinausscheidung und die Überprüfung der Medikamentenanamnese im Sinne einer neu begonnenen Therapie mit Cylooxygenasehemmern, ACE-Hemmern und Angiotensin-II-Rezeptorblockern. Weitere Zustände, die zu einem erniedrigten effektiven zirkulierenden Blutvolumen führen wie z.B. fortgeschrittene Herzinsuffizienz oder Leberversagen, sollten bei der klinischen Untersuchung bedacht werden. Grundsätzlich sollten Zustände, die eine längere schwerwiegende Minderperfusion der Niere bedingen können wie z.B. eine Hypovolämie, ein septischer Schock oder ausgedehnte Operationen wie z.B. Herzchirurgie, den Verdacht auf die mögliche Entstehung eines ischämischen renalen ANV lenken. Wenn zudem nach Normalisierung der systemischen Hämodynamik durch iatrogene Maßnahmen die Zeichen eines Nierenversagens bestehen bleiben, wird ein ischämisches ANV wahrscheinlicher. In Abgrenzung zum nephrotoxischen ANV ist es wichtig, nephrotoxische Medikamente sowie Röntgenkontrastmittelexposition in die pathopyhsiologischen Überlegungen einzubeziehen. 
Auch endogene Toxine wie Myoglobin, Hämoglobin, Harnsäure, Myelomproteine und erhöhte Serumkalziumspiegel können eine pathopysiologische Bedeutung haben. Obwohl das ischämisch und nephrotoxisch bedingte ANV mehr als 90\% ausmacht (Harrison et al. 2005), gibt es weitere Differentialdiagnosen der Oligurie. In Anlehnung an den Punkt 1.2.3 sind hier insbesondere zu nennen:

- Nierenarterienverschluss/Nierenvenenthrombose

- Schwere Glomerulonephritis/Vaskulitiden

- Interstitielle Nephritis/Pyelonephritis

- Hepatorenales Syndrom

- Disseminierte intravasale Gerinnung

- Hypertensive Krise

- Obstruktionen in allen Abschnitten der ableitenden Harnwege

\section{Labor}

Wenn infolge einer eingeschränkten Nierenexkretionsfunktion stickstoffhaltige harnpflichtige Substanzen vermehrt im Serum ansteigen, nennt man diesen Zustand Azotämie (Krautzig 2004). Bei Progredienz dieser Entwicklung kann 5-10 Tage nach einem akuten Nierenversagen eine Urämie entstehen, ein klinisches Syndrom, das neben der pathologischen Erhöhung von Harnstoff, Kreatinin, Harnsäure, Kalium, Magnesium, Phosphat und Urämiegiften wie Guanidinderivaten und Phenolen diverse Symptome im Gastrointestinaltrakt, dem hämatologischen, kardiovaskulären und Nervensystem bis hin zum Koma verursachen kann (Krautzig 2004). Als klinische Marker für eine Retention werden Kreatinin und Harnstoff, zwei selbst ungiftige Substanzen, im Serum bestimmt. Kreatinin ist ein Abbauprodukt des Muskelstoffwechsels und wird renal in erster Linie glomerulär filtriert, in geringerem Maß aber auch tubulär sezerniert. Die Normalwerte betragen für Frauen $<0.9$

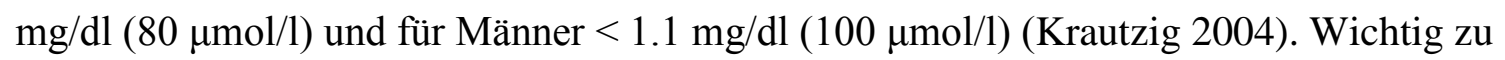
beachten ist, dass Kreatinin im Serum erst ab einer Einschränkung der Nierenfunktion von mehr als 50-60\% (Krautzig 2004) erfolgt und es deshalb eine diagnostische Lücke in Bereichen nur leicht eingeschränkter GFR gibt. Außerdem muss noch in Betracht gezogen werden, dass falsch-hohe Werte je nach Labormethoden durch Pseudokreatinine wie z.B. 
Ketonkörper oder auch Muskelläsionen, extremer muskulärer Belastung oder sehr hoher Muskelmasse per se auftreten können. Falsch-niedrige Werte können bei geringer Muskelmasse z.B. im Zustand der Kachexie oder bei einer Hyperbilirubinämie vorkommen (Krautzig 2004). Harnstoff als Endprodukt des Proteinstoffwechsels der Leber wird analog zur produzierten Harnmenge ausgeschieden und hat einen Normalwertbereich von 10-50 mg/dl (2-8 mmol/l) (Krautzig 2004). Die Konzentration im Serum ist aber auch von der Proteinzufuhr und Zuständen mit erhöhtem Katabolismus wie z.B. Fieber oder Sepsis abhängig. Bei Nierenfunktionseinschränkung allerdings erhöht sich der Wert erst bei einer Einschränkung von $\geq 60-70 \%$ (Krautzig 2004). D.h., bei beginnender latenter Niereninsuffizienz wie dem ANV ist Kreatinin im Serum der bessere Marker. Deshalb wurde er in dieser Studie verwendet. In fortgeschrittenen Stadien allerdings korreliert der Harnstoffwert im Serum besser mit dem klinischen Schweregrad der Nierenfunktionseinschränkung. Um ein noch genaueres Maß für die Abschätzung der GFR zu erhalten, kann durch Bestimmung des Kreatiningehalts im 24-Stunden-Sammelurin die Kreatininclearance errechnet werden. Allgemein beschreibt die clearance ( = "Klärrate") eines Stoffes in der Dimension $\mathrm{ml} / \mathrm{min}$ das Plasmavolumen pro Zeiteinheit, in welcher dieser renal ausgeschiedene Stoff gelöst war. Am Beispiel des Kreatinins berechnet sich die Clearance [Cl- Krea (ml/Min)] aus der Konzentration des Kreatinins im Urin [U$\mathrm{Krea}(\mathrm{mg} / \mathrm{dl})$ ], dividiert durch die Konzentration der Substanz im Plasma [S-Krea(mg/dl)] und multipliziert mit dem Urinzeitvolumen [V (ml) / t (Min)] .

$$
\mathrm{Cl}-\mathrm{Krea}(\mathrm{ml} / \mathrm{Min})=\frac{\mathrm{U}-\mathrm{Krea}(\mathrm{mg} / \mathrm{dl}) \times \mathrm{V}(\mathrm{ml})}{\mathrm{S}-\mathrm{Krea}(\mathrm{mg} / \mathrm{dl}) \times \mathrm{t}(\mathrm{Min})}
$$

Formel 1: Kreatininclearance

Die endogene Kreatininclearance entspricht annähernd der GFR und sollte normal etwa 120 $\mathrm{ml} / \mathrm{min}$ betragen. Sie ist eine sinnvolle diagnostische Maßnahme für das frühzeitige Erkennen einer Nierenfunktionseinschränkung v.a. auch dann, wenn das Serumkreatinin noch im Normbereich liegt (Krautzig 2004). Wenn kein Sammelurin vorliegt, kann die Kreatininclearance $\left(\mathrm{Cl}_{\text {Krea }}\right)$ in $\mathrm{ml} /$ min auch näherungsweise mit der Formel nach Cockcroft und Goult abgeschätzt werden. Sie berechnet sich aus dem Produkt von 150 minus Lebensalter (LA) und dem Körpergewicht in Kilogramm (KG), dividiert durch das 
Serumkreatinin in $\mu$ mol/l minus 10\% für Männer bzw. 15\% für Frauen (a) (Krautzig 2004). Siehe dazu Formel 2.

$$
\mathrm{Cl}_{\mathrm{Krea}}=[(150-\mathrm{LA}) \cdot \mathrm{KG} / \text { Serumkreatinin }]-\mathrm{a}
$$

Formel 2: Cockcroft und Goult

\section{Urinuntersuchung, Sediment, Urinindizes}

Die Untersuchung des Urins war nicht Bestandteil unserer Untersuchungen und wird daher an dieser Stelle nur knapp und in Bezug auf das prärenale ANV abgehandelt.

Neben der Erhöhung der Retentionsparameter ist das Versiegen der Harnsekretion mit OligoAnurie in $82 \%$ der Fälle (Harrison et al. 2005) das wichtigste Leitsymptom des ANV. Die restlichen Fälle verlaufen normo- oder polyurisch. Von einer Oligurie spricht man ab einer Urinproduktion unter $500 \mathrm{ml}$ pro Tag und von einer Anurie bei einer Urinproduktion unter $100 \mathrm{ml}$ pro Tag. Anurische Verläufe sind bei kompletter Obstruktion oder komplizierten Verläufen von prärenalen oder renalen ANV denkbar. Eine partielle Obstruktion kann aufgrund gestörter Konzentrierungsmechanismen des Harns zu Polyurie führen. Als Polyurie bezeichnet man ein Harnvolumen von mehr als $2000 \mathrm{ml}$ pro 24 Stunden (Krautzig 2004). Die Erfassung der Diureseleistung, also die tägliche Bilanzierung von Flüssigkeitseinfuhr und Ausfuhr sowie des Körpergewichts, ist neben der Kontrolle von Elektrolyten und Retentionsparametern deshalbvon entscheidender Bedeutung.

Im Urinsediment lassen sich ebenfalls Hinweise für die Ätiologie eines ANV finden. Durchsichtige hyaline Zylinder, welche aus normalen Urinbestandteilen bestehen, sind typisch für ein prärenales ANV(Harrison et al. 2005). Sie bestehen hauptsächlich aus TammHorsfall-Protein, einem Protein, welches aus den Epithelzellen im Bereich der Henleschen Schleife sezerniert wird. Außerdem typisch für ein prärenales Nierenversagen sind im konzentrierten Urin entstehende, einzelne Harnsäurekristalle mit pleomorphen Formen Die klinisch wichtigste Differentialdiagnose beim ANV ist die Abgrenzung vom prärenalen ANV zur akuten Tubulusnekrose (ATN) bzw. dem ischämischen ANV. Die biochemische Analyse von Plasma und Urin kann bei dieser Unterscheidung sehr hilfreich sein, denn der wesentliche pathophysiologische Unterschied zwischen Patienten mit prärenalen und ischämischen ANV mit ATN besteht darin, dass Erstere aufgrund des intakten Tubulussystems noch die Fähigkeit besitzen, massiv Natrium und somit auch Wasser 
rückzuresorbieren, um das intravasale Volumen wieder zu erhöhen, und dass die Anderen aufgrund des bereits eingetretenen Tubulusschadens diese Fähigkeit teilweise oder komplett eingebüßt haben. Dies macht sich natürlich bei der Natriumkonzentration im Urin, der Urinosmolalität und dem spezifischen Gewicht des Urins bemerkbar. In Anlehnung an Harrison et al. (2005) sind diese Zusammenhänge in Tab. 1 dargestellt.

\begin{tabular}{|c||c|c|}
\hline $\begin{array}{c}\text { Diagnostischer } \\
\text { Index }\end{array}$ & Prärenales ANV & Ischämisches ANV \\
\hline Urinnatriumkonzerntration $(\mathrm{mmol} / \mathrm{l})$ & $<10$ & $>20$ \\
\hline Urinosmolalität $\left(\mathrm{mosmol} / \mathrm{kg} \mathrm{H}_{2} \mathrm{O}\right)$ & $>500$ & $\sim 300$ \\
\hline Spezifisches Gewicht des Urins $(\mathrm{g} / \mathrm{l})$ & $>1.020$ & $\sim 1.010$ \\
\hline
\end{tabular}

Tab.3: Urinindizes

Ein noch sensitiverer Indikator in der Differenzierung von prärenalem und ischämischem ANV ist die Fraktionelle Natriumausscheidung $\left(\mathrm{FE}_{\mathrm{Na}}\right)$ in Prozent. Die $\mathrm{FE}_{\mathrm{Na}}$ stellt eine Beziehung zwischen Natrium und Kreatininclearance her. Sie basiert ebenfalls auf dem oben dargestellten Sachverhalt der unterschiedlichen Rückresorptionskapazitäten bei prärenalem und ischämischem ANV. Die Fraktionelle Natriumausscheidung berechnet sich aus dem Quotienten des Produktes aus Urin-Natriumkonzentration $\left(\mathrm{U}_{\mathrm{Na}}\right)$ und PlasmaKreatininkonzentration $\left(\mathrm{P}_{\mathrm{Cr}}\right)$ und dem Produkt aus Plasma-Natriumkonzentration $\left(\mathrm{P}_{\mathrm{Na}}\right)$ und Urin-Kreatininkonzentration $\left(\mathrm{U}_{\mathrm{Cr}}\right)$ multipliziert mit 100 in Prozent.

$\left(\mathrm{FE}_{\mathrm{Na}}\right)(\%)=\left(\mathrm{U}_{\mathrm{Na}} \cdot \mathrm{P}_{\mathrm{Cr}} / \mathrm{P}_{\mathrm{Na}} \cdot \mathrm{U}_{\mathrm{Cr}}\right) \cdot 100$

Formel 3: Fraktionelle Natriumausscheidung

Ein Wert unter $1 \%$ spricht für ein prärenales ANV, ein Wert über 1\% spricht für ein ischämisches renales ANV. Siehe hierzu Tab. 4 in Anlehnung an Harrison et al. (2005). 


\begin{tabular}{|c|c|c|}
\hline Fraktionelle Natriumausscheidung( $\left.\mathrm{FE}_{\mathrm{Na}}\right)$ & Prärenales ANV & Ischämisches ANV \\
\hline \hline$\left(\mathrm{U}_{\mathrm{Na}} \cdot \mathrm{P}_{\mathrm{Cr}} / \mathrm{P}_{\mathrm{Na}} \cdot \mathrm{U}_{\mathrm{Cr}}\right) \cdot 100(\%)$ & $<1 \%$ & $>1 \%$ \\
\hline
\end{tabular}

Tab.4: Fraktionelle Natriumausscheidung

\subsubsection{Komplikationen des ANV}

Das ANV bringt aufgrund seiner Pathophysiologie und der Beeinträchtigung des Elektrolytund Wasserhaushaltes häufig schwere Komplikationen mit sich. Die verminderte Salz- und Wasserausscheidung der Niere führt zu einem intravasalen Flüssigkeitsüberschuss, der sich in der Folge auch auf die anderen Kompartimente, v.a. den Extrazellulärraum ausweitet, was sich klinisch als Ödeme manifestiert. Neben einer metabolischen Azidose finden sich häufig auch eine Hyponatriämie, Hyperkaliämie, Hypokalzämie und Hypermagnesiämie. Eine leichte Hyperphosphatämie, eine leichte Anämie und eine verlängerte Blutungszeit können ebenfalls vorliegen. Die eingeschränkte Ausscheidung stickstoffhaltiger harnpflichtiger Substanzen führt über eine Azotämie zur Urämiesymptomatik. Neben den unten aufgeführten Komplikationen sind noch leichte gastrointestinale Blutungen zu nennen, welche in 10-30\% auftreten (Harrison et al. 2005).

\section{Erhöhung des Extrazellurärvolumens}

Durch die verminderte Ausscheidung von Natrium und konsekutiv $\mathrm{H}_{2} \mathrm{O}$ wird der Organismus überwässert. In leichter Ausprägung lässt sich das an einer Gewichtszunahme, einer erhöhten Jugularvenenfüllung bzw. einem erhöhten ZVD (zentralvenöser Druck), lageabhängigen Ödemen oder basal feuchten Rasselgeräuschen bei der Auskultation der Lunge erkennen. In seiner weiteren Ausprägung ist der Übergang zum Lungenödem fließend und gerade bei Patienten, die intravenös Flüssigkeit oder Nahrung appliziert bekommen, kann die Hypervolämie sehr problematisch werden. Die übermäßige Verabreichung von freiem Wasser kann zu Hypoosmolalität und Hyponatriämie führen, welche ein Hirnödem mit neurologischen Symptomen bis hin zum Krampfanfall oder Koma nach sich ziehen kann.

\section{Hyperkaliämie}

Bei oligurischen oder anurischen Patienten steigt das Serumkalium täglich um $0.5 \mathrm{mmol} / \mathrm{l}$ (Harrison et al. 2005). Die wichtigsten dazu beitragenden Umstände sind die gestörte Ausscheidung in der Niere, die Freisetzung von intrazellulärem Kalium aus geschädigtem 
Gewebe und die meist bestehende metabolische Azidose, die im Austausch mit Wasserstoffionen einen Ausstrom von Kalium aus den Zellen zur Folge hat. Bei einer Hyperkaliämie fallen im EKG eine spitze hohe T-Welle, der Verlust der P-Welle und eine Verbreiterung des gesamten QRS-Komplexes (insbesondere Rechtsschenkelblock) auf und es kann zu Bradykardien kommen. Bei Patienten mit Rhabdomyolyse, Tumorlysesyndrom oder starker Hämolyse kann die Hyperkaliämie schon zumDiagnosezeitpunkt sehr stark ausgeprägt sein.

\section{Metabolische Azidose}

Der Metabolismus von Proteinen hat pro Tag etwa 50-100 mmol gebundene, nichtflüssige Säuren zur Folge (Harrison et al. 2005), welche normalerweise über die Niere ausgeschieden werden. Ist deren Funktion eingeschränkt, häufen sich diese Moleküle im Serum an und verändern den Säure-Base-Haushalt. Da das fein eingstellte Verhältnis von Säuren und Basen in den Kompartimenten durch Akkumulation zu Gunsten der Säuren verschoben wird, kommt es bei zunehmender Insuffizienz der Puffersysteme zur metabolischen Azidose. Besonders stark ausgeprägt ist die Gefährdung für eine metabolische Azidose bei zusätzlicher endogener Produktion von sauren Metaboliten wie bei einer Ketoazidose im Rahmen von Diabetes mellitus oder Fasten, einer Laktatazidose bei genereller Gewebehypoperfusion, Lebererkrankungen, Sepsis oder dem vermehrten Abbau von Ethylenglykol oder Methanol.

\section{Infektionen}

Infektionen im Rahmen eines ANV treten zu etwa 50-90\% auf und stellen mit dem durch sie verursachten Anteil von $75 \%$ an Todesfällen auch eine sehr schwerwiegende Komplikation dar (Harrison et al. 2005). Inwieweit Patienten mit ANV eine Immunabwehrdefizienz haben oder die hohe Rate an Infektionen durch iatrogene Maßnahmen bedingtist, ist unklar.

\section{Urämisches Syndrom}

Das urämische Syndrom ist ein klinisches Syndrom, welches sämtliche durch eine fortgschrittene Nierenisuffizienz verursachten Symptome umfasst. Diese Symptome sind sehr vielfältig und betreffen dermatologische, gastrointestinale, hämatologische, kardiovaskuläre, neuromuskuläre und endokrine Organsysteme. Einige besonders schwerwiegende Auswirkungen sollen hier nochmals hervorgehoben werden:

- Flüssigkeitsretention bis hin zum Lungenödem

- Pleuritis und/oder Perikarditis mit Ergussbildung 
- Herzrhythmusstörungen bis hin zum Kammerflimmern

- Enzephalopathie bis hin zum Koma

\subsubsection{Therapiemöglichkeiten des ANV}

\section{Prävention}

Durch engmaschige und strikte Kontrolle der kardiovaskulären Parameter und des Intravasalvolumens sollte einem beginnenden Flüssigkeitsverlust bzw.

Flüssigkeitsbewegungen in extravasale Kompartimente mit Volumensubstitution begegnet werden, um einem ANV vorzubeugen. Dies gilt insbesondere für ältere Patienten, Patienten mit Diabetes mellitus und solchen mit bereits vorbestehendem Nierenversagen. Die Inzidenz eines ischämischen ANV nach großen Operationen, bei denen Flüssigkeitsverluste bzw. Verschiebungen in andere Kompartimente - gepaart mit hämodynamischen Faktoren- die Niere belasten, kann durch eine zügige und aggressive Wiederherstellung des Intravasalvolumens deutlich gesenkt werden. Allgemein lässt sich sagen, dass eine optimierte Hämodynamik nicht nur durch ein ausreichendes Intravasalvolumen, sondern auch durch suffiziente periphere Widerstände und ausreichende Inotropie am Herzen bei der Entstehung eines ANV präventiv wirken (Harrison et al. 2005). Zudem ist es von Bedeutung, einer sich entwickelnden Azidose frühzeitig entgegenzuwirken. Um einer Pigmentnephropathie im Sinne einer Verlegung der Nierentubuli mit Hämoglobin entgegenzuwirken, ist es wichtig, einen Abfall des Hämoglobins zu monitoren und diesen so gering wie möglich zu halten. Dabei spielen nicht nur die Blutverluste eine Rolle, sondern z. B. auch die Hämolyse durch die extrakorporale Zirkulation. Eine grundsätzliche Überlegung sollte sein, dass das Serumkreatinin v. a. bei jüngeren oder älteren Patienten ein oft relativ unempfindlicher Parameter ist und die GFR oft überschätzt wird. Deshalb empfiehlt es sich, zur Einbeziehung von Größe und Körpergewicht die Näherungsformel nach Cockcroft-Gault $\left(\mathrm{Cl}_{\mathrm{Krea}}=[(150-\right.$ LA) · KG / Serumkreatinin] - a) anzuwenden, um die GFR realistischer einschätzen zu können (Harrison et al. 2005). Die Indikation für die Gabe von Diuretika, Cyclooxygenasehemmern, ACE-Hemmern oder Angiotensin-II-Rezeptorhemmern ist bei Patienten mit Verdacht auf Hypovolämie streng zu stellen (Harrison et al. 2005). 


\section{Spezifische konservative Therapie}

Beim prärenalen hypovolämischen ANV steht die Flüssigkeitssubstitution, welche sich in der Art der Zusammensetzung an der Zusammensetzung des verlorengegangenen Volumens orientieren sollte, im Vordergrund. Bei einer Hypovolämie, die durch eine schwere Blutung verursacht ist, werden Erythrozytenkonzentrate und Elektrolytlösung bzw. Plasmaexpander transfundiert. Bei Plasmaverlusten, wie sie beispielsweise bei Verbrennungen oder Pankreatitis auftreten, sollte mit isotoner Kochsalzlösung substituiert werden. Erhöhte Verluste von Flüssigkeit über den Harn oder den Gastrointestinaltrakt werden meist mit hypotoner Lösung, z.B. $0.45 \% \mathrm{NaCl}$ behandelt, da die Verluste meist auch hypotonen Charakter haben. Die Therapie verläuft unter ständiger Kontrolle des Serumkaliums und der Säure-Base-Parameter, so dass, wenn erforderlich, Kalium oder Bicarbonat substituiert werden können (Harrison et al. 2005).

Bei vermindertem Herzzeitvolumen z.B. im Rahmen von Herzinsuffizienz kann es notwendig werden, positiv inotrope Substanzen, Medikamente zur Vor- und Nachlastsenkung oder mechanische Unterstützungssysteme wie beispielsweise eine intraaortale Ballonpumpe einzusetzen.

Es gibt eine Reihe von Substanzen, die meist vasodilatatorisch wirken und von denen man sich eine Verbesserung der Durchblutung der Niere zur Unterbrechung der im Teil Pathophysiologie beschriebenen Mechanismen erhofft. In tierexperimentellen Modellen sind diese teils erfolgversprechend, beim Menschen allerdings bisher ohne nachgewiesenen regelmäßigen Nutzen oder Effizienz (Harrison et al. 2005). Beispiele hierfür sind:

- Artriales natriuretisches Peptid (ANP)

- Niedrig dosiertes Dopamin

- Endothelin- Antagonisten

- Schleifendiuretika

- Kalziumkanalblocker

- Prostaglandinanaloga

- Antioxidantien

Ein relativ erfolgversprechendes Forschungsgebiet stellt auch die Anwendung von Wachstumsfaktoren wie IGF-1, EGF oder HGF dar. Von ihnen erhofft man sich eine 
Beschleunigung der Regeneration des Tubulusepithels (Joannidis et al. 1994). Zu den Diuretika, in erster Linie Schleifendiuretika, Thiazide oder diuretisch wirkende Stoffe wie Mannit ist zu sagen, dass sie einer strengen Indikationsstellung bedürfen, da sie keinen nachgewiesenen positiven Einfluss auf die Entwicklung oder Ausbildung eines ANV haben (Harrison et al. 2005). Sie sollten nur eingesetzt werden, wenn eine massive intravasale Volumenerhöhung vorliegt und von einer durch ihre Gabe bessere Beherrschung klinischer Probleme auszugehen ist. Von Dopamin erhoffte man sich bei niedrigdosierter Gabe unterhalb der Dosis, die die pressorische Wirkung entfaltet, eine bessere Nierendurchblutung, eine erhöhte GFR und eine Hemmung der tubulären Rückresorption mit der Folge einer besseren Ausscheidung. Allerdings ist das heute obsolet, denn zahlreiche Studien haben belegt, dass die kardialen Probleme und zahlreichen Nebenwirkungen bei der Therapie im Vordergrund stehen und durch die Dopamingabe kein prophylaktischer oder therapeutischer Nutzen entsteht (Harrison et al. 2005). Einer eventuellen Hyperkaliämie sollte mit einer Zufuhrbeschränkung von Kaliumzusätzen, kaliumsparenden Diuretika, kaliumbindenden Ionenausstauschharzen, Glucose plus Insulin, Natriumbicarbonat oder Kalziumglukonat begegnet werden. Ist diese Maßnahme nicht ausreichend, so muss eine Hämodialyse durchgeführt werden. Einer metabolischen Azidose kann mit Einschränkung der Proteinzufuhr, Natriumbicarbonat oder im fortgeschrittenen Fall ebenfalls mit Dialyse begegnet werden.

\section{Nierenersatzverfahren}

a) Indikation

Führen die oben genannten konservativen Therapien nicht zu einer Erhöhung der Diurese oder Stabilisierung der Hämodynamik, muss eine Nierenersatztherapie begonnen werden. Strenge einheitliche Indikations-Kriterien für deren Einsatz gibt es nicht. Trotzdem bestehen allgemein akzeptierte Indikationen zur Einleitung einer Nierenersatztherapie bei ANV wie persistierende Hyperkaliämie, eine schwere metabolische Azidose, diuretikaresistente Hyperhydratation oder das Vorliegen urämischer Symptome (Hörl 2004). Laut des Notfallmanuals des Zentrums für Innere Medizin der Universitätsmedizin Göttingen sind die einzelnen Indikationen zur Hämodialyse ein Kalium im Serum > 6,5 mmol/1, Lungenödem, Urämie-Symptome ( Gastritis, Perikarditis, Enzephalopathie) oder eine metabolische Azidose mit einem $\mathrm{ph}<7,0$. Bei der Entwicklung einer progredienten Azotämie wird ein prophylaktischer Dialysebeginn vor Auftreten von Symptomen befürwortet (Hörl 2004). 
b) Übersicht

Von dem technischen Standpunkt aus betrachtet gibt es eine ganze Reihe von Verfahren, die auf teils unterschiedlichen chemisch-physikalischen Mechanismen beruhen und nach unterschiedlichen Kriterien klassifiziert werden können. Die wichtigsten Verfahren sind die Hämodialyse, die Hämofiltration, die Hämodiafiltration und die Peritonealdialyse. Mit Ausnahme der Peritonealdialyse wird bei allen diesen Verfahren das Blut außerhalb des Körpers über künstliche Membranen gereinigt. Eine weitere Einteilung der Verfahren kann jeweils nach der Art der verwendeten Membranen, der intermittierenden oder kontinuierlichen Anwendung, der Art des Antriebs und der gewählten Gefäßzugänge stattfinden (Hörl 2004).

c) Membranen

Über die bei der Dialyse benutzten Cellulosemembranen findet in erster Linie Diffusion statt, was bewirkt, dass v.A. kleinere Moleküle effektiv eliminiert werden, wobei größere Moleküle die Membran unter Umständen nicht passieren können. Sogenannte High-Flux-Membranen kommen v.A. bei der Hämofiltration zum Einsatz und besitzen eine hohe hydraulische Permeabilität, die auf Konvektion beruht und eine effektive Elimination von größermolekularen Stoffen erlaubt. Ein weiterer Vorteil gegenüber den Cellulosemembranen ist ihre bessere Biokompatibilität.

d) Gefäßzugänge

Bei den Gefäßzugängen gibt es grundsätzlich die Möglichkeit ein große Vene mit einem Doppellumenkatheter für die venovenöse Technik oder eine große Vene und eine Arterie für die arteriovenöse Technik zu punktieren. Längerfristig kann ein arteriovenöser Shunt angelegt werden. Bei den arteriovenösen Formen wird der Blutfluss durch den Gradienten zwischen mittlerem arteriellem Blutdruck und zentralvenösem Druck sichergestellt. Bei venovenösen Formen muss diese Leistung von einer Pumpe übernommen werden. Die arteriovenösen Zugänge werden aber heute nur noch in Ausnahmefällen bei therapieresistenter Überwässerung oder Elektrolytentgleisungen bei extrem eingeschränkter Nierenfunktion angewandt (Hörl 2004).

e) Dialyse vs. Filtration

Der grundlegende Unterschied zwischen dem Prinzip der Dialyse und der Filtration ist die Art des chemisch-physikalischen Stoffaustausches. Bei der Dialyse findet an der Membran eine Diffusion gemäß der Brownschen Molekularbewegung statt und die Moleküle verteilen sich 
in Abhängigkeit ihrer Konzentrationsgradienten. Bei der Hämofiltration findet der Stoffaustausch durch Strömung statt. Diesen Vorgang nennt man Konvektion und er ähnelt der Filtration im Glomerulus bei der auch Stoffe entlang eines hydrostatischen Gradienten eine permeable Membran passieren. Was in der Niere dann als Reabsorbtion der benötigten Elektrolyte und Wasser im Tubulussystem erfolgt, muss bei der Hämofiltration durch substituierte Elektrolytlösungen von Außen erfolgen. Die Folge dieser unterschiedlichen Mechanismen ist eine bessere Clearance von mittlemolekularen Stoffen bei Hämofiltration und von kleinmolekularen Stoffen bei der Dialyse. Die Hämodiafiltration vereint die beiden Prinzipien von Diffusion und Konvektion.

f) intermittierend vs. kontinuierlich

Die Unterscheidung zwischen intermittierenden und kontinuierlichen Verfahren basiert auf der Tatsache, dass die intermittierende Hämodialyse nur einmal täglich bzw. jeden zweiten Tag mit einer Dauer von ca. 4 Stunden durchgeführt wird und die kontinuierlichen Verfahren, zu denen die langsame kontinuierliche Ultrafiltration, die kontinuierliche Hämofiltration und Dialyse und die kontinuierliche Hämodiafiltration gehört, über 24 Stunden oder sogar Tage bis Wochen durchgeführt wird. Es liegen beim Vergleich von kontinuierlichen Verfahren zu intermittierenden einige Vorteile auf der Hand, wie die Erzielung konstanter Spiegel in der Zusammensetzung von Körperflüssigkeiten steady state. Schnelle Volumenschwankungen und Veränderungen in der Zusammensetzung und Osmolalität der Extrazellulärflüssigkeit bleiben somit aus. Diese metabolischen und hämodynamischen Vorteile schlagen sich aber nicht in einer eindeutigen Datenlage nieder. In einigen Studien wird der Vorzug der kontinuierlichen Nierenersatztherapien postuliert (Ronco (1999), Bellomo (1999)). Aber letztendlich wird deren Aussage relativiert und kein signifikanter Unterschied bezüglich der Erholung vom ANV oder dem Überleben zwischen intermittierenden und kontinuierlichen Methoden als bewiesen angesehen (Hörl 2004).

Des Weiteren gibt es noch langsam kontinuierliche Verfahren wie slow efficient daily dialysis (SLEDD) oder extenteded daily dialysis (EDD) die beachtenswerte Methoden für die Zukunft darstellen könnten (Hörl 2004). Diese Verfahren werden auch Hybridverfahren genannt. 
Um einen Überblick über die der Literatur am meisten verwendeten Begriffe bezüglich der Nierenersatzverfahren zu schaffen werde ich im Folgenden kurz die verschiedenen kontinuierlichen Nierenersatzverfahren und ihre gebräuchlichen Abkürzungen in Anlehnung an (Hörl 2004) in Tab. 5 entschlüsseln:

\begin{tabular}{|c|c|}
\hline \multicolumn{2}{|c|}{ Arteriovenöse Formen } \\
\hline $\begin{array}{c}\text { SCUF } \\
\text { CAVH } \\
\text { CAVHD }\end{array}$ & $\begin{array}{l}\text { Slow continuous ultrafiltration } \\
\text { Kontinuierliche arteriovenöse Hämofiltration } \\
\text { Kontinuierliche atreriovenöse Hämodialyse }\end{array}$ \\
\hline \multicolumn{2}{|c|}{ Venovenöse Formen } \\
\hline $\begin{array}{l}\text { CVVH } \\
\text { CVVHD } \\
\text { CVVHDF }\end{array}$ & $\begin{array}{l}\text { Kontinierliche venovenöse Hämofiltration } \\
\text { Kontinuierliche venovenöse Hämodialyse } \\
\text { Kontinuierliche venovenöse Hämodiafiltration }\end{array}$ \\
\hline \multicolumn{2}{|r|}{ Hybridformen } \\
\hline $\begin{array}{l}\text { SLEDD } \\
\text { EDD }\end{array}$ & $\begin{array}{l}\text { Slow efficient daily dialysis } \\
\text { Extended daily dialysis }\end{array}$ \\
\hline
\end{tabular}

Tab.5 Dialyse und Hämofiltrationsformen

\subsubsection{Nierenersatztherapie beim ANV}

Beim unkomplizierten ANV ist die intermittierende Hämodialysebehandlung die Therapie der Wahl (Hörl 2004). Allerdings bekommen die kontinuierlichen Verfahren einen wichtigen Stellenwert bei komplizierterem, intensivpflichtigem Nierenversagen. Bei hämodynamisch instabilen, hyperkatabolen oder Patienten mit vermehrter Infusions- und Ernährungsbedürftigkeit ist die Indikation zu kontinuierliche Verfahren gegeben. Durch den hier langsamen stattfindenden Volumenshift zwischen den Kompartimenten werden hypovolämischen Situationen abgemildert oder vermieden, was sich in einer höheren Kreislaufstabilität widerspiegelt. Wenn konventionelle Verfahren in diesen Fällen nicht zu Verfügung stehen werden zunehmend Hybridmethoden wie SLEDD und EDD angewandt. Die in dieser Arbeit betrachteten Patienten wurden im Falle der Notwendigkeit einer Nierenersatztherapie alle mit kontinuierlicher Hämofiltration behandelt. Wie oben bereits erwähnt, wird bei der Entwicklung einer progredienten Azotämie ein prophylaktischer Dialysebeginn bereits vor Auftreten von Symptomen befürwortet (Hörl 2004). Die Aussagen 
zur Peritonealdialyse sind in der Literatur teils widersprüchlich. Laut (Harrison et al. 2005) sind Peritonealdialyse und Hämodialyse in der Therapie des ANV gleichwertig und von individuellen Bedürfnissen wie hämodynamischer Instabilität oder großer Operationen mit Eröffnung des Peritoneums abhängig, wohingegen Hörl (2004) die Peritonealdialyse bei Erwachsenen als unbedeutend und nur als wichtige Behandlungsoption bei Kindern einstuft.

\subsubsection{Prognose des ANV}

Die Letalität des ANV beträgt ca. 50\% (Harrison et al. 2005) und hat sich in den letzten drei Jahrzehnten kaum verändert. Allerdings ist dabei zu beachten, dass das ANV als multifaktorielles Geschehen hinsichtlich der Letalität meist durch die verursachende Grunderkrankung und deren Folgeerkrankungen bestimmt ist. Die Mortalität von Patienten mit ANV ist im Vergleich zu einem passenden Kontrollkollektiv bis zu fünffach erhöht (Harrison et al. 2005). Somit ist das ANV mit seinen Nebenwirkungen und Komplikationen ein unabhängiger Risikofaktor für die Mortalität. Die Mortalitätsraten schwanken je nach Ursache des ANV zwischen $15 \%$ bei geburtshilflichen Komplikationen, $30 \%$ bei toxischem ANV und bis zu $60 \%$ bei Traumata und großen Operationen wie Herzoperationen (Harrison et al. 2005). Faktoren, die eine schlechte Prognose andeuten, sind eine Oligurie von unter 400 $\mathrm{ml}$ pro Tag schon bei Diagnosestellung und ein Anstieg des Serumkreatinins über $3 \mathrm{mg} / \mathrm{dl}$. Alte und multimorbide Patienten haben eine erhöhte Mortalität. Glücklicherweise haben die meisten ein ANV überlebenden Patienten nachher eine ausreichende Nierenfunktion um ein normales Leben zu führen, obwohl ca. 30\% ein Residuum, wie eine subklinische Beeinträchtigung oder eine Narbe aufweisen (Harrison et al. 2005). Ungefähr 5\% benötigen allerdings eine langfristige Nierenersatztherapie oder Nierentransplantation, da sie sich nicht von dem ANV erholen. 


\subsection{Stand der Forschung zum ANV nach kardiochirurgischen Eingriffen}

Das ANV ist eine der schwerwiegensden Komplikationen in der offenen Herzchirurgie. Obwohl sich einige perioperative Bedingungen wie die Modernisierung anästhesiologischer Verfahren, neue Dialysetechnologien und biokompatible Dialysemembranen verbessert haben, ist die Mortalität des ANV nach Herzoperationen seit einigen Jahrzehnten weitgehend konstant geblieben (Landoni et al. 2006). Ein möglicher Grund dafür könnte sein, dass zunehmend ältere und durch breitgefächerte Grunderkrankungen belastete Patienten der Herzchirurgie zugeführt werden. Des Weiteren ist das Verständnis der Pathogenese und der damit verbundenen Präventions- und Behandlungsmöglichkeiten des ANV noch unzureichend.

Die Inzidenz des ANV nach Herzchirurgie wird in der Literatur mit 0,7-31\% angegeben (Thakar et al. 2003b). Chertow et al. (1989) definieren beispielsweise das ANV als einen Anstieg des Serumkreatinins von 50\% über den Ausgangswert. Unter Zugrundelegen dieser Definition beträgt die Inzidenz des ANV bei Allgemeinpatienten 5\% im Vergleich zu Herzchirurgischen Patienten mit 31\%. Die Bedeutung des ANV begründet sich vor Allem in den hohen Mortalitätsraten. Nach herzchirurgischen Operationen zeigt sich ein Mortalitätsrisiko von bis zu 90\% (Landoni et al. 2006). Andere Autoren beschreiben eine Mortalität, die 50-60\% übersteigt (Garwood et al. 2004). Das ANV ist hierbei unabhängig, auch nach Korrektur von Komorbidität und postoperativen Komplikationen, mit einer erhöhten Mortalität nach Eingriffen am Herzen mit Herz-Lungen-Maschine (HLM) assoziiert. Trotz dieses unabhängigen Zusammenhangs scheint die die insgesamt hohe Mortalität des ANV durch die Tatsache bedingt zu sein, für weitere schwere Organdysfunktionen zu prädestinieren und es ist davon auszugehen, dass als Grund für die hohe Mortalität nicht das ANV isoliert, sondern das mit ihm verbundene multifaktorielle Geschehen und seine Komplikationen verantwortlich sind (Garwood et al. 2004). Die Forschung bezüglich des ANV im Rahmen von Herzoperationen beschäftigt zum Einen mit der Detektion von risikobehafteten Patienten, mit dem Ziel frühzeitig kompensatorisch-therapeutische Maßnahmen ergreifen zu können. Des Weiteren spielen das Verständnis der komplexen Pathopysiologie des ANV und der Einflüsse der Variablen, die mit der Herz-LungenMaschine assoziiert sind, eine zentrale Rolle. Die Erforschung und Erprobung nephroprotektiver Medikamente, die das Auftreten eines ANV verhindern oder abschwächen könnten, stellen einen weiteren Ansatzpunkt dar. 
Die Mechanismen, die zur Entstehung eines prärenalen Nierenversagens, das im Zuge einer prolongierten Ischämie zu einer akuten Tubulusnekrose führen kann, beitragen, spielen auch für die Pathophysiologie des ANV nach herzchirurgischen Operationen eine maßgebliche Rolle. Allerdings sind die Einflussfaktoren im Vergleich zu einem prärenalen ANV bei Exsikkose oder starkem Blutverlust mit einer hämodynamischen Erklärung der Kompromittierung der Nierendurchblutung- und Funktion wesentlich breitgefächerter. So ist bei der durch den Einsatz der HLM verursachten Veränderung der Hämodynamik nicht nur ein Volumenverlust und eine Veränderung der Hämodynamik, sondern weitere Besonderheiten bedeutsam für die Entstehung des ANV. Allgemein geht man davon aus, dass die HLM zu einer Minderperfusion der Niere führt. Neben dem zusätzlich veränderten nicht physiologisch-pulsatilen Blutfluss, welcher ebenfalls in einer unzureichenden Nierenperfusion resultieren kann, den regionalen Veränderungen bei Perfusion mit HLM und den Auswirkungen der Hypothermie, spielen aber noch weitere Mechanismen eine Rolle. Man vermutet, dass embolische Phänomene einen weiteren Aspekt der Genese des ANV darstellen könnten (Garwood el al. 2004). Luft, Thrombozyten oder atheromatöse Plaque aus der Aorta könnten durch die Manipulationen im Rahmen der Operation in die Blutbahn und konsekutive in die Nierengefäße gelangen und aufgrund der Ischämie schon initiierte Prozesse noch weiter aggravieren. Ein weiterer wichtiger Mechanismus ist die Betrachtung der HLM als Fremdkörper mit Oberflächeneigenschaften, die im Blut verschiedenste Kaskadensysteme wie das Gerinnungssystem, das Komplementsystem, das Kallikrein-Kinin-System und die Entstehung von freien Sauerstoffradikalen (Garwood et al. 2004) auslösen. Durch inflammatorische Zytokine wird eine systemische inflammatorische Reaktion des Körpers getriggert (SIRS = systemic inflammtory response syndrome), welche für die Niere eine Gefährdung darstellen und eine wesentliche Rolle bei der Entstehung des ANV spielen könnten .

Inwieweit die HLM und deren Management wirklich Einfluss auf das postoperative Entstehen eines ANV haben, wird in der Literatur kontrovers diskutiert und stellt den Schwerpunkt dieser Arbeit dar. Zwar kommen Lema et al. (1995) zu dem Schluss, dass die Nierenfunktion nicht nachteilig durch die HLM beeinflusst ist, jedoch belegen viele Studien eindeutige Zusammenhänge zwischen HLM-Parametern und dem Auftreten des postoperativen ANV. Die Dauer der HLM beispielsweise wird von vielen Studien als unabhängiger Faktor für das ANV bewertet (Conlon et al. (1999), Fischer et al. (2003), Heikkinen et al. (1985), Mangos et al. (1995), Thakar et al. (2005)). Boldt et al. (2003) beschreiben den signifikanten Unterschied der tubulären Schädigung zwischen HLM-Zeiten von über 90 Minuten im Vergleich zu HLM- 
Zeiten unter 70 Minuten, welche mit deutlich niedrigeren Markern für eine tubuläre Schädigung verbunden sind. Als Marker für die Schädigung wurden N-acetyl-beta-Dglucosaminidase, alpha 1-mikroglobulin, glutathion-transferase-pi und glutathion-transferasealpha nach der Einleitung der Anästhesie, am Ende der Operation und am ersten und zweiten postoperativen Tag auf der Intensivstation gemessen. Bei Patienten mit einem dialysepflichtigen ANV wurden von Fischer et al. (2002) im Vergleich zu Patienten mit erhöhtem Serumkreatinin ohne Dialysebedarf und Patienten ohne Serumkreatininanstieg neben einer längeren HLM-Dauer auch eine niedrigere Flussrate der extrakorporalen Zirkulation und längere Perioden mit HLM-Perfusionsdrücken unter $60 \mathrm{~mm} / \mathrm{Hg}$ festgestellt. Garwood et al. (2004) haben als intraoperative Risikofaktoren die Kombination einer Revaskularisierungsoperation mit einer weiteren intrakardialen Prozedur wie zum Beispiel ein Klappenersatz, eine HLM-Zeit von mehr als $3 \mathrm{~h}$ und verschiedene Indikatoren für ein vermindertes Herzzeitvolumen (HZV) wie den Bedarf an inotropen Medikamenten oder IABP (intra-aortale Ballonpumpe) herausgefunden. Andere Studien erhärten die Überlegenheit einer am schlagenden Herzen durchgeführten Revaskularisierungsoperation im Vergleich zu konventioneller Revaskularisierung mit HLM und kardioplegischem Arrest (Ascione et al.(1999), Rastan et al. (2006)). Auch Beauford et al. (2004) beschreibt durch das Vermeiden der HLM eine niedrigere Inzidenz des ANV und ein verbessertes Krankenhausüberleben. Dem gegenüber beschreiben Chukwuemeka et al. (2005) in einer Studie mit 2869 Patienten, dass die Durchführung von Operationen am schlagenden Herzen nicht mit einem erniedrigten Risiko für ein Nierendysfunktion assoziiert ist, und die Nierenfunktion kein Entscheidungskriterium für die Entscheidung zwischen konventioneller und Operation am schlagenden Herzen sein sollte. Im Tierversuch konnte der Vorteil einer biologisch-variablen Perfusion mit Schlag zu Schlag Variabilität nachgewiesen werden, indem im Vergleich zu einer Kontrollgruppe mit nichtpulsatiler Perfusion diverse Marker für eine tubuläre Schädigung erniedrigt, die Urinausscheidung erhöht und die Hypothermiedauer verkürzt waren. Im Tierversuch ist auch die Temperatur für den kortikalen Blutfluss von Bedeutung. Allerdings sind diese Erkenntnisse noch nicht am Menschen nachvollzogen worden. Eine regionale myokardiale Azidose während der Abklemmzeit der Aorta allerdings konnte von Kumbhani et al. (2005) als unabhängiger Faktor für das 30-Tage-Überleben nach Herzoperationen definiert werden. Auch die Art der Operation scheint eine Rolle zu spielen. So beschreibt Heikkinen et al. (1985) das höhere Risiko von Klappenoperationen im Vergleich zu reinen Revaskularisierungsoperationen. Da das Management der HLM immer noch nicht evidenzbasiert, die Studienlage teils widersprüchlich ist, die neuen 
pathophysiologischen Erkenntnisse aber auf eine wichtige Stellung der HLM-Parameter in der Entstehung des ANV hinweisen, lässt sich zusammenfassend sagen, dass weitere Studien notwendig sind um die Zusammenhänge besser zu verstehen und Verbesserungen umsetzen zu können. Neben den HLM-assoziierten Faktoren wie Perfusionszeit, Ischämiezeit, nichtpulsatilem Fluss, Hypoperfusion, Hypothermie, Klappen oder Revaskularisierungsoperation, myokardialer Azidose und SIRS wurden in zahlreichen Studien andere Faktoren identifiziert, die in signifikantem Zusammenhang mit dem postoperativen Auftreten des ANV stehen. Conlon et al. (1999) unterscheidet bei der Einteilung von Risikofaktoren zwischen akutem Nierenversagen mit Dialyseindikation (ANV-D) und ANV ohne Dialyse aber Serumkreatininanstieg von $1 \mathrm{mg} / \mathrm{dl}$ über der Ausgangslinie (ANV). Für das ANV-D sind hohes Alter, erhöhtes Serumkreatinin, die HLM-Dauer, Diabetes, reduzierte Ejektionsfrakton des Herzens und erhöhtes Körpergewicht Risikofaktoren. Unter Ihnen sind erhöhtes Serumkreatinin, HLM-Dauer, und Diabetes unabhängige Risikofaktoren für das ANV-D. Ein klinischer Risikoscore, der beide Geschlechter und Hochrisikopatienten einbezieht und außerdem eine Abstufung der Risikofaktoren anbietet ist die die Studie von Thakar et al. (2005). Sie nennen folgende Risikofaktoren: Alter, weibliches Geschlecht, Herzinsuffizienz, präoperativer Einsatz von IABP, Notoperation, Zustand nach Herzoperation, COPD, Diabetes mellitus, präoperatives Serumkreatinin, HLM-Zeit und Art der Operation. Im Jahr 2006 erweitert bzw. bestätigen Landoni et al. (2006) folgende Parameter, welche in signifikantem Zusammenhang mit dem Auftreten des ANV stehen: Notfall-OP, präoperative Niereninsuffizienz, Einsatz von IABP, Zustand nach Herzoperation, weibliches Geschlecht, geringe Ejektionsfraktion des Herzens, Blutung über 1000ml, COPD und das Alter. Wijeysundera et al. (2006) bestätigen den Zusammenhang von präoperativer Nierenfunktionseinschränkung und postoperativ benötigter Nierenersatztherapie auch bei latenter Nierenfunktionsstörung mit noch normalem Serumkreatinin mit einer Kreatininclearance unter $60 \mathrm{ml} / \mathrm{min}$. Daher schlägt er wie auch Noyez et al.(2006) zur besseren Evaluation der Nierenfunktion die Formel nach Cockroft-Gault zu Errechnung der Kreatininclearance und damit Abschätzung der GFR vor. Der Einsatz von Medikamenten die den renalen Blutfluss erhöhen oder sich in anderer Weise positiv auf die Nierenfunktion auswirken wird in der Literatur ebenfalls kontrovers diskutiert und hat einige Veränderungen erfahren. Die in Tierversuchen so vielversprechende Verabreichung von niedrig dosiertem Dopamin hatte viele Hoffnungen geweckt, konnte aber in seiner Wirkung bei Menschen nicht bestätigt werden. Viele Studien belegen mittlerweile, dass Dopamin ein ANV nicht verhindern kann (Bellomo et al. 1994, Garwood et al. 2004). Furosemid hat bei kurzlebigen 
oligurischen Nierenfunktionseinschränkungen ohne Indikation zur Dialyse zur Aufrechterhaltung der Diurese noch seine Berechtigung. Neben der Tatsache, dass die Urinausscheidung den postoperativen Zustand der Niere nach Herzoperation nicht maßgeblich bestimmt, (Garwood et al. 2004) sind einige schädigende Wirkungen von Furosemid in Bezug auf die Nierenfunktion deutlich geworden und es sollte daher zurückhaltend eingesetzt werden. Neuere Substanzen wie BNP (Brain natriuretic peptide) und Vasopeptidase Inhibitoren werden zur Zeit erprobt und gelten als vielversprechende Alternativen für die Zukunft. 


\section{Methodik}

\subsection{Patientengruppe}

In diese Studie gingen die Daten von 3574 Patienten ein, die sich in der Klinik für Thorax-, Herz- und Gefäßchirurgie der Universitätsklinik Göttingen einer Operation am offenen Herzen unter Einsatz einer extrakorporalen Zirkulation unterziehen mussten. Der Zeitraum der Erhebung der Patientendaten erstreckt sich vom 03.01.2000 bis zum 30.12.2005. Es wurden nur erwachsene Patienten ab dem 18. Lebensjahr einbezogen, welche fast alle aus dem Landkreis Göttingen und den an Göttingen angrenzenden Landkreisen stammten. Einen ersten Überblick über die Patientengruppe hinsichtlich der Geschlechterverteilung, Art der Operation und Notwendigkeit eines Nierenersatzverfahrens bzw. Auftreten eines akuten Nierenversagens stellt folgende Graphik dar.

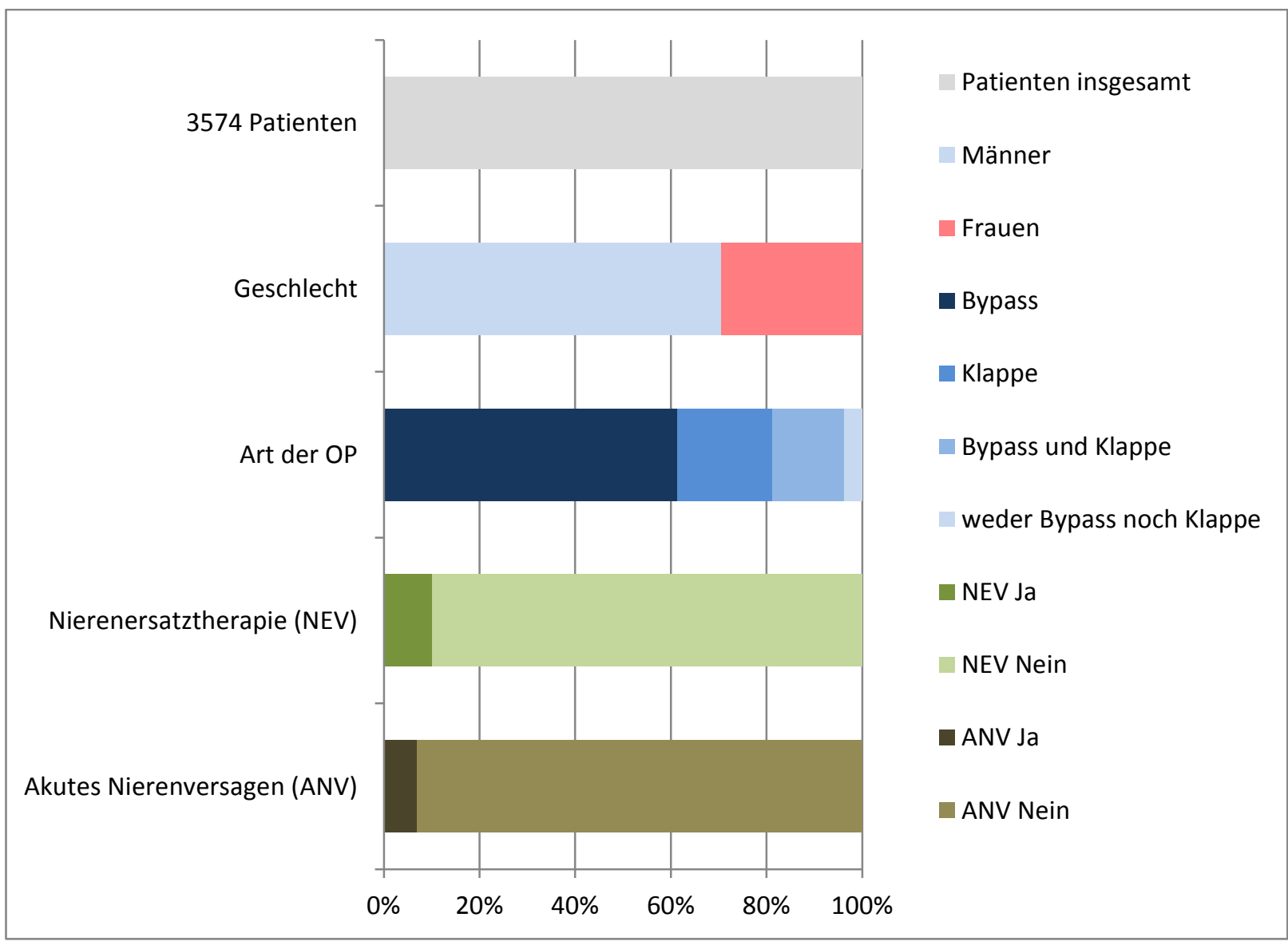

Abb. 3 Übersicht über die Patientengruppe

Von den 3574 operierten Patienten waren 2522 Männer und 1052 Frauen. Die Operationen selbst gliederten sich folgendermaßen in Subgruppen auf: 2190 Patienten unterzogen sich einer reinen Revaskularisierungsoperation ohne Manipulation an Herzklappen oder anderen 
intrakardialen Prozeduren. 714 Patienten benötigten ausschließlich eine Operation an einer Herzklappe ohne Revaskularisierung der Koronargefäße. 537 Patienten wurden in einer Operationssitzung kombiniert an einer oder mehreren Herzklappen und den Koronargefäßen im Sinne einer Revaskularisierung operiert. Eine kleinere Anzahl von 133 Patienten wurde weder an den Herzklappen noch an den Koronargefäßen operiert und betrifft Operationen wie den Verschluss von persistierendem Foramen ovale oder eines Atriumseptumdefektes und Operationen zum Ersatz von aszendierender oder deszendierender Aorta oder der Resektion von Aneurysmata im Herzen oder der Aorta. In der vorliegenden retrospektiven Studie befinden sich 365 Patienten, bei denen aufgrund eines akuten Nierenversagens eine Nierenersatztherapie in Form von Hämofiltration eingeleitet werden musste, was als manifestes ANV gewertet wird. Dieses Kollektiv wird in dieser Studie als Gruppe I (ANV-H) bezeichnet und den nicht hämofiltrationspflichtigen Patienten im Hinblick auf die zu untersuchenden Parameter gegenüberstellt. Die Indikation zur Nierenersatztherapie wird auf den Intensivstationen im Universitätsklinikum Göttingen durch eine Standardarbeitsanweisung SOP (=Standard Operating Procedure) geregelt, welche vom Zentrum Anaesthesiologie, Rettungs- und Intensivmedizin im Namen von Prof. Dr. M. Quintel herausgegeben wird und für die dieser Studie zugrunde liegenden Daten zum letzten Mal am 01.06.2007 aktualisiert wurde. Laut dieser Anweisung soll eine Nierenersatztherapie beginnen, bevor die folgenden Komplikationen auftreten:

- Lungenödem oder drohende rechtsventrikuläre Dekompensation durch Überwässerung, welche nicht durch Diuretika behandelt werden kann

- Urämische Komplikationen (Enzephalopathie, Neuropathie, Perikarditis, Azidose mit $\mathrm{pH}<7,0$

- Hyperkaliämie ( $>6,5 \mathrm{mmol} / \mathrm{l})$

Es gilt zu beachten, dass wie im Punkt 1.3.7 beschrieben, der Einsatz von Nierenersatzverfahren nicht einheitlich geregelt ist, sondern ein multifaktorielles Konzept darstellt, welches auf eine individuelle klinische Bedarfssituation abgestimmt wird. Wie aus obigen Ausführungen hervorgeht, wird der Serumkreatininwert als Marker für die Ausscheidungsfunktion der Niere als direkter Entscheidungsfaktor für die Indikationen zur Nierenersatztherapie nicht herangezogen. Für das von uns als Gruppe II (ANV) bezeichnete Kollektiv stellt er aber ein sinnvolles Kriterium zur Beurteilung der Nierenfunktion dar. Um den Kreatininwert noch aussagekräftiger zu machen und das Nierenversagen im Sinne der RIFLE-Kriterien definieren zu können, haben wir die Serumkreatininwerte mit Hilfe der 
Formel von Cockcroft und Goult $\left(\mathrm{Cl}_{\mathrm{Krea}}=[(150-\mathrm{LA}) \cdot \mathrm{KG} /\right.$ Serumkreatinin $]-\mathrm{a}$; siehe Punkt 1.3.5) umgerechnet. Dies liefert eine annähernde Einschätzung der tatsächlichen glomerulären Filtrationsrate, welche Lebensalter, Gewicht und Geschlecht des Patienten einbezieht (Wijeysundera et al. 2006). Eine noch genauere Beurteilung der GFR mithilfe der Berechnung der Kreatininclearance war mit unseren Daten nicht durchführbar, da in der Routine kein Urin über 24h gesammelt wurde. 251 Patienten wiesen einen Anstieg des Serumkreatinins bzw. einen Abfall der GFR im Sinne der RIFLE-Kriterien auf, was für das Kriterium F = Failure (Versagen) als ANV zu werten ist. Diese Patienten, welche wir der Gruppe II zuordneten, haben in dieser Studie also definitionsgemäß ein ANV.

Der Einsatz von Nierenersatzverfahren in der Gruppe I ist ein relativ sicheres Zeichen für ein ANV, weil neben Laborwerten auch klinische Variablen wie die Retention von Flüssigkeit, Oligurie und Symptome einer Azotämie bzw. Urämie einbezogen werden. Die Einteilung des ANV der Gruppe II wird über ein um den Faktor 3 erhöhtes Serumkreatinin oder einen Abfall der GFR um 75\% oder einen Serumkreatininspitzenwert von $4 \mathrm{mg} / \mathrm{dl}$ im Sinne der RIFLE Kriterien definiert. (Siehe Tab.6)

\begin{tabular}{|l|ll||}
\hline Gruppe I $(A N V-H)$ & & \\
& & Einsatz von Nierenersatzverfahren \\
\hline & & \\
\hline Gruppe II $(A N V)$ & gemäß RIFLE-Kriterien (Siehe Tab.1) \\
& erhöhtes Serumkreatinin um Faktor 3 oder \\
& - Abfall der GFR um 75\% oder \\
& - Serumkreatininwert von $4 \mathrm{mg} / \mathrm{dl}$ \\
\hline
\end{tabular}

Tab.6 Gruppeneinteilung des ANV

\subsection{Datensatz}

Zur Erstellung des Datensatzes wurden Patientendaten aus zwei Datenbanken des Universitätsklinikums Göttingen extrahiert. Das Ausgabeformat war in allen Fällen comma separated value (CSV). Die erste Datenbank, GISI (Göttinger Informationssystem für Intensivmedizin), wird auf der Intensivstation eingesetzt, um Daten von Patienten zu erfassen, die dort behandelt wurden. In GISI wurde als Merkmal „Art der Operation“ für die Patienten nach Herzoperation das Merkmal „Herz-Operation“ eingegeben. Da alle Patienten nach einer offenen Herzoperation auf die Intensivstation verlegt werden, sind mit Sicherheit alle operierten Patienten auch tatsächlich in der Intensivdatenbank eingetragen, von Eingabefehlern- und Ausfällen abgesehen. Aufgrund der Tatsache, dass unsere Abfrage sehr viele Parameter umfasste, gepaart mit der beschränkten Rechenkapazität der Computer 
bezüglich dieser enormen Datenmenge, war es nötig die Abfrage pro Intensivstation pro Jahr in drei Teilschritten erfolgen zu lassen. Im Universitätsklinikum Göttingen gibt es zwei Intensivstationen, auf die am Herzen operierte Patienten verlegt werden können, nämlich die Station 0117 und 0118. Vor November 2003 allerdings wurde die Station 0117 in zwei Abschnitte eingeteilt, nämlich 0111 und 0112 ebenso wie die Station 0118, welche sich aus der Station 0113 und 0115 zusammensetzte. Für die Stationen 0117 und 0118 wurden also jeweils drei Datensatzabfragen gemacht, um die unten in der Tabelle aufgeführten Parameter zu jedem Patienten zu erhalten. Um Informationen über Parameter der extrakorporalen Zirkulation mit der Herz-Lungen-Maschine (HLM) zu erhalten, wurde eine weitere Datenbank als Quelle genutzt, welche GISI-OP heißt und im Operationssaal eingesetzt wird, um technische Daten der HLM während der Operation aufzuzeichnen. Das Ausgabeformat war hier ebenfalls comma separated value (CSV). Die Parameter aus diesen Abfragen sind ebenfalls in untenstehender Tabelle zu sehen. Auch der Name des Patienten musste zur Verknüpfung der verschiedenen Datensätze eines Patienten herangezogen werden, da das Geburtsdatum in Anbetracht der Datenmenge keine sicheres Kriterium darstellte und patientenbezogenen Marker, wie Pat.-Nr., Fall-Nr oder OP-Nr. nicht durchgehend in allen Datenbanken erfasst wurden. Anschließend wurde der Datensatz aus Gründen des Datenschutzes anonymisiert. Einige Parameter wurden aus dem Datensatz entfernt, weil sie nur dazu dienten bestimmte Abfragen zu identifizieren oder weil sie in Bezug auf die Fragestellung in unserer Studie unbrauchbar wurden. Die in unten stehender Tabelle (Tab.7) aufgeführten Parameter stellen also die Ausgangssituation der Datenabfrage vor Erstellung des endgültigen Datensatzes dar.

\begin{tabular}{|c|c|c|}
\hline Name der Datenbank & $\mathrm{Nr}$ & Parameter \\
\hline \multirow{3}{*}{$\begin{array}{c}\text { GISI } \\
\text { (Station 0118) }\end{array}$} & 1. & $\begin{array}{l}\text { Name;Vorname;Geschlecht;Alter;PNR;Größe;Gewicht; } \\
\text { Operation;Diagnose;Risikofaktoren;Neben- } \\
\text { Diagnosen;Vorerkrankungen;Art d. Operation }\end{array}$ \\
\hline & 2. & $\begin{array}{l}\text { Name;Vorname;PNR;IABP;KreatininA;KreatininB; } \\
\text { Urinausscheidung;Gesamtbilanz;Hämofiltration;Filtrations } \\
\text {-dauer;PerfusorenA;PerfusorenB }\end{array}$ \\
\hline & 3. & $\begin{array}{l}\text { Name;Vorname;PNR;FNR;Art d. } \\
\text { Operation;Aufnahme;Verlegung; } \\
\text { OP-Datum;Exitus;Suprarenin max;Arterenol max; } \\
\text { Perfan max }\end{array}$ \\
\hline $\begin{array}{c}\text { GISI } \\
\text { (Station 0117) }\end{array}$ & 4. & $\begin{array}{l}\text { Name;Vorname;Geschlecht;Alter;PNR;Größe;Gewicht; } \\
\text { Operation;Diagnose;Risikofaktoren;Neben- } \\
\text { Diagnosen;Vorerkrankungen;Art d. Operation }\end{array}$ \\
\hline
\end{tabular}




\begin{tabular}{|c|c|c|}
\hline & 5. & $\begin{array}{l}\text { Name;Vorname;PNR;IABP;KreatininA;KreatininB; } \\
\text { Urinausscheidung;Gesamtbilanz;Hämofiltration;Filtrations } \\
\text {-dauer;PerfusorenA;PerfusorenB }\end{array}$ \\
\hline & 6. & $\begin{array}{l}\text { Name;Vorname;PNR;FNR;Art } \\
\text { d.Operation;Aufnahme;Verlegung; } \\
\text { OP-Datum;Exitus;Suprarenin max;Arterenol max; } \\
\text { Perfan max }\end{array}$ \\
\hline $\begin{array}{c}\text { GISI } \\
\text { Blutverluste } \\
\text { (Station } 0117 \text { und 0118) } \\
\end{array}$ & 7. & $\begin{array}{l}\text { Name;Vorname;PNR;FNR;Aufnahme;OP-Tag;OP-Tag+1; } \\
\text { OP-Tag+2;OP-Tag+3;OP-Tag+4;OP-Tag+5;OP-Tag+6 }\end{array}$ \\
\hline \multirow[t]{2}{*}{ GISI-OP } & 8. & $\begin{array}{l}\text { Name;Vorname;OP- } \\
\text { Tag;PNR;Größe;Gewicht;Geschlecht;OP- } \\
\text { Dauer;Ischämiezeit;Perfusionszeit;Reperfusionszeit; } \\
\text { Temperatur;Art d.Pumpe;Kardioplegie;Alter }\end{array}$ \\
\hline & 9. & $\begin{array}{l}\text { Name;Vorname;Geburtsdatum;OP-Datum;OP- } \\
\text { Team;Diagnose;Operation }\end{array}$ \\
\hline
\end{tabular}

Tab.7 Datensatzentstehung

(PNR=Patientennummer; FNR=Fallnummer; IABP=intraaortale Ballonpumpe)

Um einen einzigen gemeinsamen Datensatz zu erstellen, der für jeden einzelnen Patienten alle erwünschten Werte aus beiden Datenquellen vereint, wurden Microsoft Excel, Microsoft Access inklusive „Makros“ ( = programmierbare Funktionen innerhalb der genannten Programme) und einige mittels der Programmiersprache (PHP) selbst programmierte Systeme verwandt. Im Zuge dessen wurde von der in der Tabelle dargestellten Datenlage schrittweise Datensätze zusammengeführt bis letztendlich ein einziger Datensatz erstellt war, der alle 5 Jahre und alle Parameter umfasste und anschließend statistisch ausgewertet wurde. Die Datensätze 1-9 wurden nacheinander eingelesen und anhand von Parametern verglichen. Ausgehend vom CSV- Format wurden die Daten durch eine in PHP programmierten Befehl zeilenweise in Arrays umgewandelt und so in einer bestimmten Weise dargestellt und mit den anderen Datensätzen vergleichbar gemacht. PHP bezeichnet ein rekursives Akronym für „Hypertext Preprocessor“ und ist eine Skriptsprache mit einer an „C“ bzw. „C++“ angelehnten Syntax, die sich für die Programmierung in Datenbanken gut eignet. Einen Array kann man am besten als Beschreibung einer Datenstruktur, welche die Anordnung von Einträgen definiert, bezeichnen. Das Format der Eintragungen wurde also durch diese Arrays festgelegt und die Daten dann zeilenweise ausgelesen und miteinander verglichen. Die Patientennummer, Fallnummer, der Name und Nachname und das Datum der Aufnahme und Operation dienten dabei teils individuell, teils in Kombination miteinander als Vergleichskriterium. Dieses Vorgehen wurde mit dem Institut für medizinische Statistik in 
Göttingen diskutiert und als korrekt bewertet. Auf diese Art und Weise wurden zunächst die Datensätze 1, 2 und 3 sowie 4, 5 und 6 zusammengefasst. Nachdem die Informationen aus den Datensätzen 8 und 9 ebenfalls zu den entsprechenden Patienten zusammengeführt wurden, konnten die dabei entstehenden Datensätze jeweils miteinander verknüpft werden, so dass nur noch 1 Datensatz und die Abfrage der Blutverluste übrig blieben. Diese beiden Datensätze enthielten beide dieselbe Fallnummer und waren deshalb leicht einander zuzuordnen. Bei allen Arbeitsschritten wurden nicht eindeutige und aufgrund von Eingabefehlern falsche, unsinnige oder fehlende Einträge in ihrer Gesamtheit, d.h. mit allen diesen Patienten betreffenden Zusatzinformationen aus dem Datensatz entfernt. Ein anderes Vorgehen war aufgrund der großen Datenmenge nicht möglich. Es liegt dem Verlust von Datenmaterial aber kein systematischer Fehler zugrunde, sondern ein gleichmäßig verteiltes Muster von menschlichen Eingabefehlern über die Jahre hinweg. Im Anschluß an diese Korrekturen wurden alle Patienten unter 18 Jahre gelöscht, da sich unsere Studie nur auf Erwachsene ab dem 18. Lebensjahr bezieht, und ein finaler Datensatz erzeugt. Der von uns statistisch ausgewertete endgültige Datensatz umfasst somit insgesamt 3574 Patienten.

\subsection{Statistik}

In der statistischen Auswertung wurden folgende Parameter auf einen Zusammenhang mit dem Auftreten eines postoperativen ANV untersucht:

\section{Kategorielle Parameter:}

- Geschlecht (W versus M)

- Bypass-Operation (Ja versus Nein)

- Klappen-Operation (Ja versus Nein)

- Re-Operation (Ja versus Nein)

- Einsatz von IABP (Ja versus Nein)

- Art der Pumpe (Zentrifugal versus Rollerpumpe)

- Art der Kardioplegie (Bretschneider versus Blutkardioplegie)

- Temperatur ( Normothermie versus milde bzw. Tiefe Hypothermie)

- Art der Operation ( Bypass versus Klappe versus Kombination versus Andere)

2. Metrische Parameter

- Alter (Jahre)

- Kreatinin A bzw. B (mg/dl)

- Operationsdauer (min) 
- Ischämiezeit (min)

- Perfusionszeit (min)

- Minimale Temperatur $\left({ }^{\circ}\right.$ Celsius $)$

- Blutverlust (ml) auch im Hinblick auf zeitlichen Verlauf und Wechselwirkung

Jeder dieser Parameter wurde für die Gruppe I (Einsatz von Nierenersatzverfahren) und Gruppe II (ANV gemäß RIFLE) auf Signifikanz untersucht. Um auch latentere Zusammenhänge zwischen diesen Parametern und der Beeinflussung der Nierenfunktion zu erfassen, wurde zusätzlich eine Untersuchung der Parameter im Hinblick auf den absoluten Kreatininanstieg durchgeführt.

Im ersten Teil wurden die Einflussgrößen auf die Notwendigkeit eines Nierenersatzverfahrens (NEV) (Gruppe 1) untersucht. Für die kategoriellen Parameter wurde dabei der $\mathrm{X}^{2}$-Test (ChiQuadrat-Test) verwendet. Für die metrischen Parameter wurde der Wilcoxon-Mann-WhitneyTest angewandt. Speziell die Entwicklung der Blutverluste über die ersten 6 postoperativen Tage der Patienten mit bzw. ohne NEV wurden mit einer nichtparametrischen Varianzanalyse für longitudinale Daten untersucht (Brunner et al. 2002). Hierbei wurde getestet, ob entweder die Zeit oder die definierte Gruppe einen signifikanten Einfluss auf den Blutverlust hat und ob Zeit und Gruppe in einer Wechselwirkung zueinander stehen, d.h. ob der zeitliche Verlauf in den beiden Gruppen verschieden ist.

Im zweiten Teil wurden die Einflussgrößen auf die Definition des ANV nach den RIFLEKriterien in Abgrenzung derer, die laut dieser Definition kein ANV haben, untersucht (Gruppe 2). Für die kategoriellen Parameter wurde dabei der $\mathrm{X}^{2}$-Test verwendet und für die metrischen Daten der Wilcoxon-Mann-Whitney-Test.

Im dritten Teil wurden die Parameter auf einen Zusammenhang mit dem absoluten Anstieg des Serumkreatininwertes untersucht. Hierzu wurde der Kreatininanstieg bei den einzelnen Stufen der kategoriellen Parameter mit dem Wilcoxon-Mann-Whitney-Test im Zweigruppenfall und mit dem Kruskal-Wallis-Test im Mehrgruppenfall verglichen. Der Zusammenhang mit den metrischen Parametern wurde durch den Pearson'schen Korrelationskoeffizienten $\rho$ ermittelt. 
Für alle Tests wurde als Signifikanzniveau $\alpha=5 \%$ festgelegt. Die Analysen der metrischen und kategoriellen Parameter wurden mit der Software R 2.6 (www.r-project.org)

durchgeführt. Für die longitudinale Analyse wurde das Macro „F1_LD_F1“ (www.ams.med.uni-goettingen.de/de/sof/ld/F1_LD_F1.SAS ) für die Software SAS 9.1 (SAS Institute) verwendet. Für die Gruppeneinteilungen und Auszählungen wurde das StatistikProgramm Statistika ${ }^{\circledR}$ benutzt. 


\section{Ergebnisse}

\subsection{Demographische Daten}

In diese Studie gingen die Daten von 3574 Patienten ein, die sich in der Klinik für Thorax-, Herz- und Gefäßchirurgie der Universitätsklinik Göttingen einer Operation am offenen Herzen unter Einsatz einer extrakorporalen Zirkulation unterziehen mussten. Der Zeitraum der Erhebung der Patientendaten erstreckt sich vom 03.01.2000 bis zum 30.12.2005. Es wurden nur erwachsene Patienten ab dem 18. Lebensjahr einbezogen, welche fast alle aus dem Landkreis Göttingen und den an Göttingen angrenzenden Landkreisen stammen.

\subsubsection{Geschlecht der Patienten}

Die Verteilung der Geschlechter in dem Patientenkollektiv zeigt, dass mit $71 \%$ deutlich mehr Männer im Vergleich zu Frauen mit 29\% operiert wurden. Abb. 4 veranschaulicht das prozentuale Verhältnis der Geschlechter.

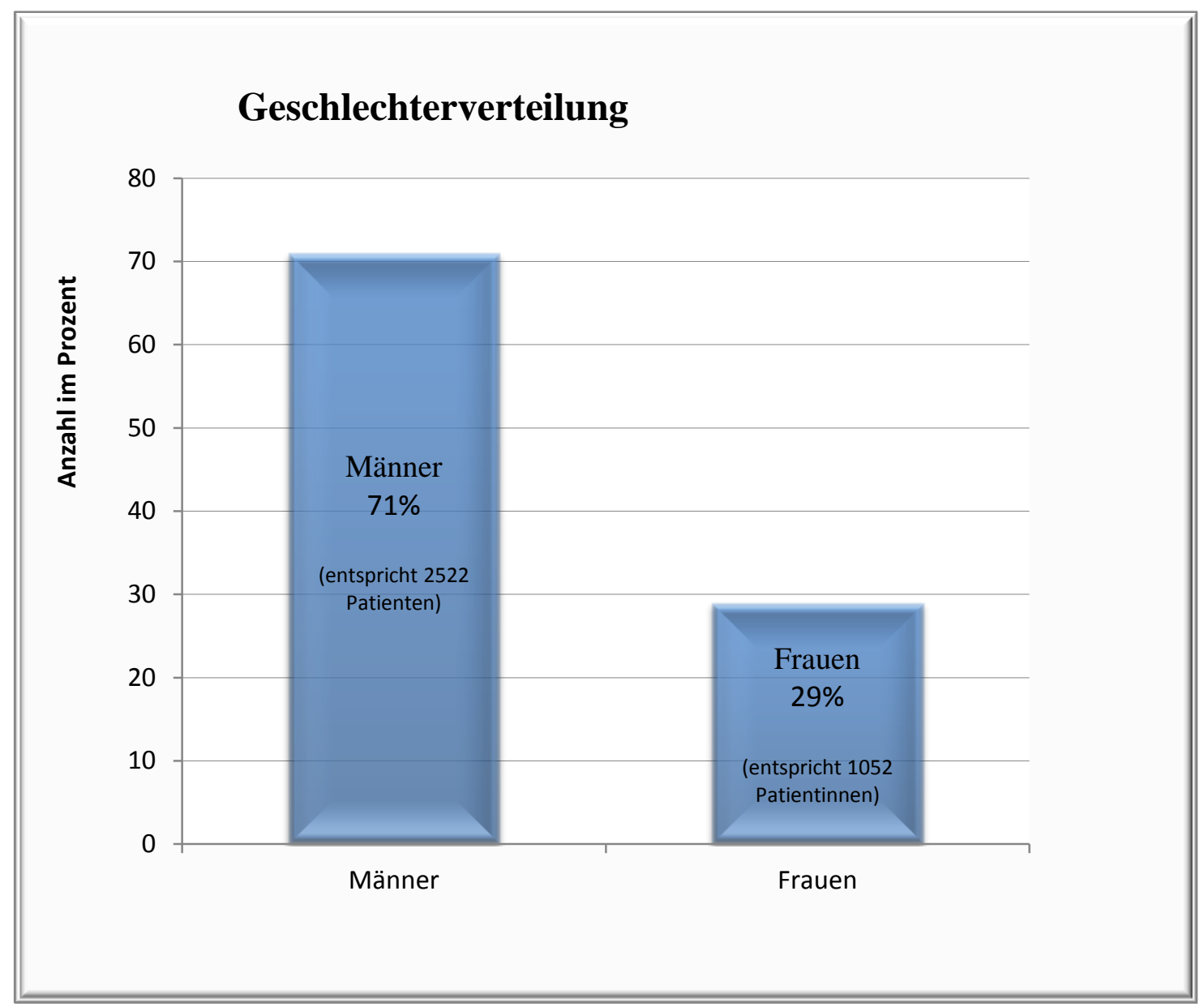

Abb. 4 Geschlechterverteilung 


\subsubsection{Alter der Patienten}

Da unsere Studie nur Erwachsene ab 18 Jahren einbezieht, sind dementsprechend die jüngsten Patienten 18 Jahre alt. Der älteste in unserem Kollektiv operierte Patient war 92 Jahre alt. Abb. 5 zeigt ein Histogramm bezogen auf das Alter der Patienten, welches die absolute Anzahl der Patienten in dem jeweiligen Altersabschnitt zeigt sowie seinen prozentualen Anteil. Aus ihm geht hervor, dass die Patienten zwischen 30 und 40 Jahren nur einen Anteil von $2 \%$ und Patienten zwischen 40 und 50 Jahren einen Anteil von 5\% am Gesamtkollektiv haben. Die Altersstufe von 50 bis 60 Jahren macht 14\% aller operierten Patienten aus. Den weitaus größten Anteil am Gesamtkollektiv allerdings bestreiten die älteren Patienten im Alter von 60-80 Jahren mit 37\% der zwischen 60 und 70 jährigen und $36 \%$ der 70 bis 80 jährigen Patienten. 164 Patienten waren älter als 80 Jahre zum Zeitpunkt ihrer Operation, was 5\% entspricht. Die rote Kurve in Abb. 3 zeigt die zu erwartende Normalverteilung.

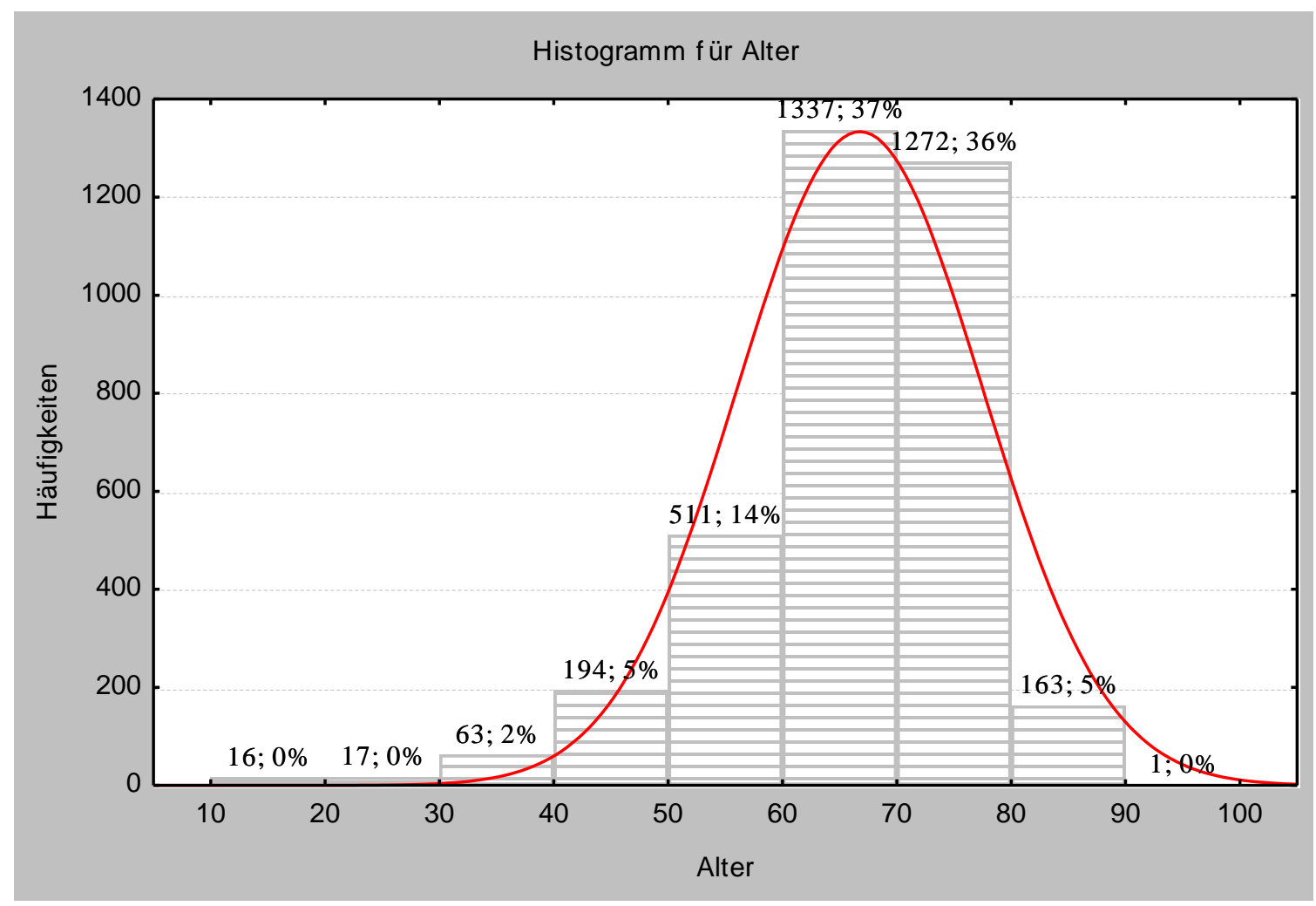

Abb. 5 Histogramm für Altersverteilung 
Der folgende Box-Plot (Abb. 6) zeigt in einer anderen graphischen Darstellungsweise, wie das Alter der Patienten verteilt ist und dass der Median bei einem Alter von 68 Jahren liegt. Das 25\%- Quantil liegt bei 61 Jahren und das 75\%- Quantil bei 74 Jahren.

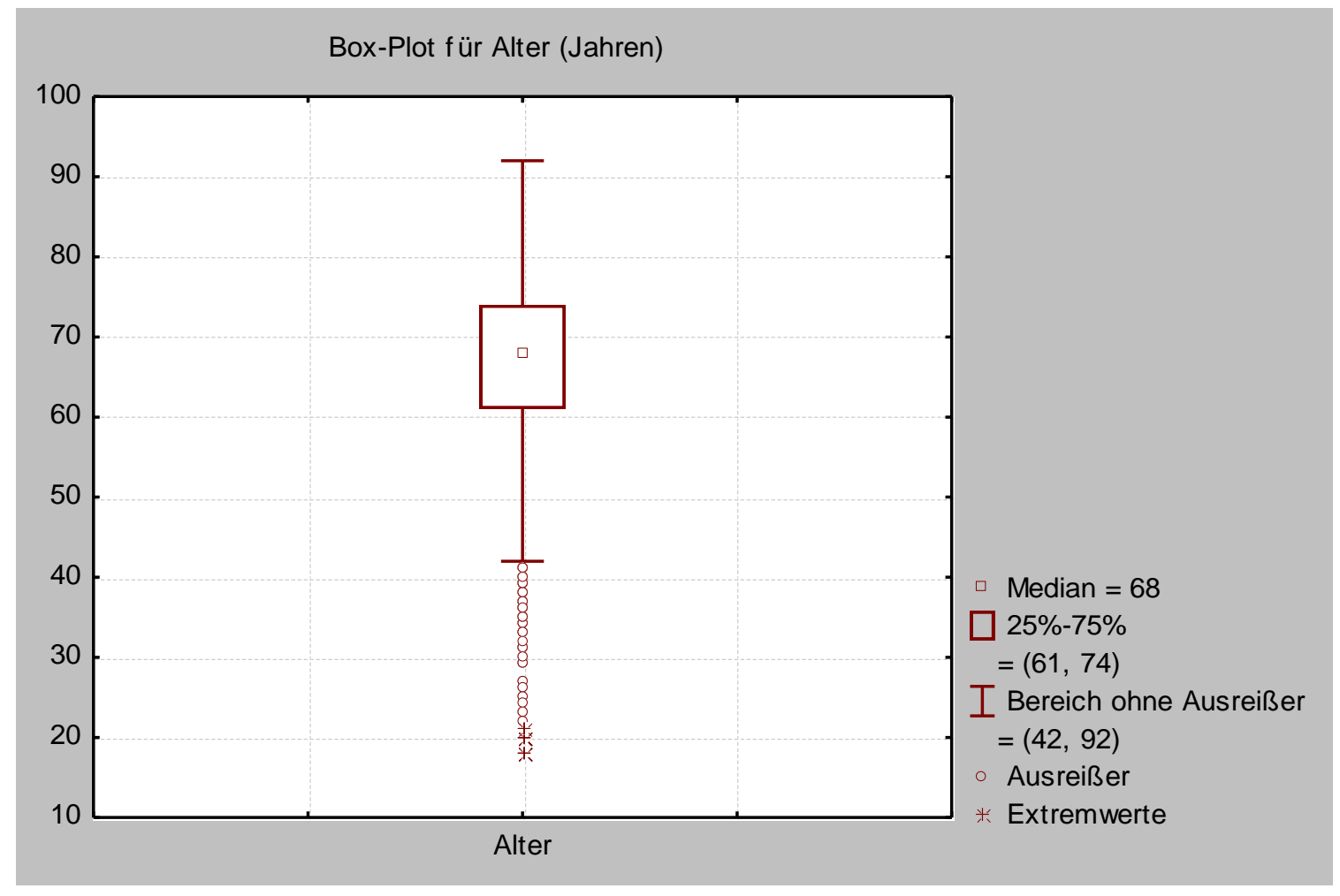

Abb. 6 Box-Plot Altersverteilung 


\subsection{Operationen}

Die 3574 operierten Patienten sind in Untergruppen je nach erfolgter Art der Operation unterteilt. 2190 Patienten erhielten ausschließlich eine Revaskularisierungsoperation und 714 Patienten wurden nur an den Herzklappen operiert. Eine Kombination dieser beiden Eingriffe erfolgte bei 537 Patienten. Die verbleibenden Operationen waren andere als die bis hier genannten, also keine Revaskularisierungs- oder Klappenoperationen. Abb. 7 veranschaulicht, dass der größte Anteil der Operationen (61\%) eine Revaskularisierung an den Koronargefäßen darstellt.

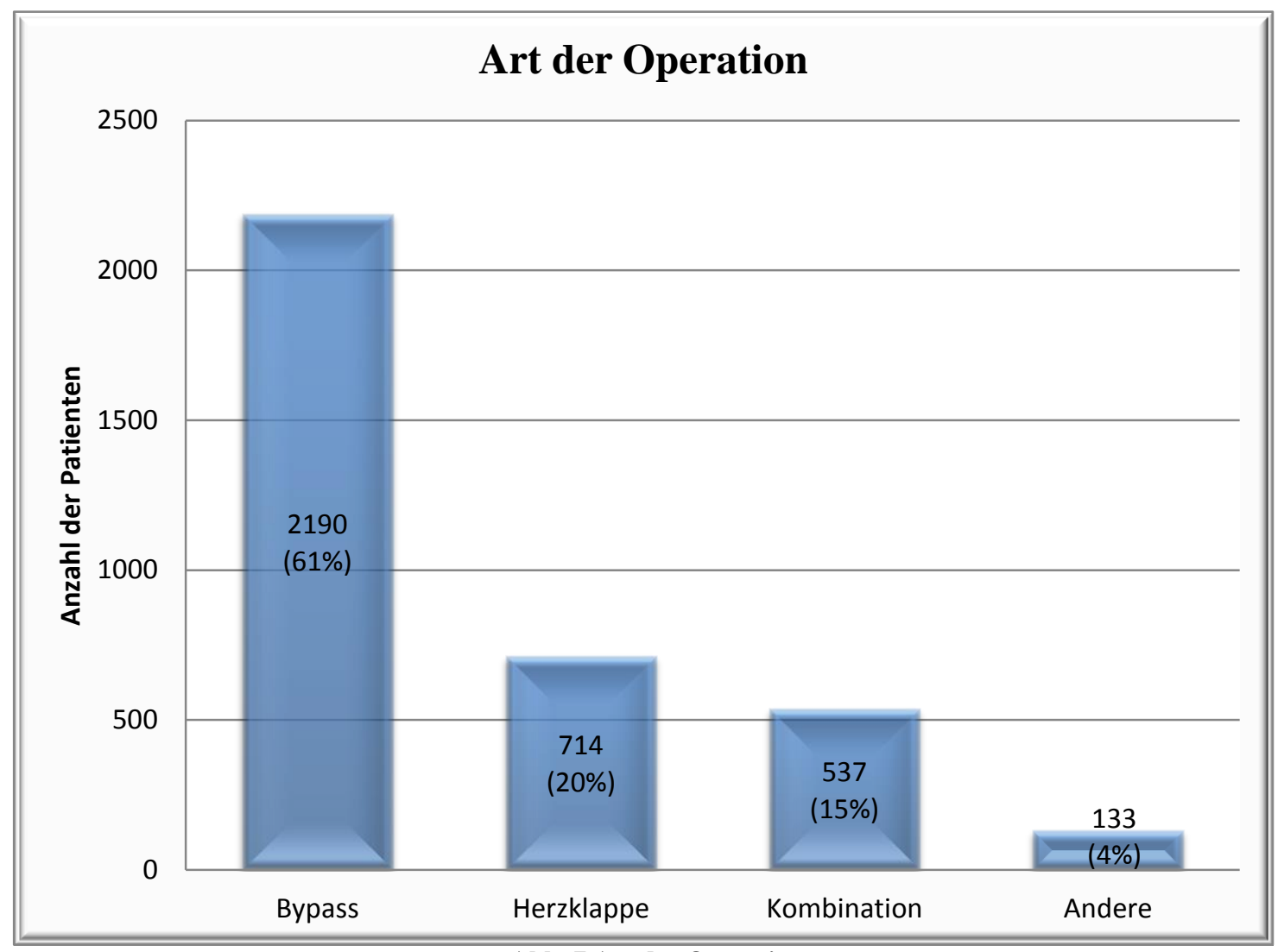

Abb. 7 Art der Operation

\subsubsection{Gesamtmortalität}

Die Mortalität bezogen auf alle 3574 operierten Patienten betrug 6,4\%, was einer absoluten Anzahl von 230 Todesfällen entspricht. 93,6\% der operierten Patienten überlebten den Eingriff am Herzen. 


\subsection{Patienten mit Einsatz eines Nierenersatzverfahrens (NEV)}

Von den 3574 operierten Patienten entwickelten 365 postoperativ ein Nierenversagen welches eine Indikation zur Nierenersatztherapie darstellte. Das heißt 10\% der am Herzen operierten Patienten bekamen ein hämofiltrationspflichtiges Nierenversagen. Die Indikationsstellung erfolgte nach den unter 3.1 genannten Leitsätzen. Als Nierenersatztherapie wurde die Hämofiltration eingesetzt. 3209 Patienten boten keine Indikation zu einem Nierenersatzverfahren. Abb. 8 zeigt den Anteil der Patienten, welche ein NEV benötigten bzw. nicht benötigten, auch prozentual.

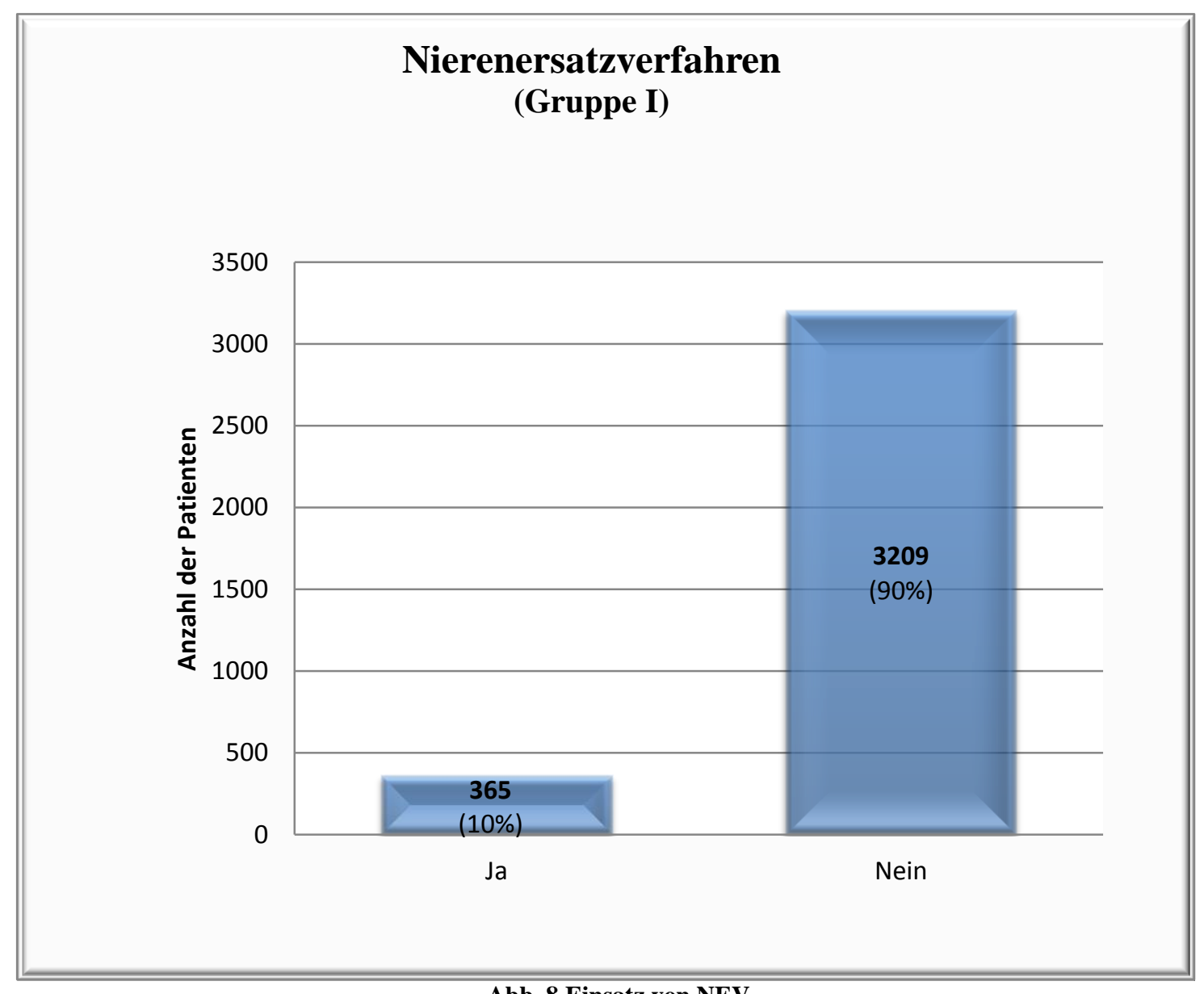

Abb. 8 Einsatz von NEV

\subsubsection{Mortalität der Patienten mit NEV}

Von den 365 Patienten, die eine Indikation zum Nierenersatzverfahren entwickelten, überlebten 188, was einem Prozentsatz von 52\% entspricht. 177 von 365 hämofiltrierten Patienten verstarben. Die Mortalität bei Patienten mit NEV liegt also bei $48 \%$. 


\subsection{Inzidenz des ANV gemäß RIFLE-Kriterien}

Die Patienten, welche nach der Definition der RIFLE-Kriterien (erhöhtes Serumkreatinin um Faktor 3 oder Abfall der GFR um 75\% oder Serumkreatininwert von 4 mg/dl) ein ANV entwickelten, werden der Gruppe II zugeordnet. Bei 251 Patienten war dies der Fall. Das heißt von 3574 operierten Patienten erfüllten 251 zumindest eines der obigen Kriterien, was einer Inzidenz des ANV in unserem Patientenkollektiv von 7\% entspricht. Abb. 9 veranschaulicht den Sachverhalt graphisch und zeigt, dass nach RIFLE-Kriterien 7\% der Patienten ein ANV entwickelten.

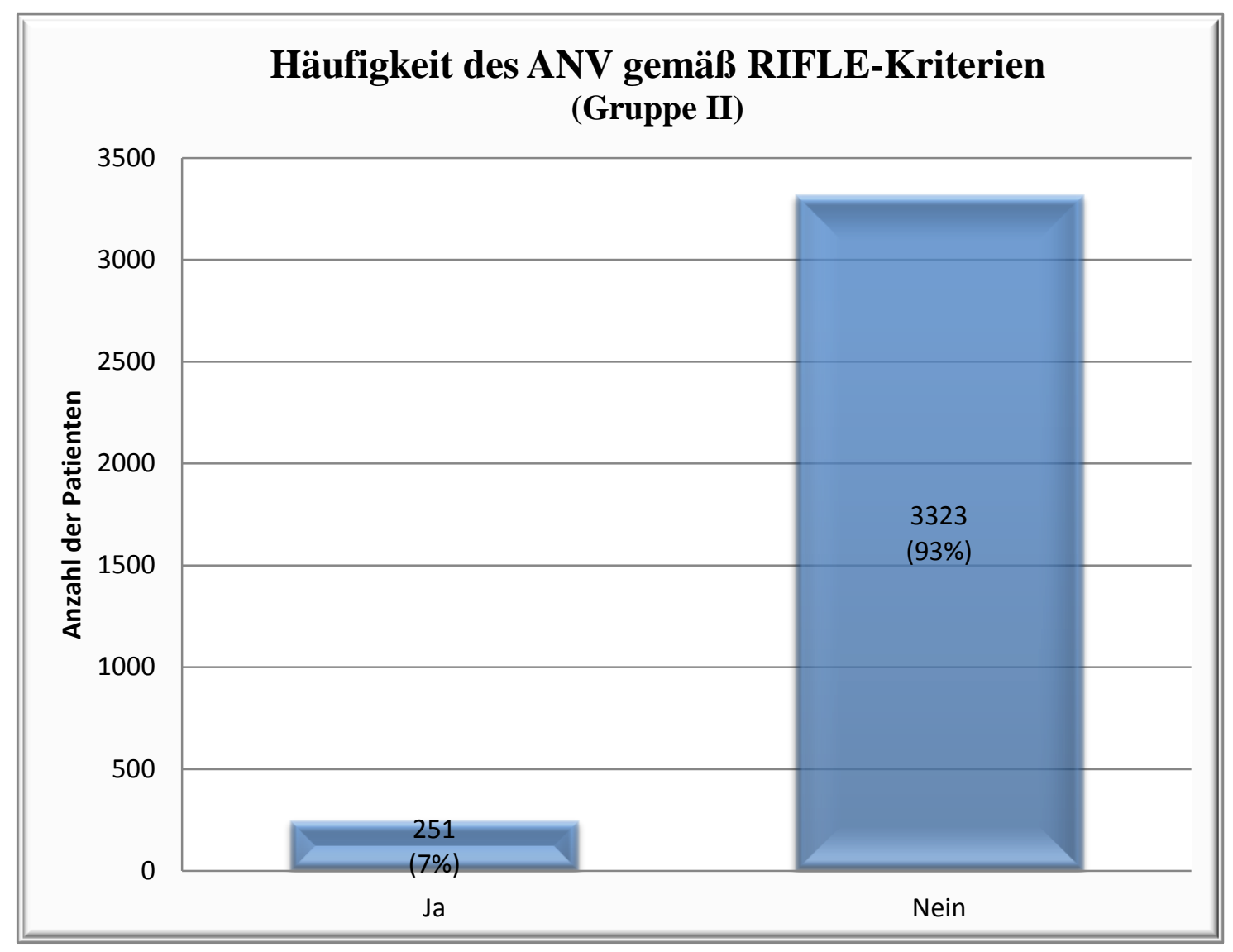

Abb. 9 Häufigkeit des ANV gemäß RIFLE-Kriterien

\subsubsection{Mortalität der Patienten mit ANV}

Von den 251 Patienten die laut den Kriterien nach RIFLE ein ANV entwickelten, verstarben

57, was $22 \%$ entspricht. $78 \%$ der Patienten überlebten das ANV, was einer absoluten Anzahl von 194 Patienten entspricht. 


\subsection{Einfluss der kategoriellen Parameter auf die Indikation eines NEV}

Im ersten Teil der Darstellung der Ergebnisse unserer statistischen Berechnungen werden die Zusammenhänge aller untersuchten Parameter und dem Bedarf eines NEV erläutert. Im zweiten Teil werden die Ergebnisse bezüglich des akuten Nierenversagens gemäß der RIFLEKriterien aufgeführt. Im dritten Teil werden alle Parameter nochmals mit dem absoluten Anstieg des Kreatinins verglichen. Die folgende Ergebnistabelle (Tab. 8) stellt der Gruppe I (ANV-H) die Patienten gegenüber, welche kein NEV benötigten. Alle kategoriellen Parameter wurden auf unterschiedliche Auswirkungen in den beiden Gruppen untersucht. Die Signifikanz ist als $\rho$-Wert angegeben und basiert auf der Berechnung mit dem $X^{2}$-Test. Ein Wert kleiner 0,05 ist als signifikant einzustufen und ein Wert unter 0,01 als sehr signifikant.

\begin{tabular}{|c|c|c|c|}
\hline \multirow[b]{2}{*}{ Parameter } & \multicolumn{2}{|c|}{ Nierenersatzverfahren } & \multirow[b]{2}{*}{$\mathrm{p}\left(\chi^{2}\right.$-Test $)$} \\
\hline & $\begin{array}{r}\text { Nein } \\
(\mathrm{n}=3209)\end{array}$ & $\begin{array}{r}\mathrm{Ja} \\
(\mathrm{n}=365)\end{array}$ & \\
\hline $\begin{array}{l}\text { Geschlecht } \\
\text { M } \\
\text { W }\end{array}$ & $\begin{array}{r}2288(91 \%) \\
920(88 \%)\end{array}$ & $\begin{array}{r}234(9 \%) \\
131(12 \%)\end{array}$ & $<0.01$ \\
\hline $\begin{array}{l}\text { Bypass } \\
\text { Ja } \\
\text { Nein }\end{array}$ & $\begin{array}{r}2451(90 \%) \\
758(89 \%)\end{array}$ & $\begin{array}{r}276(10 \%) \\
89(11 \%)\end{array}$ & 0.80 \\
\hline $\begin{array}{l}\text { Klappen } \\
\text { Ja } \\
\text { Nein }\end{array}$ & $\begin{array}{l}1090(87 \%) \\
2119(91 \%)\end{array}$ & $\begin{array}{r}161(13 \%) \\
204(9 \%)\end{array}$ & $<0.01$ \\
\hline $\begin{array}{l}\text { Re-Op } \\
\text { Ja } \\
\text { Nein }\end{array}$ & $\begin{array}{r}145(71 \%) \\
3064(91 \%)\end{array}$ & $\begin{array}{l}58(29 \%) \\
307(9 \%)\end{array}$ & $<0.01$ \\
\hline $\begin{array}{l}\text { IABP } \\
\text { Ja } \\
\text { Nein }\end{array}$ & $\begin{array}{r}58(59 \%) \\
3151(91 \%)\end{array}$ & $\begin{array}{l}40(41 \%) \\
325(9 \%)\end{array}$ & $<0.01$ \\
\hline $\begin{array}{l}\text { Pumpe } \\
\text { Zentrifugal } \\
\text { Roller }\end{array}$ & $\begin{array}{r}2687(90 \%) \\
437(91 \%)\end{array}$ & $\begin{array}{r}312(10 \%) \\
49(9 \%)\end{array}$ & 0.89 \\
\hline $\begin{array}{l}\text { Kardioplegie } \\
\text { Bretschneider } \\
\text { Blutkardioplegie }\end{array}$ & $\begin{array}{l}1434(91 \%) \\
1689(90 \%)\end{array}$ & $\begin{array}{r}144(9 \%) \\
198(10 \%)\end{array}$ & 0.20 \\
\hline $\begin{array}{l}\text { Temperatur } \\
\text { Normothermie } \\
\text { MildeHypothermie } \\
\text { TiefeHypothermie }\end{array}$ & $\begin{array}{r}1789(92 \%) \\
693(87 \%) \\
14(74 \%)\end{array}$ & $\begin{array}{r}163(8 \%) \\
100(13 \%) \\
5(26 \%)\end{array}$ & $<0.01$ \\
\hline $\begin{array}{l}\text { Operationen } \\
\text { Andere } \\
\text { Nur Bypass } \\
\text { Nur Herzklappen } \\
\text { Kombination }\end{array}$ & $\begin{array}{r}113(85 \%) \\
2006(92 \%) \\
645(90 \%) \\
445(83 \%)\end{array}$ & $\begin{array}{l}20(15 \%) \\
184(8 \%) \\
69(10 \%) \\
92(17 \%)\end{array}$ & $<0.01$ \\
\hline
\end{tabular}


Aus den Berechnungen geht hervor, dass zwischen der Gruppe I (ANV-H) und allen anderen Patienten deutliche, teils signifikante Unterschiede auftreten. Von 1051 operierten Frauen haben 131 eine Indikation zum NEV dargestellt, was 12\% entspricht. Dagegen haben von 2522 operierten Männern 234 ein NEV bekommen, was 9\% entspricht. Dieser prozentuale Unterschied mag zahlenmäßig gering erscheinen, ist aber auf die große Stichprobe bezogen mit $\rho<0,01$ ein hoch signifikanter Unterschied. In der graphischen Darstellung (Abb. 10) wird deutlich, dass, bezogen auf den im Vergleich zu den Männern kleinen Anteil operierter Frauen, ein relativ hoher prozentualer Anteil an Nierenersatzverfahren kommt.

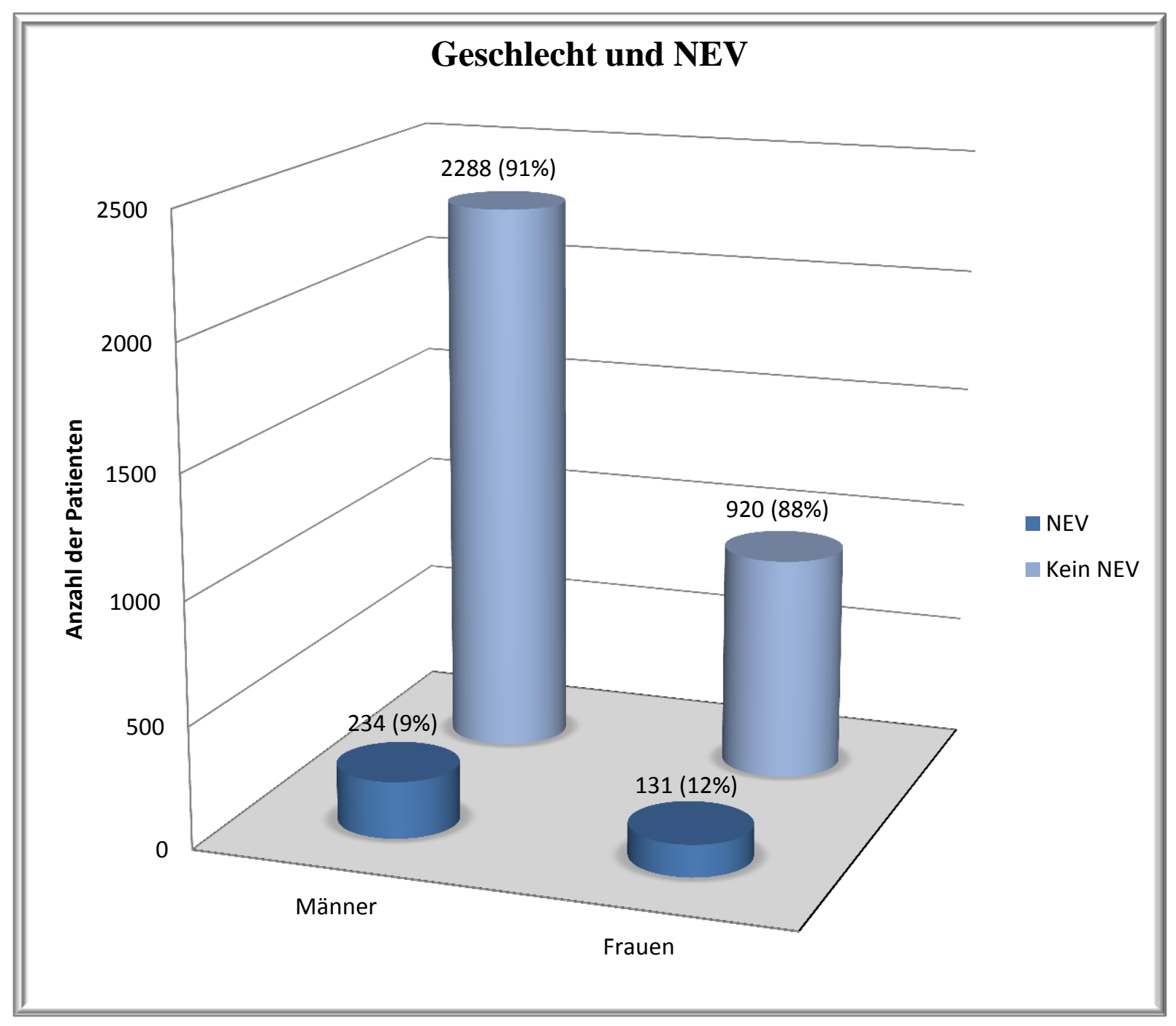

Abb.10 Geschlecht und NEV

Eine Bypassoperation an sich ist kein eigener Risikofaktor für die Entwicklung eines ANV. Der $\rho$-Wert beträgt hier 0,8 und legt nahe, dass es keinen signifikanten Unterschied zwischen den Patienten mit und ohne Bypass-Operation gibt. Im Gegensatz dazu ist eine Klappenoperation, gleich ob mit oder ohne zusätzlicher Prozedur, ein Risikofaktor für die Notwendigkeit eines NEV. Die prozentuale Verteilung von 13\% NEV bei Patienten mit 
Klappenoperation versus 9\% NEV ohne Klappenoperation weist auf einen Unterschied in beiden Gruppen hin. Der $\rho$-Wert liegt unterhalb von 0,01. In Abb. 11 ist auch optisch deutlich zu erkennen, dass der Anteil von Patienten mit NEV bei Klappenoperationen im Verhältnis zu allen anderen Prozeduren deutlich höher liegt.

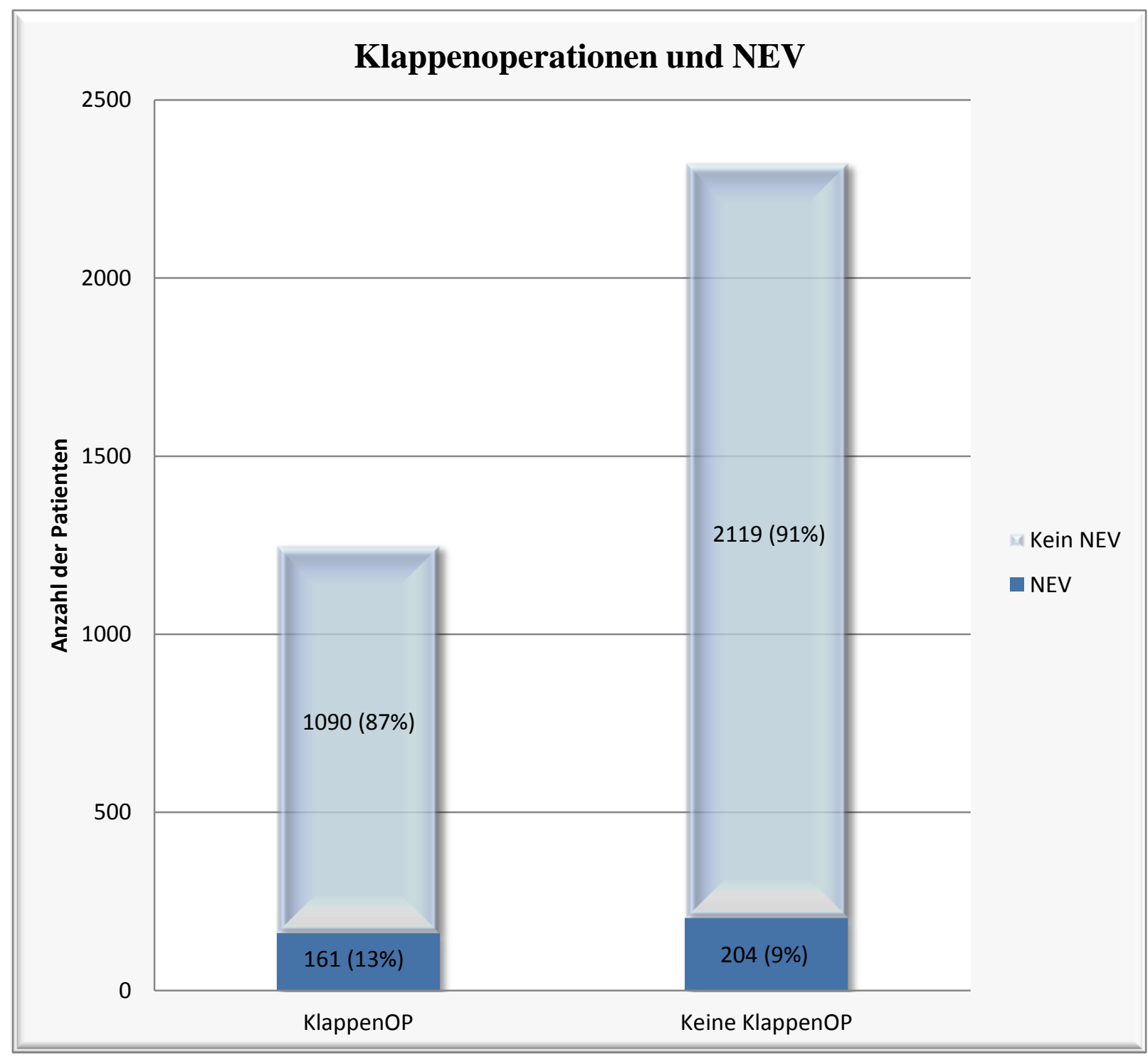

Abb.11 Klappenoperationen und NEV

Um die einzelnen Operationen unter sich nochmals differenzieren zu können, wurde eine weitere Analyse vorgenommen. Unter dem Parameter „Operationen“ sind nur Patienten gezählt worden, die ausschließlich eine Bypassoperation oder ausschließlich eine Klappenoperation bzw. eine Kombination beider, oder eine andere Operation erhielten. Auch hier sind die Unterschiede mit $\rho<0,01$ als signifikant zu werten. Die Kombinationsoperationen haben mit $17 \%$ am häufigsten ein NEV nach sich gezogen. Die 
Operationen an den Herzklappen unterscheiden sich mit 10\% deutlich von den Kombinationseingriffen, aber auch den reinen Bypassoperationen mit 8\%. Die anderen Operationen haben mit 15\% auch eine häufige Indikation zum NEV geboten. In Absteigender und jeweils signifikanter Reihenfolge sind die Operationen also wie folgt zu bewerten: Kombination von Bypass und KlappenOP (17\%) > Andere Operationen (15\%)> HerzklappenOP (10\%) > Bypassoperation (8\%). Wenn man, wie in Abb. 12 die jeweilige Gruppe einer Art von Operation als 100\% darstellt, wird der prozentual-unterschiedliche Anteil zwischen den Gruppen gut sichtbar.

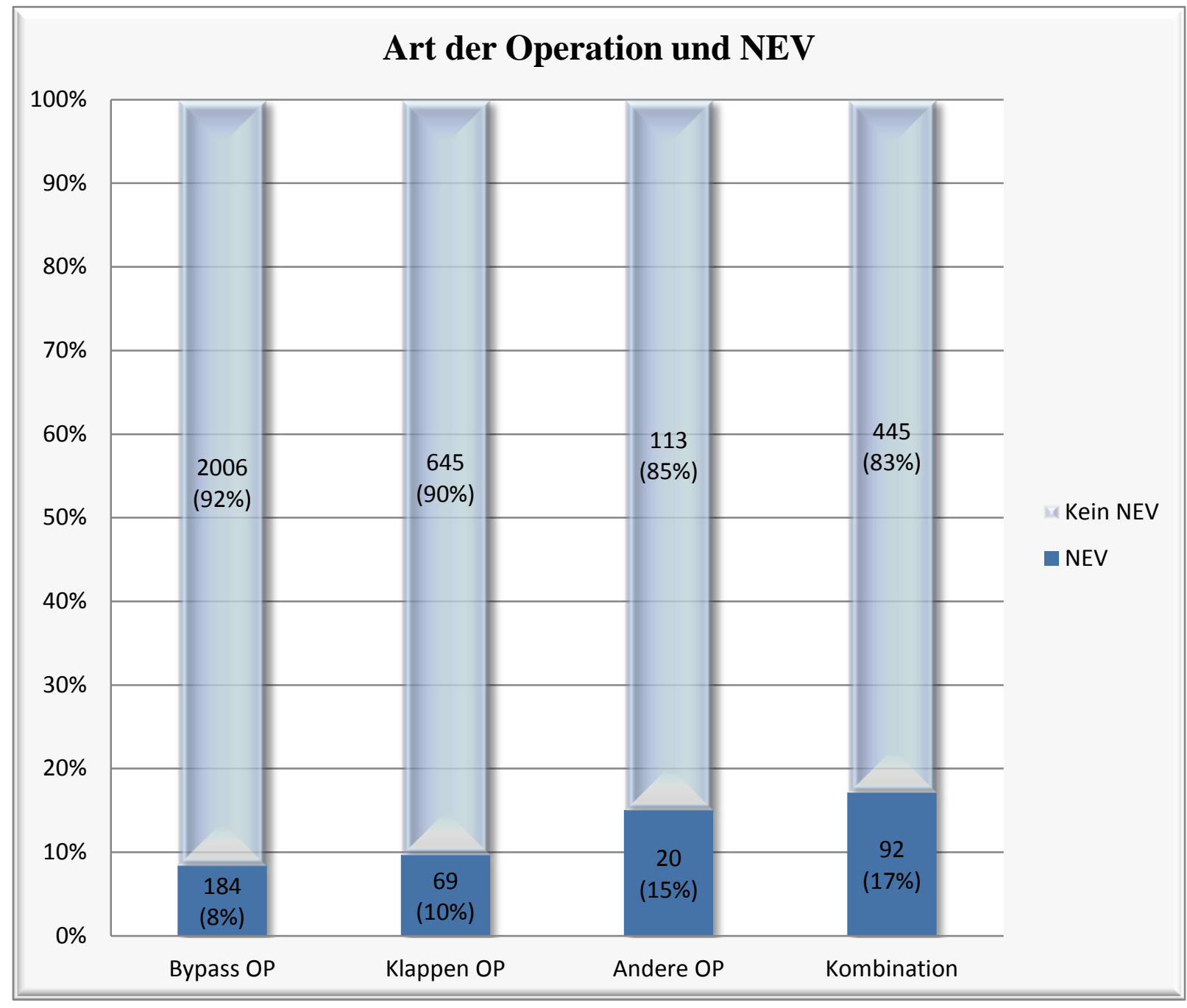

Abb.12 Art der Operation und NEV

Das Ereignis einer Re-Operation und der Einsatz einer IABP waren hoch signifikant mit dem Einsatz eines NEV verbunden. Im Vergleich zu Erstoperationen, welche in $9 \%$ zu dem Einsatz eines NEV führten, waren Reoperationen mit 29\% öfter mit einem NEV assoziiert. Die Intraaortale Ballonpumpe als Zeichen einer kardialen Pumpschwäche führte in $41 \%$ der 
Fälle zu einem NEV. Wurde diese nicht benötigt kam auch das NEV nur in 9\% zum Einsatz. Die Wahl der Pumpe in der Herz-Lungen-Maschine und die Wahl der Kardioplegielösung haben in Bezug auf den Bedarf eines NEV keine signifikante Auswirkung allerdings wird bei den nachfolgenden Untersuchungen deutlich werden, dass hier, zumindest hinsichtlich eines Anstiegs des Serumkreatinins, signifikante Unterschiede bestehen.

Als weiterer Parameter wurde die Temperatur während der Operation an der HLM untersucht. Es kann aus der Berechnung ein hoch signifikanter Zusammenhang zwischen der Temperatur und dem Einsatz eines NEV mit $\rho<0,01$ hergestellt werden. Die 1789 Patienten mit einer Normothermie $\left(37-34^{\circ} \mathrm{C}\right)$ benötigten nur mit $8 \%$ ein NEV, wohingegen die 693 Patienten mit einer milden Hypothermie $\left(33-28^{\circ} \mathrm{C}\right)$ mit $13 \%$ ein NEV benötigten. Die 14 Patienten, die sich in tiefer Hypothermie $\left(27-18^{\circ} \mathrm{C}\right)$ befanden, bekamen sogar zu $26 \%$ eine Indikation zum NEV. Dieser Zusammenhang ist in Abb.13 zur besseren Veranschaulichung als Anteil der $100 \%$ innerhalb der jeweiligen Gruppe dargestellt.

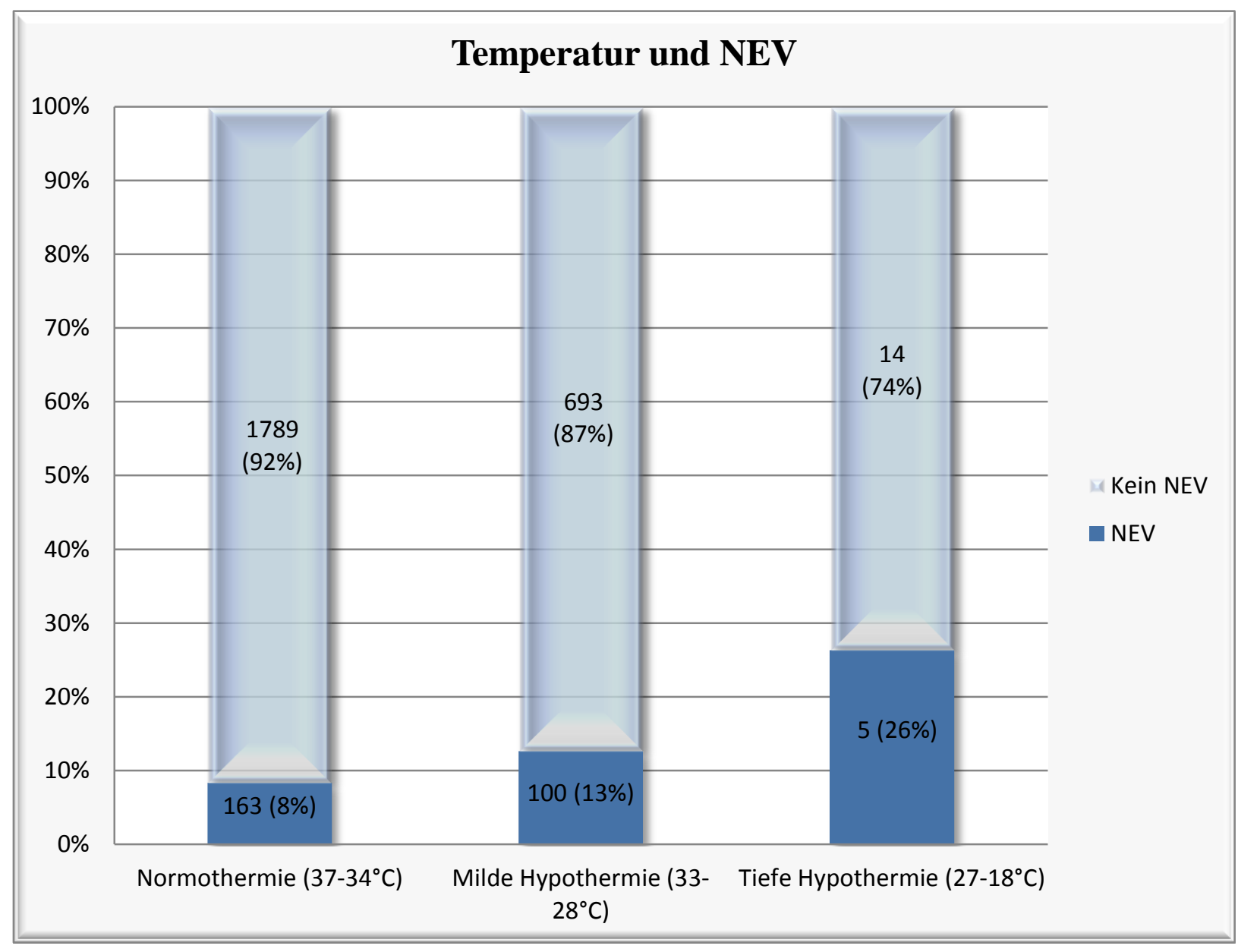

Abb.13 Temperatur und NEV 


\subsection{Einfluss der metrischen Parameter auf die Indikation eines NEV}

Die folgende Ergebnistabelle (Tab.9) zeigt den Einfluss der metrischen Parameter auf die Indikation eines NEV. Zu jedem untersuchten Parameter sind die jeweiligen Werte des 25\%-, 50\%- und 75\%- Quantils angegeben. Der p-Wert nach der Berechnung im Wilcoxon-MannWhitney-Test ist hinsichtlich aller Parameter kleiner als 0,01 und damit hoch signifikant. Wenn man die Quantilenwerte zwischen den Gruppen mit und ohne Nierenersatzverfahren vergleicht, werden die Unterschiede deutlich. In den folgenden Box-Plots werden die einzelnen Parameter genauer beschrieben und differenziert.

\begin{tabular}{|c|c|c|c|}
\hline \multirow{2}{*}{ Parameter } & \multicolumn{2}{|c|}{$\begin{array}{c}\text { Nierenersatzverfahren } \\
(25 \%-, 50 \% \text { - und } 75 \% \text {-Quantil) }\end{array}$} & \multirow{2}{*}{$\mathrm{p}$ (Wilcoxon) } \\
\hline & $\begin{array}{r}\text { Nein } \\
(\mathrm{n}=3209)\end{array}$ & $\begin{array}{r}\mathrm{Ja} \\
(\mathrm{n}=365)\end{array}$ & \\
\hline Alter (Jahre) & $61.0,68.0,74.0$ & $66.0,71.0,76.0$ & $<0.01$ \\
\hline Kreatinin A (mg/dl) & $0.8,0.9,1.1$ & $1.1,1.5,2.0$ & $<0.01$ \\
\hline Kreatinin B (mg/dl) & $0.9,1.1,1.4$ & $2.4,3.1,4.0$ & $<0.01$ \\
\hline Operationsdauer(min) & $227.0,270.0,325.0$ & $275.0,335.0,425.5$ & $<0.01$ \\
\hline Ischämiezeit (min) & $60.0,78.0,101.0$ & $69.0,94.0,128.0$ & $<0.01$ \\
\hline Perfusionszeit (min) & $97.0,124.0,156.0$ & $124.0,168.0,220.0$ & $<0.01$ \\
\hline Reperfusionszeit(min) & $27.0,37.0,48.0$ & $36.0,50.0,74.0$ & $<0.01$ \\
\hline Min.Temperatur $\left({ }^{\circ} \mathrm{C}\right)$ & $33.1,34.4,35.9$ & $33.1,34.1,35.8$ & $<0.01$ \\
\hline
\end{tabular}

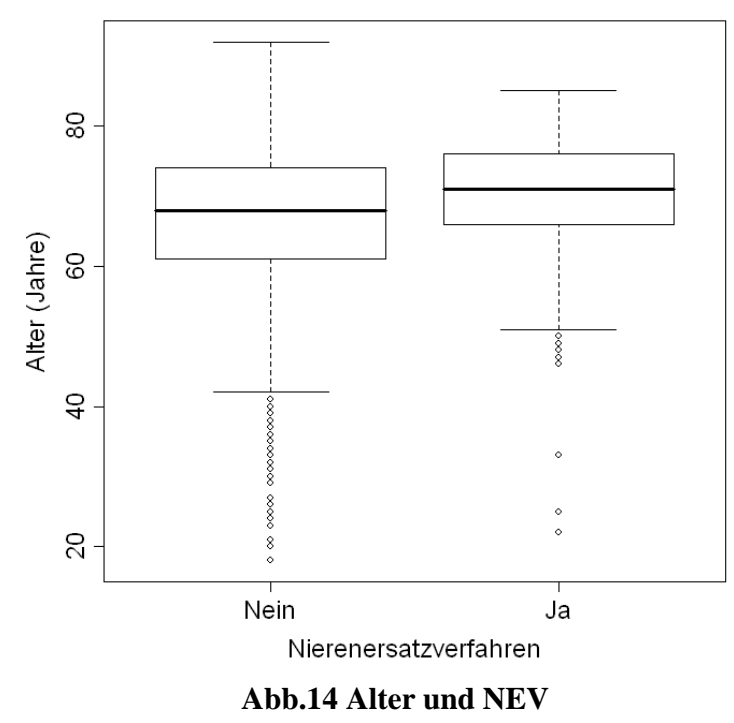

die zweite $24 \mathrm{~h}$ später (B). Beide Werte zeigen in den Gruppen mit und ohne NEV signifikante Unterschiede, was bei dem Serumkreatinin als Marker für die Nierenfunktion auch zu
Die Patienten, bei denen ein NEV indiziert war, waren signifikant älter als die Patienten, welche keine solche Therapiemaßnahme benötigten. (s. Abb. 14) Das 50\%- Quantil der Patienten ohne NEV liegt bei 68,0 Jahren, wohingegen das der Patienten mit NEV bei 71,0 Jahren liegt. Die Werte für das 25\%- und 75\%- Quantil unterscheiden sich ebenfalls signifikant. Die Kreatininwerte A und B beziehen sich auf zwei Blutabnahmen, eine direkt nach der Operation nach Ankunft auf der Intensivstation (A) und 
erwarten ist. Es sei aber darauf hingewiesen, dass der Serumkreatininwert nicht in die Entscheidungsfindung zur Hämofiltration mit eingeht. Dennoch zeigen die in Tab.9 stehenden Werte deutliche Unterschiede zwischen dem 50\%- Quantil von 0,9 mg/dl ohne NEV im Vergleich zu 1,5 mg/dl mit NEV beim Kreatininwert A. Beim Kreatininwert B liegt das 50\%Quantil bei 1,1 mg/dl ohne NEV und bei 3,1 mg/dl mit NEV.

Die Dauer der gesamten Operation aber auch die einzelnen Zeitabschnitte der Ischämie, Perfusion und Reperfusion waren jeweils hochsignifikant mit einem Einsatz eines NEV assoziiert. Die Patienten, bei denen ein NEV indiziert war, hatten signifikant längere Operationsdauern. In der Abb.15 ist in Form eines Box-Plots dargestellt wie sich die beiden Gruppen unterscheiden. Bei den Patienten ohne NEV lag das 25\%- Quantil bei 227 min, das 50\%- Quantil bei 270 min und das 75\%- Quantil bei $325 \mathrm{~min}$. Demgegenüber waren die Werte bei der Gruppe mit NEV mit 275 min beim 25\%-Quantil, 335 min beim 50\%-Quantil und 425 min beim 75\%- Quantil deutlich höher. Bei der Ischämiezeit sind die Unterschiede ebenfalls hoch signifikant (Abb.16).

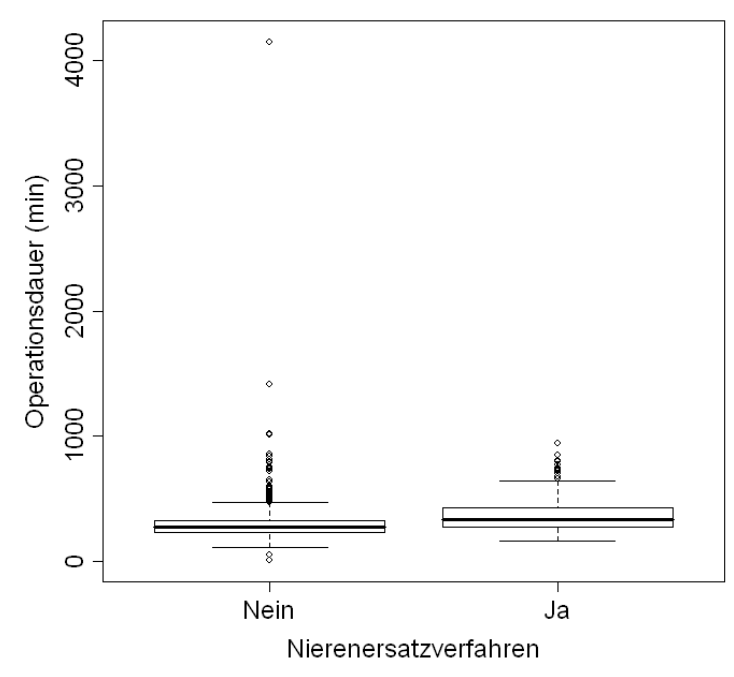

Abb.15 Operationsdauer und NEV

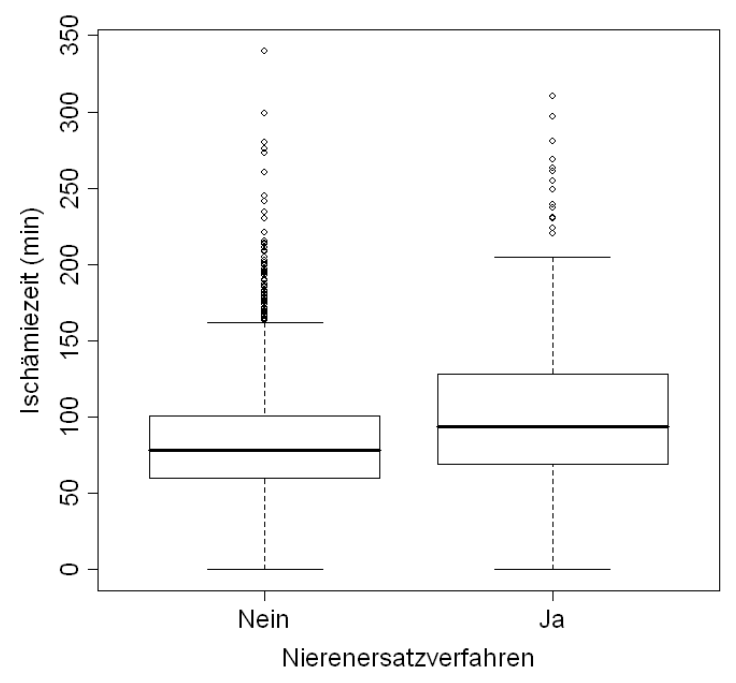

Abb.16 Ischämiezeit und NEV

Ohne NEV liegt das 50\%- Quantil bei 78 min und mit NEV bei 94 min. Bei den Patienten mit NEV waren die Ischämiezeiten also signifikant länger. Der gleiche Effekt ist bei der Perfusionszeit (Abb. 17) und der Reperfusionszeit (Abb.18) zu beobachten. Hierbei betragen die 50\%- Quantile 124 bzw. 37 min ohne NEV und 168 bzw. 50 min mit NEV. Alle Werte sind in Tab.9 aufgeführt. 


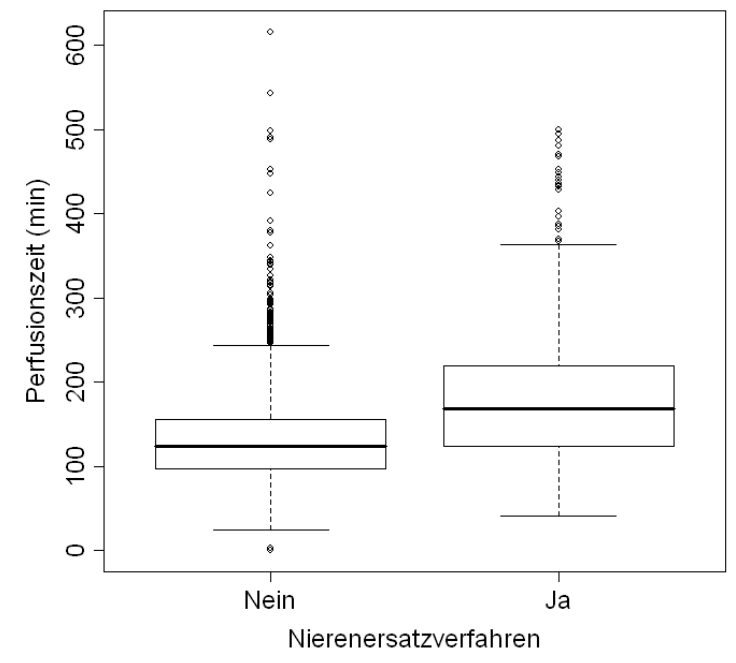

Abb.17 Perfusionszeit und NEV

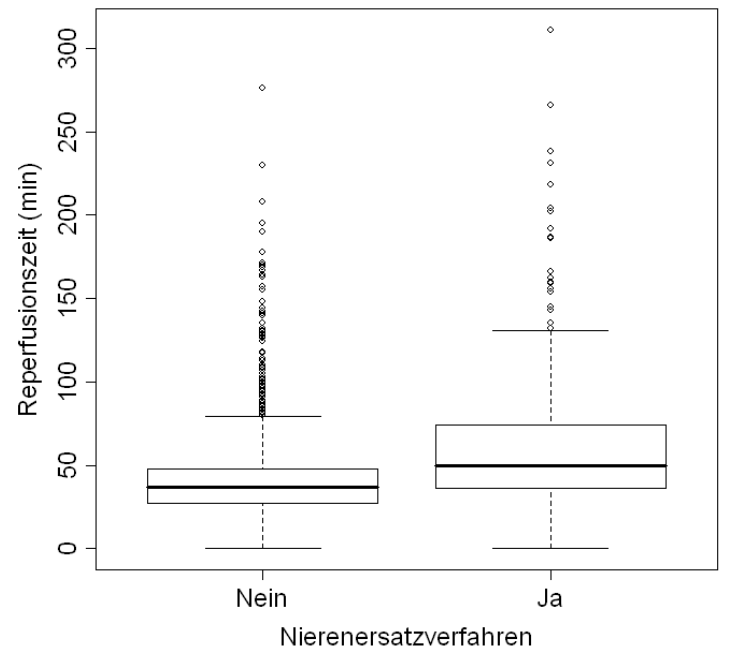

Abb.18 Reperfusionszeit und NEV

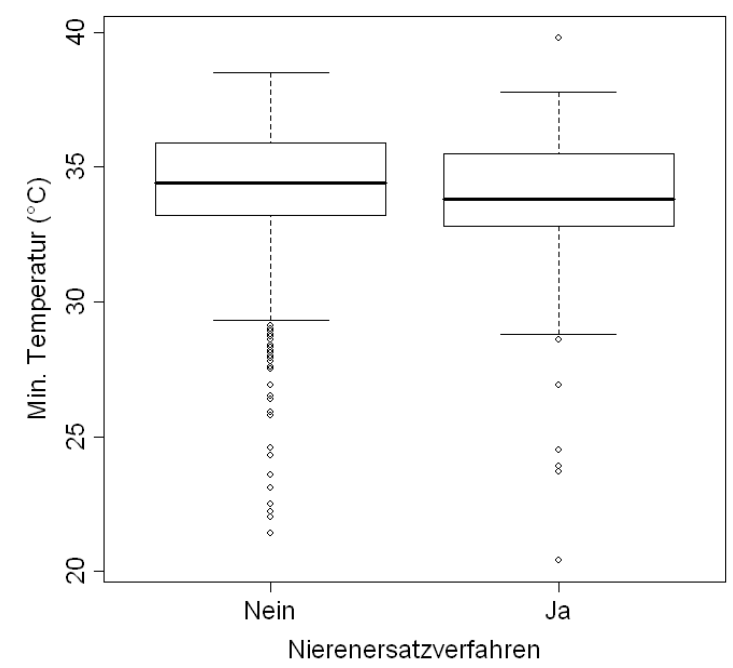

Abb.19 niedrigste Temperatur und NEV
Auch im Bezug auf die minimale Temperatur während der Operation ergeben sich zwischen den Patienten, die ein NEV benötigten und denen, die keines benötigten signifikante Unterschiede. Das 50\%- Quantil liegt in der ersten Gruppe bei 34,4 Grad Celsius und der zweiten Gruppe bei 34,1 Grad Celsius. Das heißt Patienten, die postoperativ ein ANV entwickelten, waren während der Operation „kälter“. Der neben-stehende Box-Plot (Abb.19) demonstriert graphisch den Unterschied. 


\subsection{Blutverlust und NEV}

Bei der Untersuchung des Zusammenhangs zwischen dem Einsatz von NEV und postoperativen Blutverlusten wurde nicht nur die absolute Menge an verlorenem Blut, sondern auch die Dauer und der zeitliche Verlauf des Blutverlustes über die ersten 6 postoperativen Tage einbezogen. Es handelt sich also um eine longitudinale Analyse, welche einen Gruppeneffekt, einen Zeiteffekt und die Wechselwirkung des Zeiteffekts der beiden Gruppen beurteilt. Es stellte sich heraus, dass bei Patienten, bei denen ein NEV indiziert war, neben der absoluten Menge an Blutverlusten auch der zeitliche Verlauf des Blutverlustes signifikant erhöht bzw. verlängert war. Ebenfalls signifikant war die Wechselwirkung zwischen den Zeitverläufen der beiden Gruppen mit und ohne NEV. Die unten stehende Abb. 20 zeigt diese Effekte anschaulich anhand der Mediane, d.h. der 50\%- Quantile des Blutverlustes in $\mathrm{ml}$, welcher über die 6 Tage aufgetragen ist.

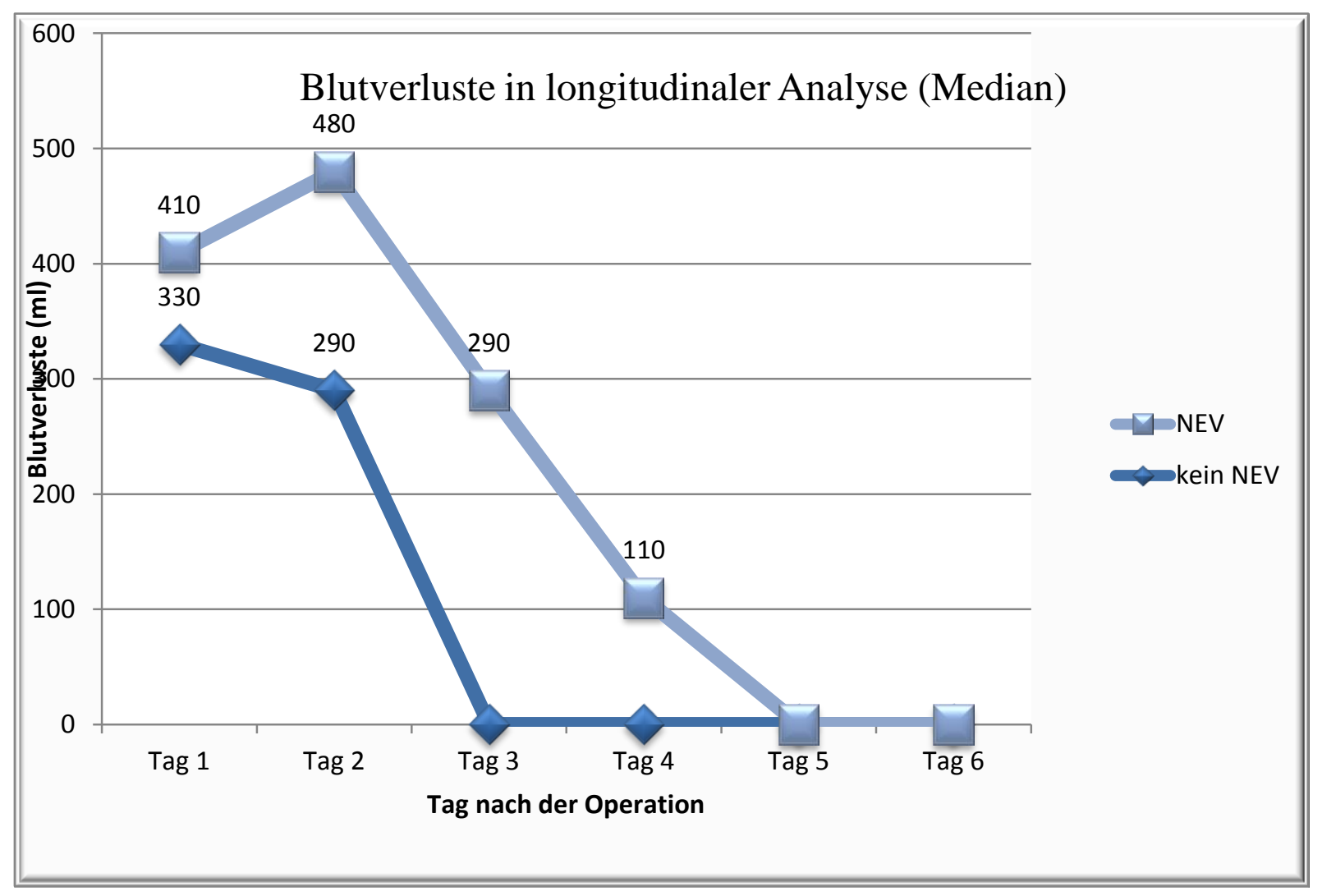

Abb.20 Blutverluste über die Zeit (Median)

Der Gruppeneffekt zeigt sich durch das unterschiedliche Niveau der beiden Kurven bis zum

Tag 5, ab dem sie dann beide auf den Wert 0 abgefallen sind. Der Zeiteffekt ist die

Veränderung des Blutverlustes über die Zeit hinweg. Aus der Abbildung wird ersichtlich, dass 
ab dem zweiten Tag in beiden Gruppen der Blutverlust stetig abnimmt. In der Gruppe mit NEV steigt er hingegen am zweiten Tag auf seinen Höchstwert. Die Wechselwirkung beschreibt den unterschiedlichen Zeitverlauf zwischen den beiden Gruppen. Alle diese Parameter (Gruppe, Zeit und Wechselwirkung) sind mit $\mathrm{p}<0,01$ deutlich signifikant.

Im 75\%- Quantil weichen die Werte noch stärker auseinander, wie Abb.21 veranschaulicht. Die einzelnen Werte für alle Quantile sind in Tab.10 aufgeführt. Es wird deutlich, dass das 25\%- Quantil über alle 6 Tage hinweg 0 ist, d.h., dass die ersten 25\% Prozent der Werte an allen 6 Tagen immer noch bei 0 lagen. Ab dem 2. postoperativen Tag gingen die Werte für das 50\%- Quantil für die Patienten ohne NEV auf 0. Für das 75\%- Quantil einen Tag später, am 3. postoperativen Tag. Für die Gruppe mit NEV stellte sich der Blutverlust im Median am 4. postoperativen Tag ein und für das 75\%- Quantil am 6. postoperativen Tag.

\begin{tabular}{|c|c|c|c|c|}
\hline Tag & NEV & $25 \%$-Quantil & $\begin{array}{l}\text { 50\%-Quantil } \\
\text { (Median) }\end{array}$ & $75 \%$-Quantil \\
\hline 0 & Nein & 0 & 330 & 590 \\
\hline 0 & $\mathrm{Ja}$ & 0 & 410 & 970 \\
\hline 1 & $\begin{array}{c}\text { Nein } \\
\mathrm{Ja}\end{array}$ & $\begin{array}{l}0 \\
0\end{array}$ & $\begin{array}{l}290 \\
480\end{array}$ & $\begin{array}{c}490 \\
1020\end{array}$ \\
\hline 2 & $\begin{array}{c}\text { Nein } \\
\mathrm{Ja}\end{array}$ & $\begin{array}{l}0 \\
0\end{array}$ & $\begin{array}{c}0 \\
290\end{array}$ & $\begin{array}{l}180 \\
560\end{array}$ \\
\hline 3 & $\begin{array}{c}\text { Nein } \\
\text { Ja }\end{array}$ & $\begin{array}{l}0 \\
0\end{array}$ & $\begin{array}{c}0 \\
110\end{array}$ & $\begin{array}{c}0 \\
320\end{array}$ \\
\hline 4 & $\begin{array}{c}\text { Nein } \\
\text { Ja }\end{array}$ & $\begin{array}{l}0 \\
0 \\
\end{array}$ & $\begin{array}{l}0 \\
0\end{array}$ & $\begin{array}{c}0 \\
230\end{array}$ \\
\hline 5 & $\begin{array}{c}\text { Nein } \\
\mathrm{Ja}\end{array}$ & $\begin{array}{l}0 \\
0\end{array}$ & $\begin{array}{l}0 \\
0\end{array}$ & $\begin{array}{c}0 \\
100\end{array}$ \\
\hline 6 & $\begin{array}{c}\text { Nein } \\
\text { Ja }\end{array}$ & $\begin{array}{l}0 \\
0\end{array}$ & $\begin{array}{l}0 \\
0\end{array}$ & $\begin{array}{l}0 \\
0\end{array}$ \\
\hline
\end{tabular}

Tab.10 Blutverluste über die Zeit 
Abbildung 21 stellt nochmals graphisch die verschiedenen Kurvenverläufe zwischen allen Gruppen dar. Die rote Kurve zeigt die Werte des 75\%- Quantils für die Patienten mit NEV im Vergleich zur gelben Kurve, welche die Gruppe ohne NEV beschreibt. Die grüne und blaue Kurve sind die Darstellung der Werte für den Median in beiden Gruppen, (vergleiche Abb. 20) und sind hier zum Vergleich nochmals mit aufgenommen.

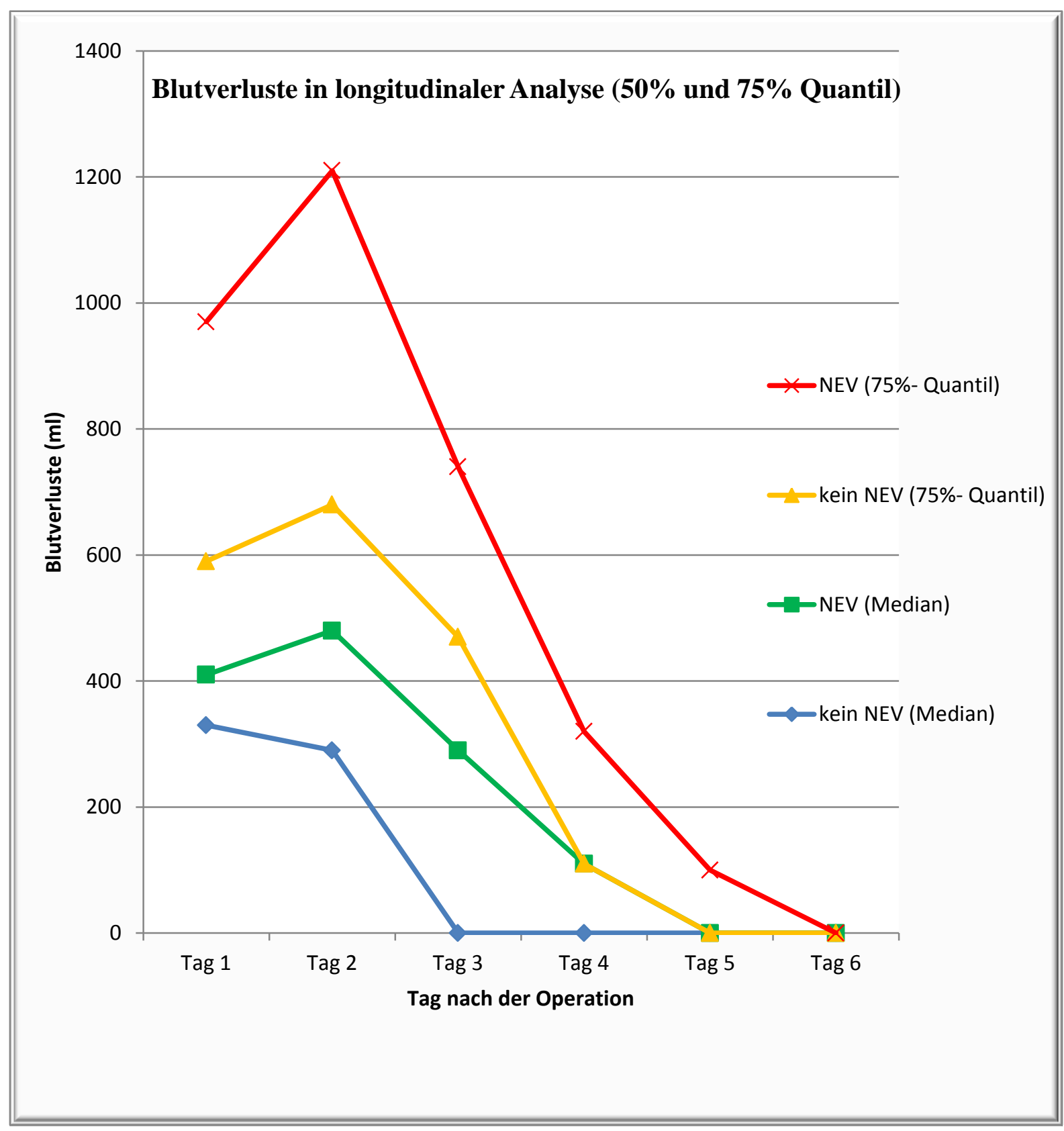

Abb.21 Blutverluste (50\%- \& 75\%- Quantil) 


\subsection{Einfluss der kategoriellen Parameter auf das Auftreten eines ANV gemäß RIFLE}

In diesem zweiten Teil der Ergebnisdarstellung werden die Assoziationen zwischen den zu untersuchenden Variablen und dem Auftreten eines ANV gemäß der Definition der RIFLEKriterien aufgeführt. In Tab.11 sind alle Ergebnisse in absoluten Zahlenwerten und Prozent aufgeführt. Deutlich wird, dass auch hier signifikante Unterschiede zwischen der Gruppe II (ANV) und allen anderen Patienten festzustellen sind. In hoch signifikanter Weise betrifft dies die Reoperationen, den Einsatz einer IABP, die Temperatur und die Art der Operation.

\begin{tabular}{|c|c|c|c|}
\hline \multirow[b]{2}{*}{ Parameter } & \multicolumn{2}{|c|}{ ANV gemäß RIFLE } & \multirow[b]{2}{*}{$\mathrm{p}\left(\chi^{2}\right.$-Test $)$} \\
\hline & $\begin{array}{c}\text { Nein } \\
(\mathrm{n}=3323)\end{array}$ & $\begin{array}{c}\text { Ja (GruppeII) } \\
(\mathrm{n}=251)\end{array}$ & \\
\hline $\begin{array}{l}\text { Geschlecht } \\
\text { M } \\
\text { W } \\
\end{array}$ & $\begin{array}{r}2341(70 \%) \\
981(30 \%) \\
\end{array}$ & $\begin{array}{r}181(72 \%) \\
70(28 \%) \\
\end{array}$ & 0.62 \\
\hline $\begin{array}{l}\text { Bypass } \\
\text { Ja } \\
\text { Nein }\end{array}$ & $\begin{array}{r}2520(76 \%) \\
803(24 \%)\end{array}$ & $\begin{array}{r}207(82 \%) \\
44(18 \%)\end{array}$ & 0.02 \\
\hline $\begin{array}{l}\text { Klappen } \\
\text { Ja } \\
\text { Nein }\end{array}$ & $\begin{array}{l}1157(35 \%) \\
2166(65 \%)\end{array}$ & $\begin{array}{r}94(37 \%) \\
157(63 \%)\end{array}$ & 0.10 \\
\hline $\begin{array}{l}\text { Re-Op } \\
\text { Ja } \\
\text { Nein }\end{array}$ & $\begin{array}{r}172(5 \%) \\
3151(95 \%)\end{array}$ & $\begin{array}{r}31(12 \%) \\
220(88 \%)\end{array}$ & $<0.01$ \\
\hline $\begin{array}{l}\text { IABP } \\
\text { Ja } \\
\text { Nein }\end{array}$ & $\begin{array}{r}83(2 \%) \\
3240(98 \%)\end{array}$ & $\begin{array}{r}15(6 \%) \\
236(94 \%)\end{array}$ & $<0.01$ \\
\hline $\begin{array}{l}\text { Pumpe } \\
\text { Zentrifugal } \\
\text { Roller } \\
\text { Nein } \\
\end{array}$ & $\begin{array}{r}2784(84 \%) \\
491(71 \%) \\
48(1 \%) \\
\end{array}$ & $\begin{array}{r}215(86 \%) \\
31(12 \%) \\
6(2 \%) \\
\end{array}$ & 0.45 \\
\hline $\begin{array}{l}\text { Kardioplegie } \\
\text { Bretschneider } \\
\text { Blutkardioplegie } \\
\text { Nein } \\
\end{array}$ & $\begin{array}{r}1462(44 \%) \\
1758(53 \%) \\
103(3 \%) \\
\end{array}$ & $\begin{array}{r}116(46 \%) \\
129(51 \%) \\
6(2 \%) \\
\end{array}$ & 0.74 \\
\hline $\begin{array}{l}\text { Temperatur } \\
\text { Normothermie } \\
\text { Milde Hypothermie } \\
\text { Tiefe Hypothermie }\end{array}$ & $\begin{array}{r}2554(76 \%) \\
734(22 \%) \\
65(2 \%) \\
\end{array}$ & $\begin{array}{r}177(71 \%) \\
63(25 \%) \\
11(4 \%) \\
\end{array}$ & $<0.01$ \\
\hline $\begin{array}{l}\text { Operationen } \\
\text { Andere } \\
\text { Nur Bypass } \\
\text { Nur Herzklappen } \\
\text { Beides }\end{array}$ & $\begin{array}{r}245(7 \%) \\
2037(61 \%) \\
596(18 \%) \\
445(13 \%) \\
\end{array}$ & $\begin{array}{r}18(7 \%) \\
153(61 \%) \\
30(12 \%) \\
50(20 \%) \\
\end{array}$ & $<0.01$ \\
\hline
\end{tabular}

Tab.11 kategorielle Parameter und ANV 
Eine Bypassoperation ist signifikant mit dem Auftreten eines ANV azzoziiert $(p=0,02)$. Die Klappenoperationen haben in dieser Analyse allerdings keine signifikante Beziehung mit dem Auftreten eines ANV ( $p=0,1)$, im Gegensatz zum ersten Teil der Analyse bezüglich eines Nierenersatzverfahrens, wo eine signifikante Beziehung besteht. Auch die Geschlechterverteilung ist mit dem Auftreten eines ANV mit $p=0.62$ nicht signifikant korreliert.

Die Reoperationen allerdings waren mit 16\% der Fälle im Vergleich zu 7\% der Erstoperationen hochsignifikant mit dem Auftreten eines ANV vergesellschaftet. Ebenso stellen sich die Zahlen bei dem Einsatz einer IABP dar. 16\% der Patienten, die eine solche Kreislaufunterstützung benötigten, bekamen ein ANV. Im Gegensatz dazu waren es von den Patienten die keine IABP bekamen, nur 7\%. Abb.22 veranschaulicht den Zusammenhang, indem die Gruppe der Patienten, welche eine IABP erhielten und nicht erhielten, jeweils als $100 \%$ aufgetragen wurden. Somit wird besonders deutlich sichtbar, welchen unterschiedlichen Anteil die Patienten, die ein ANV entwickelten, in den beiden Gruppen ausmachen.

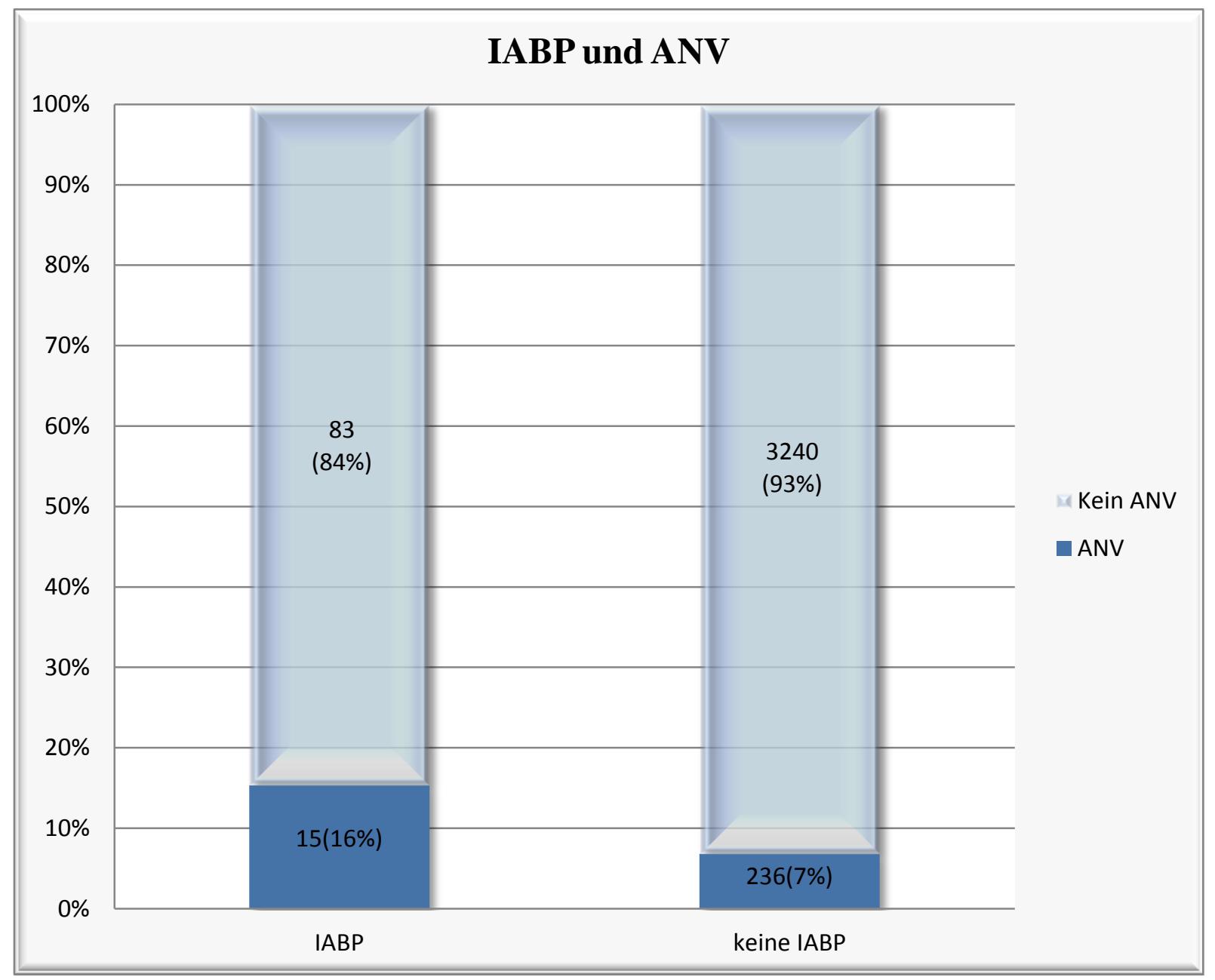




\section{Abb.22 IABP und ANV}

Die Herz-Lungen-Maschine wird in unserer Klinik z.T. mit Rollerpumpen (pulsatiler Fluss) und mit Zentrifugalpumpen (kontinuierlicher Fluss) angetrieben. Ein signifikanter Einfluss auf die Entwicklung eines ANV ließ sich zwischen diesen beiden Gruppen nicht nachweisen. Ebenso hatte die Art der Kardioplegie hinsichtlich des ANV keinen signifikanten Einfluss. Bei der Differenzierung zwischen den unterschiedlichen Operationen zeigte sich im Gegensatz zur Untersuchung bezüglich des NEV ein mit 7\% häufigeres ANV bei isolierten Bypassoperationen als mit 5\% bei isolierten Klappenoperationen. An erster Stelle standen wieder die Kombinationseingriffe, bei denen 10\% ein ANV entwickelten. Darauf folgten mit jeweils 7\% die Anderen Operationen und die Bypassoperationen. In der Abb.23 werden die Zusammenhänge zwischen den Gruppen deutlich.

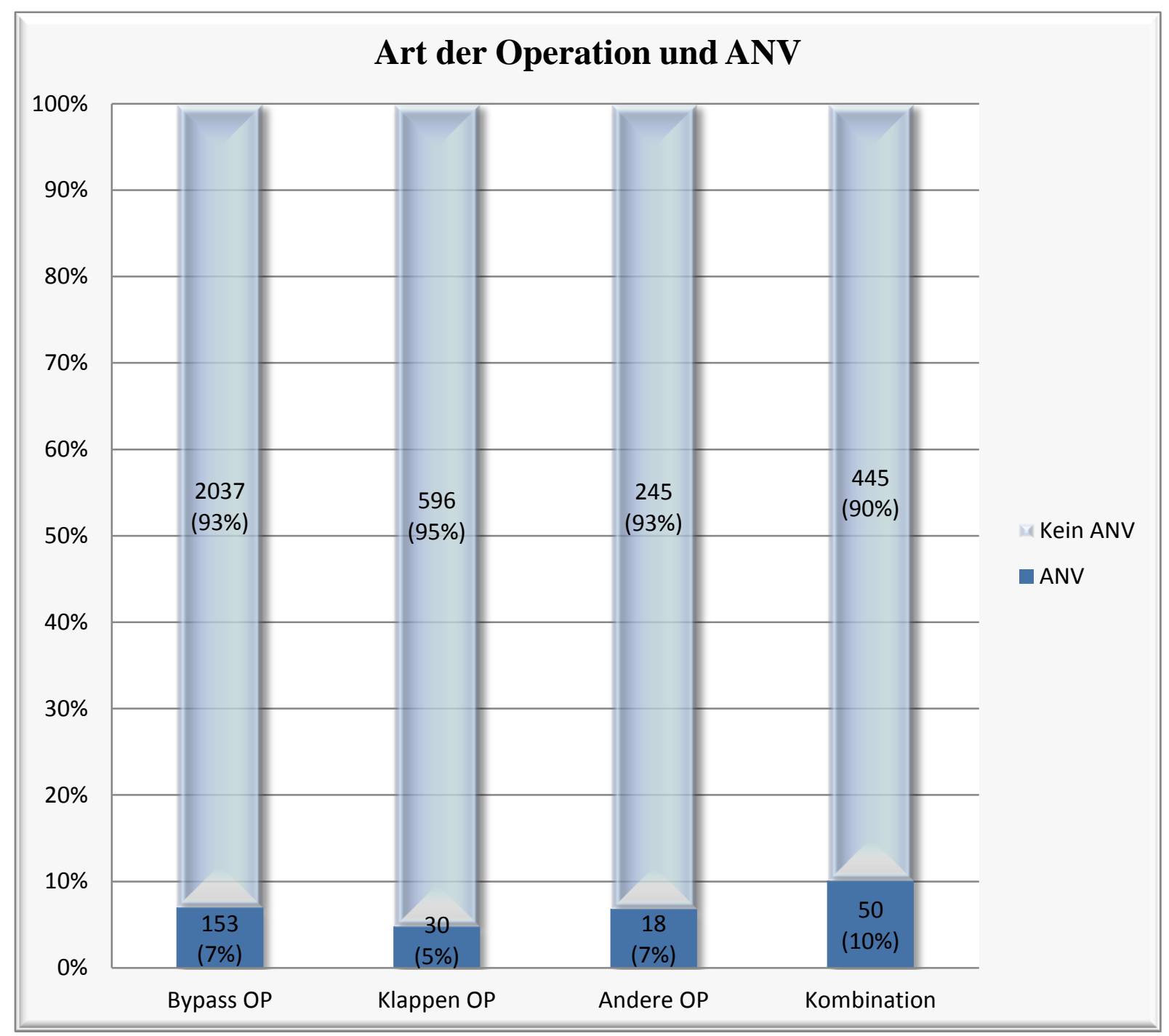

Abb.23 Art der Operation und ANV 
Bei der Analyse des Einflusses der Temperatur während der Herzoperation zeigten sich ebenfalls ähnliche Ergebnisse wie schon bei der Untersuchung bezüglich des NEV. Je niedriger die Temperatur desto höher war der Anteil an Patienten die ein ANV entwickelten. Die Zusammenhänge waren hochsignifikant. Abb. 24 zeigt, dass bei einer tiefen Hypothermie $\left(27-18^{\circ} \mathrm{C}\right)$ in $14 \%$ der Fälle ein ANV beobachtet wurde, gefolgt von $8 \%$ bei einer milden Hypothermie $\left(33-28^{\circ} \mathrm{C}\right)$ und $6 \%$ bei einer Normothermie $\left(37-34^{\circ} \mathrm{C}\right) . \mathrm{Ob}$ die Hypothermie selbst, oder die Prozedur die zur Abkühlung führt, diesen Effekt verursachen, bleibt zu klären.

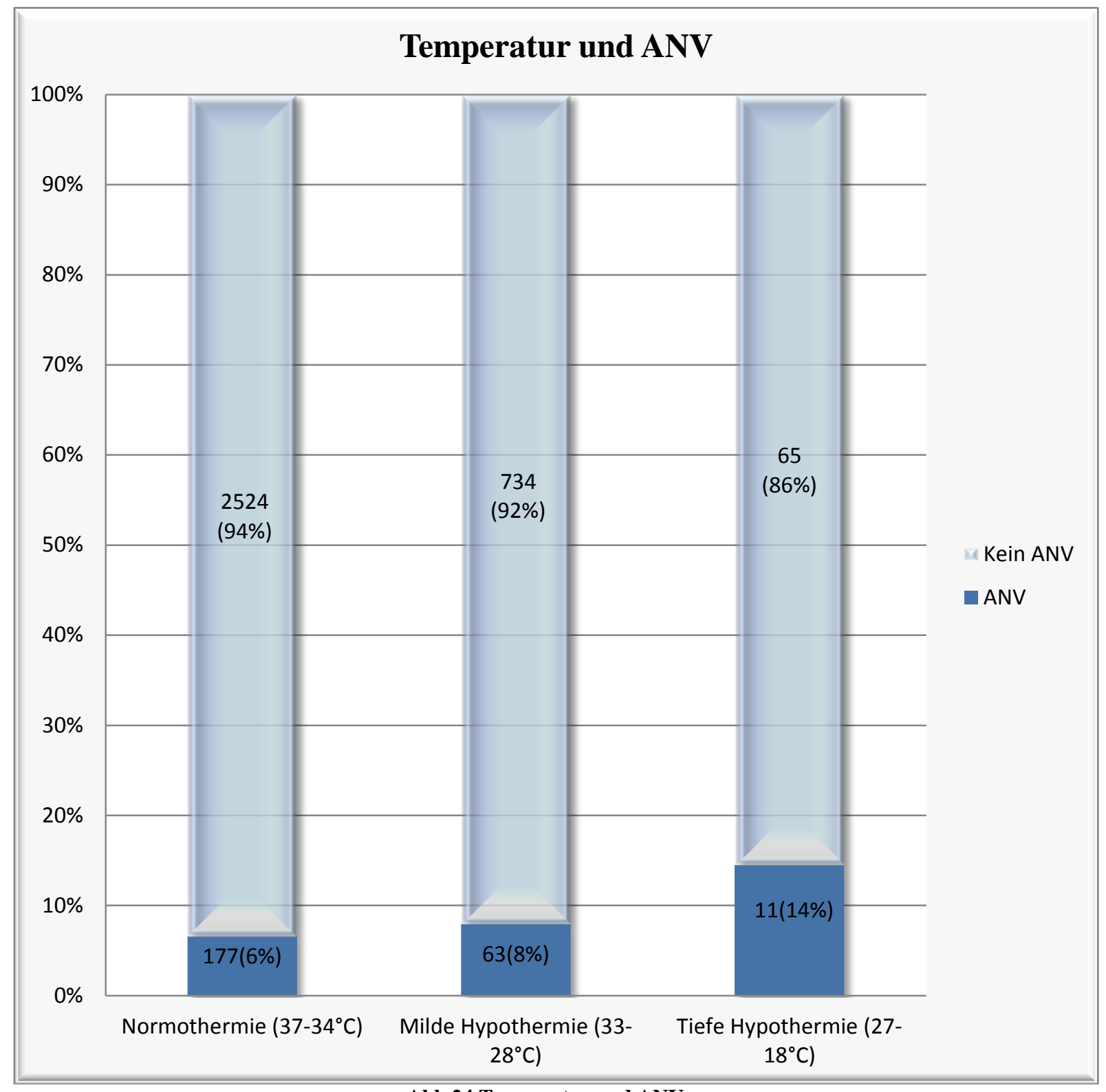

Abb.24 Temperatur und ANV 


\subsection{Einfluss der metrischen Parameter auf das Auftreten eines ANV gemäß RIFLE}

Die Ergebnisse für die metrischen Parameter sind in Tab.12 wiedergegeben. Sie zeigen bis auf die minimale Temperatur durchweg hoch signifikante Zusammenhänge mit dem Auftreten eines ANV. Sowohl das Alter als auch die Operationsdauer, Ischämiezeit, Perfusionszeit, Reperfusionszeit und die gesamten Blutverluste zeigen jeweils für die 25\%-, 50\%- und 75\%Quantile unterschiedliche Werte. Einige der Parameter werden im Folgenden zur graphischen Veranschaulichung zusätzlich in Box-Plots dargestellt.

\begin{tabular}{|c|c|c|c|}
\hline \multirow{2}{*}{ Parameter } & $(25 \%-, 50 \%-$ und 75\%-Quantil) & \multirow{2}{*}{ (Wilcoxon) } \\
\cline { 2 - 4 } & $\begin{array}{c}\text { Gruppe 1 } \\
(\mathrm{n}=3323)\end{array}$ & $\begin{array}{c}\text { Gruppe 2 } \\
(\mathrm{n}=251)\end{array}$ & p \\
\hline Alter (Jahre) & $61.0,68.0,74.0$ & $65.0,71.0,76.5$ & $<0.01$ \\
\hline Operationsdauer (min) & $229.0,274.0,329.0$ & $251.0,303.0,366.0$ & $<0.01$ \\
\hline Ischämiezeit (min) & $60.0,79.0,103.0$ & $64.0,85.0,114.5$ & $<0.01$ \\
\hline Perfusionszeit (min) & $98.0,126.0,160.0$ & $110.5,138.0,185.0$ & $<0.01$ \\
\hline Reperfusionszeit (min) & $28.0,37.0,49.0$ & $33.0,43.0,59.0$ & $<0.01$ \\
\hline Min. Temperatur $\left({ }^{\circ} \mathrm{C}\right)$ & $33.1,34.3,35.9$ & $32.7,34.0,35.9$ & 0.19 \\
\hline Blutverluste gesamt $(\mathrm{ml})$ & $470.0,800.0,1380.0$ & $910.0,1540.0,2830.0$ & $<0.01$ \\
\hline
\end{tabular}

Tab.12 metrische Parameter und ANV

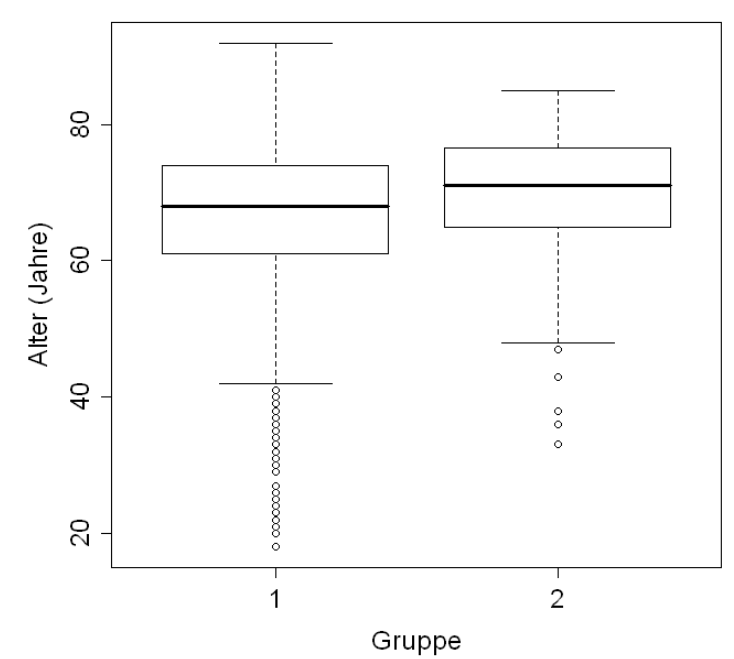

Abb.25 Alter und ANV
Der Box-Plot (Abb.25) für das Alter zeigt, dass die Patienten, die ein ANV entwickelten, älter sind (Median bei 71 Jahren) als diejenigen, die kein ANV bekommen (Median bei 68 Jahren). Die Beschriftung der Abbildung unterscheidet zwischen einer Gruppe 2, welche unserer Gruppe II mit einem ANV gemäß den RIFLEKriterien entspricht und einer Gruppe 1, welche alle anderen Patienten ohne ANV repräsentiert.

Bei der Ischämiezeit liegt der Median für die Patienten, die kein ANV bekamen bei 79 min im Gegensatz zu 85 min bei der Gruppe mit ANV. Bei der Perfusionszeit liegen die Mediane bei 126 min bzw. 138 min (siehe Abb. 26 und 27) 


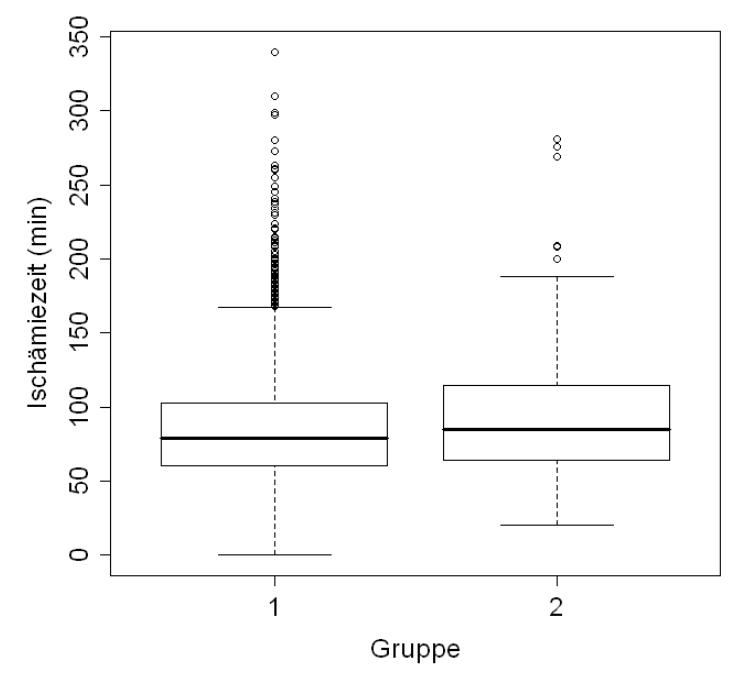

Abb.26 Ischämiezeit und ANV

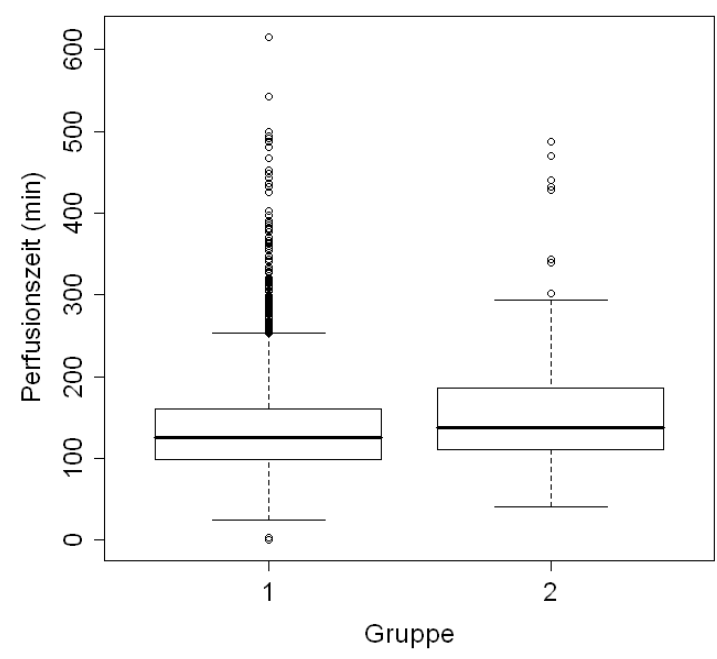

Abb.27 Perfusionszeit und ANV

Bei der Reperfusionszeit zeigt sich ein ähnliches Bild mit einem Median von 37 min bei der Gruppe 1 (kein ANV) im Vergleich zu einem Median von 44 min bei der Gruppe 2 (ANV). Die niedrigste Temperatur, die während der Operation erreicht wurde, ist ebenfalls mit dem Auftreten eines ANV assoziiert, wenn auch nicht in hochsignifikanter Weise $(p=0,19)$. Bei der Gruppe ohne ANV liegen die $25 \%$-, $50 \%$ - und $75 \%$ - Quantile bei $33,1^{\circ} \mathrm{C}, 34,3^{\circ} \mathrm{C}$, und $35,9^{\circ} \mathrm{C}$ in Abgrenzung an die entsprechenden Werte der Gruppe mit ANV, welche $32,7^{\circ} \mathrm{C}$, $34,0^{\circ} \mathrm{C}$ und $35,9^{\circ} \mathrm{C}$ betragen. Für die Gruppe mit ANV sind die Temperaturen also immer niedriger. (siehe Abb.29)

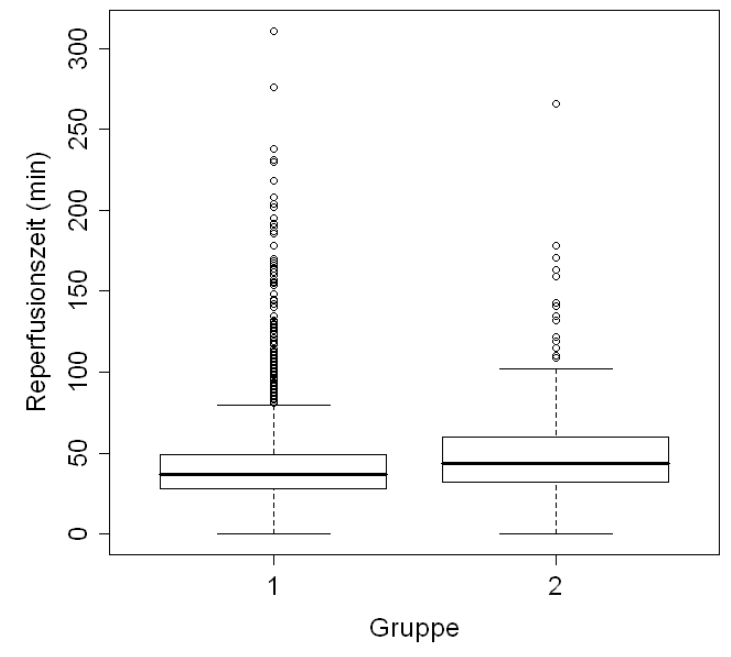

Abb.28 Reperfusionszeit und ANV

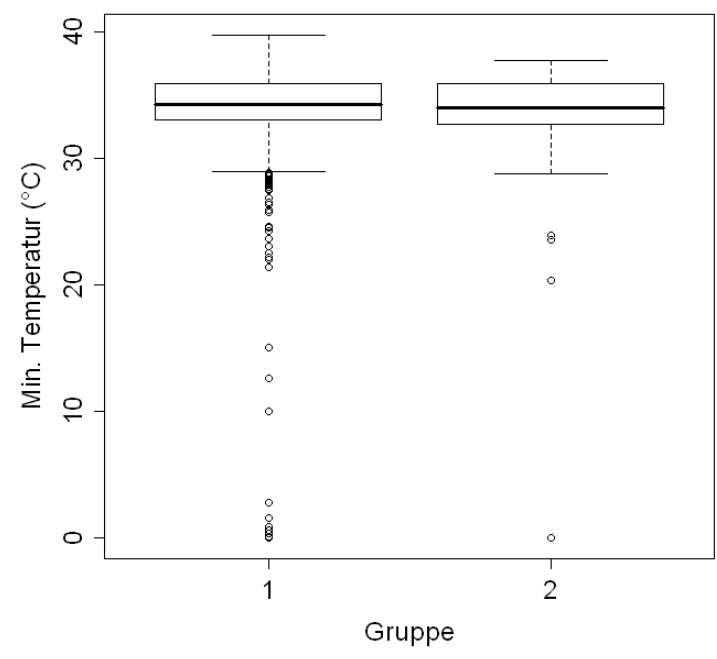

Abb.29 Minimale Temperatur und ANV 
Die Gesamtblutverluste sind in Anlehnung an die Ergebnisse der longitudinalen Analyse hier ebenfalls hochsignifikant mit einem ANV assoziiert. Der Median bei der Gruppe ohne ANV liegt bei $800 \mathrm{ml}$ und bei der Gruppe mit ANV deutlich höher bei 1540 ml. (siehe Abb. 30)

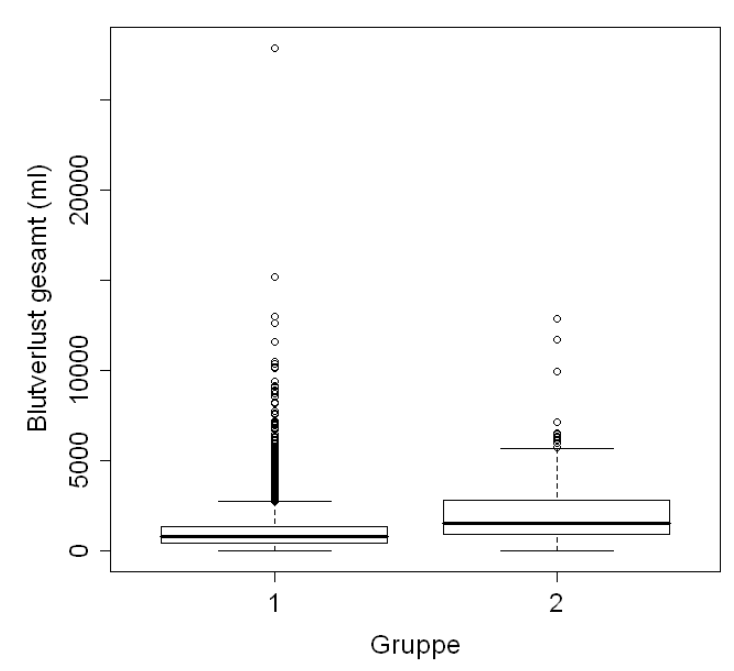

Abb. 30 Blutverluste und ANV

\subsection{Einfluss der kategoriellen Parameter auf den absoluten Kreatitininanstieg}

In den ersten beiden Teilen der Auswertung unserer Daten wurden alle Parameter auf die Zusammenhänge des Einsatzes eines NEV und dem Auftreten eines ANV untersucht. Die Indikation zum NEV, welche auch nach klinischen Gesichtspunkten gestellt wird, und die Einteilung in ein Nierenversagen gemäß der RIFLE-Kriterien haben natürlich den Nachteil, dass sie Patienten nur starr einer Gruppe zuordnen können oder nicht. Grenzfälle und latente Zusammenhänge werden dabei eventuell nicht in der Analyse berücksichtigt. Daher wurden für die wissenschaftliche Analyse zusätzlich nochmals alle Parameter mit dem absoluten Kreatininanstieg in Verbindung gesetzt, um auch latente Zusammenhänge zu beleuchten. Die Ergebnisse bestätigen im Wesentlichen die vorherigen Aussagen und verdeutlichen auch die Korrelation zwischen einem Kreatininanstieg und dem Abfall der Nierenfunktion bis hin zur Definition eines ANV nach RIFLE oder der Indikation eines NEV. Genauere Ergebnisse lieferte jedoch die Untersuchung des Pumpentyps und der Kardioplegielösung. Bezogen auf den absoluten Anstieg des Serumkreatinins gibt es signifikante Unterschiede zwischen der Zentrifugalpumpe, welche im 75\%- Quantil einen Anstieg von 0,5 mg/dl zeigt und der Rollerpumpe mit einem dementsprechenden Wert von 0,4 mg/dl. Der p-Wert für diese Berechnung beträgt 0,02 und ist damit signifikant. Bei dem Vergleich der 
Kardioplegielösungen zeigte die Lösung nach Bretschneider einen geringeren

Kreatininanstieg mit einem 75\%- Quantil von 0,4 mg/dl im Gegensatz zu 0,5 mg/dl bei der

Blutkardioplegie. In Tab.13 sind die einzelnen Wert aufgeführt.

\begin{tabular}{|c|c|c|c|}
\hline Parameter & $\begin{array}{c}\mathrm{n} \\
(\text { Anzahl) }\end{array}$ & $\begin{array}{l}\text { Kreatininanstieg (mg/ml) } \\
25 \%-, 50 \% \text { - und } 75 \% \text {-Quantil }\end{array}$ & $\begin{array}{c}\mathrm{p} \\
\text { (Wilcoxon, } \\
\text { Kruskal) }\end{array}$ \\
\hline $\begin{array}{l}\text { Geschlecht } \\
\text { M } \\
\text { W }\end{array}$ & $\begin{array}{l}2522 \\
1051\end{array}$ & $\begin{array}{l}0.0,0.1,0.4 \\
0.0,0.2,0.5\end{array}$ & $<0.01$ \\
\hline $\begin{array}{l}\text { Bypass } \\
\text { Ja } \\
\text { Nein }\end{array}$ & $\begin{array}{c}2727 \\
846\end{array}$ & $\begin{array}{l}0.0,0.1,0.5 \\
0.0,0.1,0.4\end{array}$ & $<0.01$ \\
\hline $\begin{array}{l}\text { Klappen } \\
\text { Ja } \\
\text { Nein }\end{array}$ & $\begin{array}{l}1250 \\
2323 \\
\end{array}$ & $\begin{array}{l}0.0,0.2,0.6 \\
0.0,0.1,0.4\end{array}$ & $<0.01$ \\
\hline $\begin{array}{l}\text { Re-Op } \\
\text { Nein } \\
\text { Ja }\end{array}$ & $\begin{array}{l}3370 \\
203\end{array}$ & $\begin{array}{l}0.0,0.1,0.4 \\
0.1,0.5,1.4\end{array}$ & $<0.01$ \\
\hline $\begin{array}{l}\text { IABP } \\
\text { Nein } \\
\text { Ja }\end{array}$ & $\begin{array}{c}3475 \\
98\end{array}$ & $\begin{array}{l}0.0,0.1,0.4 \\
0.3,0.7,1.3\end{array}$ & $<0.01$ \\
\hline $\begin{array}{l}\text { Pumpe } \\
\text { Zentrifugal } \\
\text { Roller }\end{array}$ & $\begin{array}{c}2998 \\
522\end{array}$ & $\begin{array}{l}0.0,0.1,0.5 \\
0.0,0.1,0.4\end{array}$ & 0.02 \\
\hline $\begin{array}{l}\text { Kardioplegie } \\
\text { Bretschneider } \\
\text { Blutkardioplegie }\end{array}$ & $\begin{array}{l}1578 \\
1886\end{array}$ & $\begin{array}{l}0.0,0.1,0.4 \\
0.0,0.1,0.5\end{array}$ & 0.01 \\
\hline
\end{tabular}

\subsection{Einfluss der metrischen Parameter auf den absoluten Kreatininanstieg}

Bei den metrischen Parametern bestätigen sich ebenfalls die in den vorherigen Kapiteln dargestellten Zusammenhänge. Alter, Operationsdauer, Ischämiezeit, Perfusionszeit, Reperfusionszeit, minimale Temperatur und der Gesamtblutverlust sind alle signifikant mit einem höheren Kreatininanstieg nach Berechnung mit dem Pearson’schen Korrelationskoeffizient und p-Werten unter 0,01 assoziiert. In Tab.14 sind die Ergebnisse zusammengefasst. In den anschließend folgenden Diagrammen (Abb. 31, 32 und 33) sind diese Effekte graphisch am Beispiel der Ischämiezeit, der minimalen Temperatur und des Gesamtblutverlustes dargestellt. 


\begin{tabular}{|l|c|c|}
\hline \multicolumn{1}{|c|}{ Parameter } & $\begin{array}{c}\text { Korrelationskoeffizient } \rho \\
\text { (Pearson) }\end{array}$ & $\mathrm{p}$ \\
\hline Alter (Jahre) & 0.17 & $<0.01$ \\
\hline Operationsdauer (min) & 0.20 & $<0.01$ \\
\hline Ischämiezeit (min) & 0.18 & $<0.01$ \\
\hline Perfusionszeit (min) & 0.22 & $<0.01$ \\
\hline Reperfusionszeit (min) & 0.19 & $<0.01$ \\
\hline Min. Temperatur $\left({ }^{\circ} \mathrm{C}\right)$ & -0.07 & $<0.01$ \\
\hline Blutverlust gesamt $(\mathrm{ml})$ & 0.34 & $<0.01$ \\
\hline
\end{tabular}

Tab.14 Kreatinin, metrische Parameter

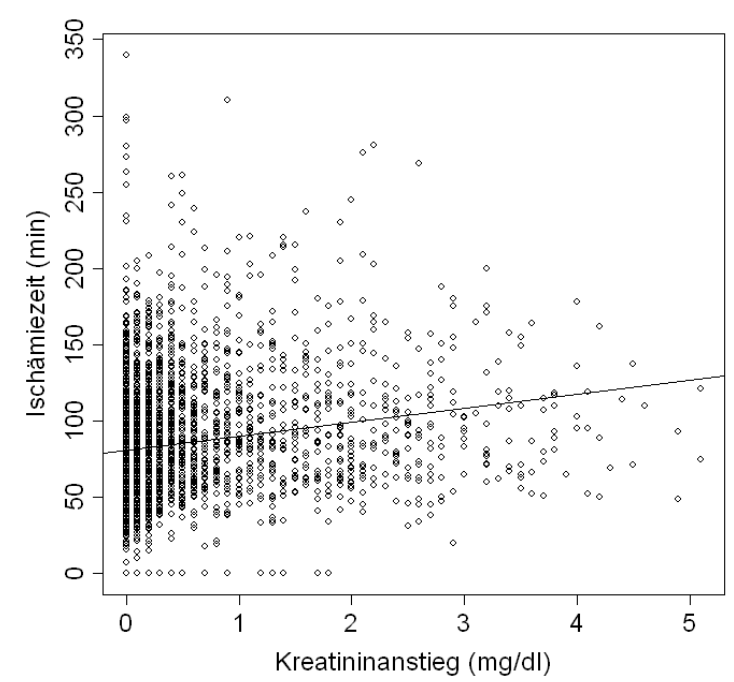

Abb.31 Ischämiezeit und Kreatinin

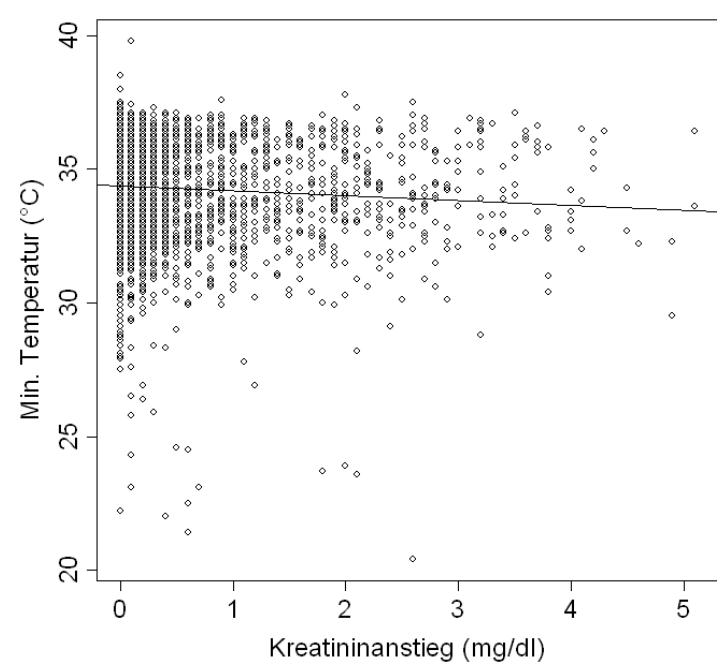

Abb.32 Min.Temperatur und Kreatinin

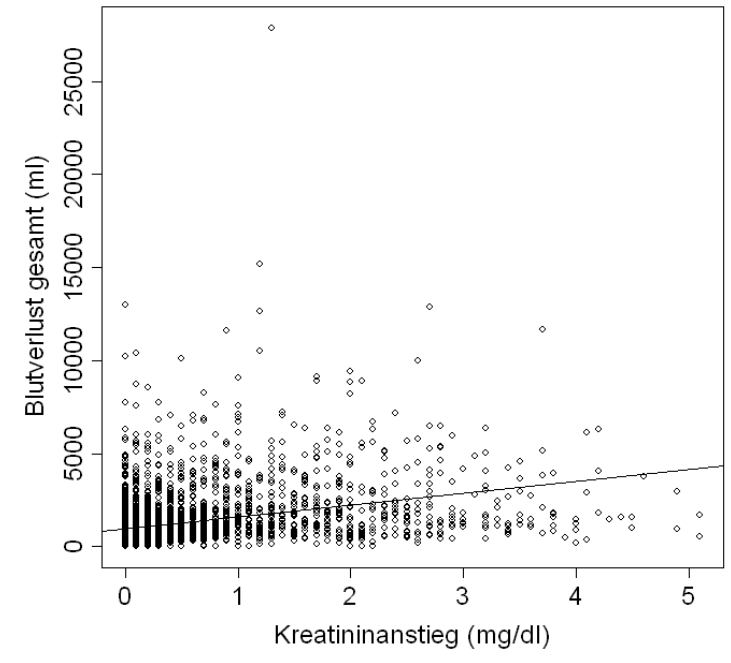

Abb.33 Blutverlust und Kreatinin 


\subsection{Zusammenfassung der Ergebnisse}

In Tabelle 15 sind alle Ergebnisse nochmals zusammengefasst. Dabei sind Zusammenhänge, bei denen eine signifikante Assoziation vorlag und deren $\mathrm{p}$-Werte dementsprechend unter 0,05 lagen, rot hervorgehoben. Die Parameter, bei denen alle drei durchgeführten Analysen hoch signifikante Zusammenhänge zeigten, waren: Re-Operation, IABP, Alter,

Operationsdauer, Ischämiezeit, Perfusionszeit, Reperfusionszeit und die Höhe der Blutverluste. Der Grad der Hypothermie und die Art der Operation zeigte sowohl bezüglich des NEV als auch des ANV nach RIFLE hoch signifikante Korrelationen.

\begin{tabular}{|c|c|c|c|}
\hline Parameter & $\begin{array}{c}\text { Gruppe 1 } \\
\text { (Nierenersatzverfahren) }\end{array}$ & $\begin{array}{c}\text { Gruppe } 2 \\
\text { (Def.RIFLE) }\end{array}$ & $\begin{array}{l}\text { Kreatininanstieg } \\
\text { (absolut) }\end{array}$ \\
\hline $\begin{array}{c}\text { Geschlecht } \\
\text { (W versus M) }\end{array}$ & $<0,01$ & 0,62 & $<0,01$ \\
\hline $\begin{array}{c}\text { Bypass } \\
(\mathrm{J} \text { versus N)} \\
\end{array}$ & 0,80 & 0,02 & $<0,01$ \\
\hline $\begin{array}{l}\text { Klappen } \\
(\mathrm{J} \text { versus N) }\end{array}$ & $<0,01$ & 0,10 & $<0,01$ \\
\hline $\begin{array}{c}\text { Re-OP } \\
(\mathrm{J} \text { versus N) }\end{array}$ & $<0,01$ & $<0,01$ & $<0,01$ \\
\hline $\begin{array}{c}\text { IABP } \\
(\mathrm{J} \text { versus } \mathrm{N})\end{array}$ & $<0,01$ & $<0,01$ & $<0,01$ \\
\hline $\begin{array}{c}\text { Pumpe } \\
\text { (Zentrifugal versus Roller) }\end{array}$ & 0,89 & 0,45 & 0,02 \\
\hline $\begin{array}{c}\text { Kardioplegie } \\
\text { (Blut versus Bretschneider) }\end{array}$ & 0,20 & 0,74 & 0,01 \\
\hline $\begin{array}{c}\text { Temperatur } \\
\text { (Grade der Hypothermie) }\end{array}$ & $<0,01$ & $<0,01$ & \\
\hline $\begin{array}{c}\text { Operationen } \\
\text { (Kombi,Andere,Klappen,Bypass) }\end{array}$ & $<0,01$ & $<0,01$ & \\
\hline $\begin{array}{c}\text { Alter } \\
(\text { Jahre })\end{array}$ & $<0,01$ & $<0,01$ & $<0,01$ \\
\hline $\begin{array}{c}\text { Kreatinin A } \\
(\mathrm{mg} / \mathrm{dl})\end{array}$ & $<0,01$ & & \\
\hline $\begin{array}{c}\text { Kreatinin B } \\
(\mathrm{mg} / \mathrm{dl})\end{array}$ & $<0,01$ & & \\
\hline $\begin{array}{c}\text { Operationsdauer } \\
(\mathrm{min})\end{array}$ & $<0,01$ & $<0,01$ & $<0,01$ \\
\hline $\begin{array}{l}\text { Ischämiezeit } \\
(\mathrm{min})\end{array}$ & $<0,01$ & $<0,01$ & $<0,01$ \\
\hline $\begin{array}{c}\text { Perfusionszeit } \\
(\mathrm{min})\end{array}$ & $<0,01$ & $<0,01$ & $<0,01$ \\
\hline $\begin{array}{c}\text { Reperfusionszeit } \\
(\mathrm{min})\end{array}$ & $<0,01$ & $<0,01$ & $<0,01$ \\
\hline $\begin{array}{c}\text { Min.Temperatur } \\
\left({ }^{\circ} \mathrm{C}\right)\end{array}$ & $<0,01$ & 0,19 & $<0,01$ \\
\hline $\begin{array}{c}\begin{array}{c}\text { Blutverlust } \\
(\mathrm{ml})\end{array} \\
\end{array}$ & $<0,01$ & $<0,01$ & $<0,01$ \\
\hline Zeitverlauf & $<0,01$ & & \\
\hline Wechselwirkung & $<0,01$ & & \\
\hline
\end{tabular}

Tab.15 Zusammenfassung Ergebnisse 
Hierbei war eine niedrige Temperatur mit dem vermehrten Auftreten eines ANV assoziiert. Hinsichtlich der Art der Operation waren die Kombinationseingriffe vor den ,anderen Operationen“, gefolgt von den Klappenoperationen und letztlich den Bypassoperationen signifikant mit dem Auftreten eines ANV vergesellschaftet. Zwischen der Zentrifugalpumpe und der Rollerpumpe gab es nur im Hinblick auf den absoluten Kreatininanstieg einen signifikanten Unterschied. Die Zentrifugalpumpe führte dabei zu einem stärkeren Anstieg der Kreatininwerte $(\mathrm{p}=0,02)$ als die Rollerpumpe. Bei der Wahl der Kardioplegielösung führte die Blutkardioplegie zu einem signifikant höheren Anstieg des Serumkreatinins als die Kardioplegie nach Bretschneider ( $\mathrm{p}=0,01)$. Bezüglich des NEV und ANV ergaben die Pumpe und Kardioplegielösung keine signifikanten Unterschiede. Die Blutverluste waren nicht nur in der absoluten Höhe hoch signifikant mit dem Auftreten eines akuten Nierenversagens vergesellschaftet. In der longitudinalen Untersuchung der Blutverluste über 6 Tage postoperativ war auch der Zeitverlauf und die Wechselwirkung zwischen den Zeitverläufen signifikant verschieden. 


\section{Diskussion}

\subsection{Allgemeine Aspekte}

\subsubsection{Gesichtspunkte zur Inzidenz / Mortalität / Definition des ANV}

Das Bestreben, die Ursachen und Zusammenhänge bei der Entstehung eines ANV im Zuge kardiochirurgischer Operationen besser zu verstehen, ist nach wie vor sehr groß. Das Auftreten eines ANV ist mit hohen Kosten (Harrison et al. 2006), weitreichenden Komplikationen und Organdysfunktionen assoziiert, die trotz verbesserter Behandlungsmöglichkeiten eine sehr hohe Mortalität aufweisen. Obwohl die Indidenz trotz einer verbesserten Risikoeinschätzung und Evaluierung der Patienten präoperativ in den letzten Jahren gesunken ist, blieb die Mortalität der Patienten, die ein ANV entwickelten, hoch (Landonie et al. 2006). Die in der Literatur angegebenen statistischen Maßzahlen sind aber wie schon erwähnt, einigen Ungenauigkeiten unterworfen. Die in der Literatur beschriebene Bandbreite von Inzidenzen des ANV nach kardiochirurgischen Operationen von 0,7-31\% (Thakar et al. 2003b) und Mortalitätsraten von über 50-60\% (Garwood et al. 2004) oder bis zu 90\% (Landonie et al. 2006) kann durch die unterschiedlichen Voraussetzungen und verschiedenen Definitionen eines ANV in den jeweiligen Studien teilweise erklärt werden.

In einigen Studien wird das ANV nur über den Bedarf eines Nierenersatzverfahrens (NEV) definiert. Rastan et al. (2006) untersuchten die Effekte von Notfalleingriffen am nicht schlagenden bzw. schlagenden Herz hinsichtlich der postoperativen Nierenfunktion, welche dem Bedarf eines NEV gleichgesetzt wurde. Bei Bahar et al. (2005) wird die Inzidenz des ANV als Anzahl der postoperativ benötigten NEV mit 1,16\% (168 Patienten) bei einer Gesamtanzahl von 14437 Patienten angegeben. Die Mortalität in dieser Gruppe betrug 79,7\%. In einer der umfangreichsten Studien von Chertow et al. (1998) mit 42773 Patienten wurde das ANV ebenfalls als Bedarf eines NEV, in diesem Fall innerhalb der ersten 30 Tage postoperativ, definiert. Die dabei errechnete Inzidenz betrug 1,1\% mit einer Mortalität von 63,7\%. Auch bei Frost et al. (1992) wurde das ANV als Bedarf an NEV definiert und eine dementsprechende Inzidenz von 3,4\% bei einem Stichprobenumfang von 1988 errechnet. Die Mortalität in dieser Gruppe betrug 63\%.

In einer Reihe weiterer Studien wurde neben der Notwendigkeit eines NEV postoperativ eine erweiterte Definition des ANV angewandt, die auch Patienten mit bestimmten Veränderungen 
der Serumkreatininwerte ohne Bedarf eines NEV in die Gruppe des ANV mit einbezog. Beispiele hierfür sind Andersson et al. (1993), welche neben dem NEV ein ANV als Anstieg des Serumkreatinins von über $50 \%$ vom präoperativen auf den postoperativen Wert definierten. Dementsprechend wurden Mortalitätsraten für einen Serumkreatininanstieg unter $50 \%$ von $0,4 \%$, einem Anstieg über 50\% von 1,3\% und bei NEV von $44 \%$ errechnet. Auch Mangos et al. (1995) definierten das ANV anhand des Serumkreatinins auf folgende Weise: Bei Patienten, welche präoperativ einen Kreatininwert von kleiner gleich 0,78mg/dl hatten, wurde ein postoperatives ANV als Verdopplung des Ausgangswertes definiert, vorausgesetzt der Wert war nach wie vor über $0,78 \mathrm{mg} / \mathrm{dl}$. Bei allen anderen Patienten wurde ein postoperatives ANV als Kreatininanstieg größer gleich 0,6mg/dl definiert. Für die erste Gruppe wurde dabei eine Inzidenz von 1,1\% und für die zweite von 16\% errechnet. $20 \%$ der Patienten benötigten eine Dialysebehandlung. Die Gesamtmortalität nach dieser Definition des Nierenversagens war 13\%. Thakar et al. (2005) haben neben dem NEV für die Definition des ANV anstelle des Kreatininwertes einen Abfall der GFR, abgeschätzt aus der Kreatininclearance, von mehr als 50\% angewandt. Die Inzidenz für ein NEV war 1,82\% und die Gesamtmortalität lag bei 2,2\% im Vergleich zu 61,2\% der Patienten mit NEV. In der Studie von Conlon et al. (1999) wurde ebenfalls neben dem NEV eine zweite Gruppe mit ANV über einen Serumkreatininanstieg von $1 \mathrm{mg} / \mathrm{dl}$ vom Grundwert definiert. In der Gruppe mit NEV lag die Inzidenz bei 0,7\% und die Mortalität bei 28\%. In der Gruppe mit dem Kreatininanstieg ohne NEV war die Inzidenz 7,9\% und die Mortalität 14\%. Khiji et al. (2004) definierten ein ANV mit dem postoperativen Anstieg des Serumkreatinins von über 2,5mg/dl, welches in ihrer Studie eine Inzidenz von 7\% aufweist. Die Mortalität in dieser Gruppe wird mit $88,8 \%$ angegeben.

Anhand dieser Ausführungen wird deutlich, wie vielfältig die Definitionen für ein ANV in der Literatur sind und dass die Ergebnisse und Maßzahlen im Zuge dieser Erkenntnis vorsichtig zu bewerten sind. Mit dem Ziel, diese Unstimmigkeiten in der Definition des ANV zu vereinheitlichen und einen internationalen Konsensus zu schaffen, wurden im Jahr 2004 die RIFLE-Kriterien definiert, die eine umfassende und vergleichbare Einschätzung der Nierenfunktion erlauben (Venkataraman et al. 2007). Die Definition ruht auf zwei unabhängigen Säulen, nämlich dem Kriterium der glomerulären Filtration und dem Kriterium der Urinausscheidung (siehe auch 1.3.1.3).

In dieser Arbeit wurde daher entschieden, neben der Definition einer Gruppe über den Bedarf eines NEV im Zuge einer postoperativen Nierenfunktionseinschränkung auch eine Gruppe 
über die aktuelle Definition des ANV anhand der RIFLE-Kriterien zu bilden. Beide Gruppen wurden dann mit allen zu untersuchenden Parametern auf signifikante Zusammenhänge untersucht. Zusätzlich wurden noch alle Variablen mit dem absoluten postoperativen Serumkreatininanstieg verglichen und auch hier signifikante Unterschiede zwischen den Gruppen herausgearbeitet.

\subsubsection{Inzidenz / Mortalität unserer Studie im Kontext der Literatur}

Im Zuge dieser Untersuchungen ergaben sich in der vorliegenden Arbeit folgende statistische Maßzahlen. Von insgesamt 3574 operierten Patienten entwickelten 365 postoperativ ein Nierenversagen, welches der Behandlung mit einem NEV bedurfte. Das entspricht einer Inzidenz des ANV gemäß Indikation zum NEV von 10\%. Dabei ist zu beachten, dass die Indikation zur Hämofiltration über den Untersuchungszeitraum keineswegs einheitlich geregelt war. Die Indikation zum NEV wurde aufgrund der damaligen Datenlage in den ersten Jahren des Untersuchungszeitraums (2000-2005) wesentlich früher und aggressiver gestellt als dies heute der Fall ist. Die aktuelle Indikation zum NEV im Klinikum Göttingen ist unter Punkt 3.1 erläutert. Die Inzidenz des ANV anhand der Kriterien nach RIFLE beträgt in unserer Studie 32\%. Das heißt, von 3574 operierten Patienten entwickelten 1156 eines oder mehrere der Kriterien nach RIFLE für ein ANV (erhöhtes Serumkreatinin um Faktor 3 oder Abfall der GFR um 75\% oder Serumkreatininwert von $4 \mathrm{mg} / \mathrm{dl}$ ). Hierbei gilt es also zu beachten, dass die RIFLE-Kriterien neben dem Serumkreatinin auch die abgeschätzte GFR mit einbeziehen, wie von einigen Studien längst gefordert (Simon et al. 2007, Noyez et al. 2006, Wijeysundera et al. 2006). Die Gesamtmortalität in unserem Kollektiv betrug 6,4\% was 230 verstorbenen Patienten von insgesamt 3574 Operierten entspricht. Die Mortalität der Patienten mit einem ANV betrug mit 57 von 251 Patienten 22\%. Von den 365 Patienten, die eine NEV benötigten, verstarben 177, was einer Mortalität in dieser Gruppe von $48 \%$ entspricht. Die Zahlen für die Definition des ANV nach den RIFLE Kriterien ist schwer mit denen anderer Studien vergleichbar, da die Definitionen stark differieren und es keine vergleichbare Basis gibt. Für die Zahlen, die das ANV im Sinne eines NEV betrifft, liegen die Zahlen innerhalb der in der Literatur angegebenen Werte. Die Inzidenz von 10\% für ein NEV entspricht der in der Literatur genannten Bandbreite von 0,7\% bis 31\% (Thakar et al. 2003b). Doch aufgrund der unterschiedlichen Indikationen, die sich ja im Klinikum Göttingen sowie sicherlich auch in anderen Einrichtungen im Laufe des Untersuchungszeitraumes verändert haben, sollten auch diese Werte vorsichtig bewertet werden. Was die Mortalität des ANV mit NEV angeht, liegen die Zahlen in unserer Studie mit 48\% im Vergleich zu der in der Literatur eher niedrig. Landoni et al. (2006) geben eine Mortalität von bis zu 90\% an und Garwood et 
al. (2004) eine Mortalität, die in den meisten Fällen bei 50-60\% liegt, diese wohl aber auch übertrifft. Zusammenfassend lässt sich festhalten, dass mit unserer Untersuchung sowohl die Inzidenz wie auch die Mortalität, welche mit einem NEV in Zusammenhang gesehen wurde, vergleichbare Angaben in der Literatur bestätigt.

\subsubsection{ANV als multifaktorielles Geschehen}

Ein weiterer besonderer Gesichtspunkt bei der Untersuchung des ANV ist seine komplexe Pathophysiologie. Die Tatsache, dass Patienten, die sich einer Herzoperation unterziehen müssen, ein relativ hohes Risiko haben, ein ANV zu entwickeln, lässt sich nur zum Teil durch die Auswirkungen der extrakorporalen Zirkulation erklären. Die Entwicklung eines ANV ist ein multifaktorielles Geschehen, auf das mannigfaltige prä- und perioperative Faktoren Einfluss nehmen. In verschiedenen Studien sind signifikante Zusammenhänge zwischen dem Auftreten eines postoperativen ANV und den unterschiedlichsten Parametern festgestellt worden. Bahar et al. (2005) und Thakar et al. (2005) lieferten solche Ergebnisse unter anderem für das weibliche Geschlecht, Alter, Diabetes mellitus, Bluthochdruck, eingeschränkte Nierenfunktion bzw. erhöhte Serumkreatininwerte, Herzinsuffizienz bzw. verminderte Ejektionsfraktion des linken Ventrikels, Einsatz von IABP, Kombinationseingriffe, Klappenoperationen, Notoperationen, Reoperationen, lange Ischämiezeiten, lange Operationszeiten, Hypothermie, Re-Exporation bei Blutung, postoperativen systemischen oder mediastinalen Infektionen, Einsatz von inotropen Medikamenten, COPD (chronic obstructive pulmonary disease $=$ chronisch obstruktive Lungenerkrankung), ,ethnischer“ Zugehörigkeit, peripherer arterieller Verschlusskrankheit und Karotidenauskultationsgeräusch. Viele dieser Einflussfaktoren fanden mittlerweile Eingang in mehrfach an großen Kollektiven bestätigten Scores und auch in unserer Arbeit konnten einige dieser Parameter untersucht und bestätigt werden.

Es wird aber auch deutlich, dass durch die Vielfältigkeit der Einflussfaktoren und der komplexen Physiologie der Niere (siehe 2.2.2) bezüglich des ANV und seiner Entstehung (siehe 2.3.3) eine definitive und unabhängige Zuordnung einer Einflussgröße extrem schwierig ist. Um dies zu bewirken, müssten alle Parameter in ihrer Gesamtheit gekannt und so adjustiert und korrigiert werden, dass die Stichprobe exakt vergleichbar wird. Bei all unseren Ergebnissen ist es dementsprechend zwar möglich, nach den Regeln der statistischen Berechnung signifikante Zusammenhänge oder Unterschiede zwischen Variablen aufzuzeigen, aber die Interpretation sollte immer unter Berücksichtigung der Tatsache erfolgen, dass zusätzliche Faktoren eine verstärkende oder abschwächende Wirkung ausüben 
könnten. Allein das zunehmende Alter der operierten Patienten bringt eine Anhäufung von zusätzlichen Einflussgrößen im Sinne von Grundleiden und Vorerkrankungen bzw.

Voreingriffen mit sich, die mit hoher Wahrscheinlichkeit Auswirkungen auf die Entstehung eines ANV haben.

Diese Erkenntnis, gepaart mit der Hypothese, dass ein ANV medikamentös nicht behandelbar ist, da die Nierenfunktion durch Pharmazeutika nicht wieder hergestellt werden kann, verlangt eine besondere Beachtung präventiver Maßnahmen und Risikostratifizierungen bereits vor der Operation (Harrison et al. 2005). Die Darstellung der präventiven Maßnahmen ist unter dem Punkt 2.3.7.1 aufgeführt. Trotzdem soll hier nochmal die besondere Relevanz der Aufrechterhaltung einer normalen Nierenperfusion im Sinne einer Volumensubstitution und Gabe von Medikamenten zur Erhaltung einer stabilen hämodynamischen Situation erwähnt werden. Der Einsatz spezieller Medikamente zur Prävention eines ANV wie Dopamin, Mannit, Schleifendiuretika wie beispielsweise Furosemid, Kalziumantagonisten, Prostaglandine oder atriales natriuretisches Peptid (ANP) werden in der Literatur sehr kritisch beurteilt und letztlich nicht oder nur sehr vorsichtig empfohlen (Garwood et al. 2004). Die Gabe von Dopamin, auch niedrig dosiert, ist mittlerweile obsolet. Diverse Studien belegen, dass es keinen positiven Effekt auf die Entstehung eines ANV hat. (Bellomo et al. 1994, Garwood et al. 2004). Furosemid hat bei kurzlebigen oligurischen Nierenfunktionseinschränkungen ohne Indikation zur Dialyse noch eine gewisse Berechtigung zur Aufrechterhaltung der Diurese. Allerdings sollte es aufgrund von ebenfalls vorhandenen schädlichen Effekten auf die Nierenfunktion zurückhaltend eingesetzt werden (Garwood et al. 2004). ANP und BNP (= brain natriuretic peptide) sind noch in der Erprobung, könnten aber sinnvolle Alternativen darstellen. 


\subsection{Beziehung der Ergebnisse zum aktuellen Forschungsstand}

Bereits unter Punkt 2.4 der vorliegenden Arbeit wurde der aktuelle Stand der Forschung zum ANV nach kardiochirurgischen Operation dargestellt und im ersten Teil der Diskussion wurden allgemeine Aspekte im Lichte der Literatur erläutert. Deshalb soll an dieser Stelle insbesondere die Einfügung der Ergebnisse dieser Arbeit in den Kontext der bisherigen Forschungen erfolgen. Die anderen Parameter, die nicht in unserer Arbeit vorkommen aber eine Rolle spielen, wurden bereits in den vorherigen Kapiteln abgehandelt.

\subsubsection{Personen-und Operations-assoziierte Parameter}

In der vorliegenden Arbeit wurden Ergebnisse in Bezug auf das Auftreten eines ANV nach Herzoperationen bezüglich des Geschlechts, Alters, Art der Operation, Reoperation, Verwendung einer IABP und des Blutverlustes herausgearbeitet.

Das weibliche Geschlecht war in der vorliegenden Studie hochsignifikant mit dem Auftreten eines ANV sowohl im Sinne eines NEV als auch einem ANV nach Definition der RIFLEKriterien mit p-Werten von jeweils $<0,01$ assoziiert. Auch in Bezug auf den absoluten Kreatininanstieg gab es einen signifikanten Unterschied zwischen Männern und Frauen mit einem p-Wert < 0,01. Bei dem Einsatz von NEV waren es von 2522 operierten Männern 234, welche dieses benötigten, im Gegensatz zu 131 von 1051 operierten Frauen. In Prozent entspricht dies 12\% der Frauen im Vergleich zu 9\% der Männer. Bezüglich des ANV nach RIFLE ergab sich ein ähnlicher Zusammenhang: 30\% der Männer und 38\% der Frauen entwickelten ein ANV. Dieses Ergebnis ist in der Literatur ebenfalls beschrieben worden, beispielsweise von Thakar et al. (2005), welche das weibliche Geschlecht sogar als unabhängigen Risikofaktor für ein ANV und eine hohe Mortalität bei der Entwicklung eines Risikoscores für ein ANV werten. Bei Thakar et al. (2005) wurden 24660 am Herzen operierte Patienten untersucht und bei einer Definition des ANV als Dialysebedarf oder 50\% Abfall der GFR eine Inzidenz des ANV mit NEV von 1,82\% beschrieben. Dabei waren Frauen mit 2,36\% häufiger betroffen als Männer mit 1,60\%. Dies entsprach einem p-Wert von $<0,0001$. Die Mortalität der Patienten, welche ein NEV erhielten, lag bei 61,2\%, wobei Frauen einen Anteil von 68,6\% und Männer einen Anteil von 56,5\% hatten. Dies entspricht einem p-Wert von 0,01. Obwohl andere Studien (Bahar et al. 2005) keinen signifikanten Unterschied zwischen den Geschlechtern identifizieren können, sind unsere Ergebnisse 
diesbezüglich relativ eindeutig und auch der große Stichprobenumfang und die Ergebnisse von Thakar et al. (2005) machen diesen Zusammenhang sehr wahrscheinlich.

Im Vergleich hierzu gibt es in Bezug auf das Alter der Patienten einen eindeutigen Befund in der Literatur, welcher unsere Ergebnisse ebenfalls unterstreicht. In unsere Studie wurden nur Erwachsene ab 18 Jahren einbezogen, der jüngste operierte Patient in unserem Kollektiv war dementsprechend 18 Jahre alt. Der älteste operierte Patient war 92 Jahre alt, und der Median des Alters lag bei 68 Jahren. 73\% der Patienten waren zwischen 60 und 80 Jahre alt. Das Alter der Patienten mit NEV war signifikant höher als das derer, die kein NEV benötigten. Die 25\%-, 50\%- und 75\%- Quantile für das Alter der Patienten ohne NEV betrugen 61, 68 und 74 Jahre im Vergleich zu denen mit NEV von 66, 71 und 76 Jahren. Dieser Unterschied war hochsignifikant mit einem $\mathrm{p}$-Wert nach Wilcoxon von $<0,01$. Bezüglich der Definition des ANV nach RIFLE ergab sich Ähnliches. Der Median des Alters der Patienten ohne ANV war mit 66 Jahren deutlich unter dem der Patienten mit ANV von 71 Jahren. Auch hier war der Zusammenhang mit $\mathrm{p}<0,01$ hochsignifikant. In diversen Studien wurde dieser Zusammenhang bestätigt. Bahar et al. (2005) beschreiben dieses Ergebnis in ihrer 14437 Patienten umfassenden Studie ebenso wie Andersson et al. (1993), Conlon et al. (1999), Heikkinen et al. (1985), Khiji et al. (2004), Thakar et al. (2005) und weitere. Auch in unserer Studie waren $41 \%$ der Patienten über 70 Jahre alt und es liegt nahe, dass Inzidenzen des ANV und dessen Mortalitätsraten auch durch diese Tatsache stark beeinflusst wurden. Die Wahrscheinlichkeit ist hoch, dass ältere Patienten mit mehr Grundleiden, die v. a. auch die Blutgefäße und damit die Hämodynamik betreffen, behaftet sind und die Entstehung eines ANV mit begünstigen. Unabhängig davon kommt es im Alter zu einer Abnahme der Nierenperfusion, welche nicht nur durch eine veränderte Hämodynamik sondern auch durch eine Reduktion der Anzahl der Glomeruli verursacht sein könnte. Ginge man davon aus, dass bei älteren Patienten eine bloße Minimalfiltration ohne die beim jungen Menschen vorhandene Reservekapazität von ca. 200\% vorliegt, könnte dies erklären, warum deren Nierenfunktion unter dem Einfluss einer Herzoperation leichter kompromittierbar wäre. Ähnlich kongruente Zusammenhänge zwischen den Ergebnissen in der Literatur und unseren zeigten sich in Bezug auf eine Reoperation und den Einsatz einer intraaortalen Ballonpumpe zur Kreislaufunterstützung bei low cardiac output. In der vorliegenden Arbeit waren die Zusammenhänge dieser Parameter mit dem Auftreten eines ANV sowohl als NEV als auch nach RIFLE-Kriterien besonders deutlich. 9\% der Erstoperierten haben im Vergleich zu 29\% der Reoperierten ein ANV im Sinne eines NEV entwickelt, was einem errechneten p-Wert 
von deutlich unter 0,01 entspricht. Bei Einsatz der IABP zeigen sich ebenfalls klare Unterschiede: Nur 9\% der Patienten ohne IABP bekamen ein NEV im Vergleich zu $41 \%$ der Patienten mit IABP. In Bezug auf ANV nach den RIFLE-Kriterien sind die Zahlen noch eindeutiger: 67\% der Patienten mit IABP haben ein ANV entwickelt im Vergleich zu 31\%, die kein IABP benötigten. Dies unterstreicht die in dieser und weiteren Arbeiten oftmals zitierte Hypothese, dass die hämodynamische Ausgangslage für die Nierenperfusion und damit deren Funktion von herausragender Bedeutung ist. Bei einer Kreislaufinstabilität, die eine IABP nötig werden lässt, ist in der Folge ein ANV höchst wahrscheinlich. Diese Ergebnisse wurden von verschiedenen Studien bestätigt. Garwood et al. (2004), Bahar et al. (2005) und Chertow et al. (1998) mit ihrer Studie an 42773 Patienten zeigten, dass eine Reoperation mit einem erhöhten Risiko für ein ANV einhergeht. Ebenfalls stellten sie signifikante Zusammenhänge zwischen dem Bedarf an einer IABP als Reaktion auf ein low cardiac output und dem Auftreten eines ANV her. Die hämodynamische Stabilität wird im Kontext der Literatur als entscheidender Faktor für die Nierenfunktion bewertet (Garwood et al. 2004). Damit ist eine zureichende Hämodynamik mit an die Situation angepasstem HZV und peripherem Widerstand gemeint, welche den Perfusionsdruck bestimmt und in Zusammenhang mit der Vorlast schlussendlich die Perfusion der Niere sicherstellt. Vor dem Hintergrund der von Plasmafluss, Blutdruck, onkotischer Zusammensetzung des Blutes und Filtrationskoeffizient abhängigen glomulären Filtration (Brenner et al. 1972), welche ihrerseits vollkommen die Blutversorgung des Tubulussystems bestimmt (Lameire und Vanholder 1998), wird der Stellenwert der Hämodynamik deutlich. Insbesondere, wenn man sich klarmacht, dass der einzige Antrieb für die Filtration und konsekutive der Blutversorgung des Tubulussystems die Pumpkraft des Herzens ist.

Signifikante Unterschiede gab es auch bei der Art der Operation. So konnten in dieser Arbeit die Klappeneingriffe als Risikofaktor mit einem signifikant höheren Anteil an ANV und NEV als die Gesamtheit der restlichen Eingriffe identifiziert werden. Von den Patienten unserer Analyse, die postoperativ ein NEV bekamen, hatten 13\% eine Klappenoperation und 9\% keine Klappenoperation hinter sich. Das ergibt, bezogen auf den Stichprobenumfang, einen pWert von $<0,01$ und ist damit hochsignifikant. Auch die Gruppe II mit der Definition des ANV nach RIFLE hat mit 38\% deutlich mehr Patienten mit Klappenoperation als ohne (29\%). Auch hier errechnet sich ein $\mathrm{p}-$ Wert von $<0,01$. In der Literatur ist dieses Ergebnis vielfach beschrieben worden. In den Studien von Heikkinen et al. (1985), Mangos et al. (1995) und Grayson et al. (2003) beispielsweise wird dieser Zusammenhang klar dargestellt. Bei der 1988 Patienten umfassenden Studie von (Heikkinen et al. 1085) mit seiner Definition des ANV als 
Bedarf eines NEV wurden als wichtigste Risikofaktoren für ein ANV die Perfusionszeit, postoperative Infektionen und Klappenoperationen dargestellt. Ebenfalls wurde in der Literatur (Bahar et al. 2005, Mangos et al. 1995) das besondere Risiko von

Kombinationseingriffen, die eine Klappenoperation beinhalten, beschrieben, welches wir in unserer Studie bestätigen können. Kombinationseingriffe an Herzklappen und Koronararterien waren mit 50\% am häufigsten mit einem ANV (Gruppe II) assoziiert. Dem folgten "andere" Operationen, die nicht die Herzklappen oder eine Revaskularisierung betrafen, mit 32\% vor den reinen Klappenoperationen und Revaskularisierungsoperationen mit jeweils 29\%. Bezüglich eines NEV waren ebenfalls die Kombinationseingriffe mit $17 \%$ vor den "anderen" Operationen mit 15\%, den Kappenoperationen mit 10\% und den Bypassoperationen mit $8 \%$ am stärksten vertreten. Die Unterschiede zwischen den Gruppen waren mit $\mathrm{p}$-Werten von jeweils $<0,01$ signifikant und verdeutlichen die besondere Stellung der Klappenoperationen vor allem in Kombination mit weiteren Eingriffen am Herzen, die im Bezug auf ein ANV als klarer Risikofaktor gewertet werden sollten. Die Konsequenz sollte sein, dass Patienten die solchen Operationen zugeführt werden, mit besonderen präventiven Maßnahmen und einer besonderen Überwachung der hämodynamischen Situation vor dem Auftreten eines ANV geschützt werden. Alle bis hierhin aufgeführten Parameter zeigten neben den signifikanten Unterschieden bezüglich eines NEV oder ANV (GruppeII) auch signifikante Unterschiede im absoluten Anstieg der Serumkreatininwerte. Von dem Graubereich einer latenten Nierenfunktionsstörung, in dem Kreatininwerte zu einer Überschätzung der Nierenfunktion führen können und die Anwendung der Kreatininclearance zu bevorzugen ist, spiegeln die Serumkreatininwerte also in signifikanter Weise den Zustand eines ANV wider. In unserer Studie diente diese Art der Analyse neben der Bestätigung bereits signifikanter Ergebnisse der Unterscheidung weiterer Parameter, die in den Gruppen I (NEV) und II (ANV/RIFLE) keine klaren Unterschiede aufwiesen, wie beispielsweise die Art der Pumpe, auf die im Punkt 5.2.2 eingegangen wird.

Ein weiterer Schwerpunkt der Untersuchungen dieser Arbeit wurde auf den postoperativen Blutverlust im Zusammenhang mit dem ANV gelegt. Dabei wurde nicht nur eine signifikante Beziehung zwischen dem absoluten Blutverlust in ml und dem Auftreten eines ANV mit und ohne NEV und dem absoluten Kreatininanstieg festgestellt, sondern auch in einer longitudinalen Analyse die Zeitverläufe des Blutverlustes in den Gruppen untersucht. Es stellte sich heraus, dass Patienten, die ein NEV benötigten, signifikant mehr Blut verloren, über einen längeren Zeitraum Blut verloren und einen signifikant anderen Zeitverlauf des Blutverlustes hatten als Patienten ohne Bedarf an NEV. Dass der Blutverlust in Folge des 
NEV entsteht, ist in diesem Außmaß eher unwahrscheinlich und es ist deshalb zu vermuten, dass der Blutverlust bzw. die dazu führenden intra- und postoperativen Konditionen die Entstehung eines ANV begünstigen und /oder verlängern könnten. In der Literatur sind diese Zusammenhänge nicht über den Zeitverlauf untersucht worden, sondern nur den absoluten Blutverlust betreffend. Landoni et al. (2006) beschreiben in ihrer 7846 Patienten umfassenden Studie einen signifikanten Unterschied zwischen Patienten, die ein NEV benötigten und allen anderen hinsichtlich eines Blutverlustes über $1000 \mathrm{ml}$. Inwieweit die Blutverluste neben den hämodynamischen Konsequenzen weitere, das ANV begünstigende Auswirkungen haben, muss in weiteren Studien überprüft werden. Die Tatsache, dass sich die Zeitverläufe, Zeiteffekte und absoluten Mengen der Blutverluste zwischen den Gruppen so signifikant unterscheiden, lässt vermuten, dass direkte Zusammenhänge vorliegen, die noch nicht eindeutig geklärt sind und dementsprechend auch in der Literatur noch nicht ausreichend beschrieben werden. Möglicherweise spielt die intravasale Hämolyse und die Exkretion vermehrter Mengen von freiem Hämoglobin über die Nieren im Sinne einer Pigmentnephropathie eine entscheidende Rolle. Nicht nur durch intravasale Hämolyse anfallendes freies Hämoglobin, sondern auch im Rahmen der Blutverluste verabreichte Transfusionen mit einem hohen Anteil an Hämoglobin und Zellbestandteilen könnte diesen Effekt verstärken. Zusammenfassend lässt sich sagen, dass postoperative Blutverluste in hohem Maße mit einem Nierenversagen assoziiert sind. Eine suffiziente Blutstillung sowie ein optimiertes Gerinnungsmanagement sind daher essentiell.

\subsubsection{HLM-assoziierte Parameter}

Der Einfluss der Herz-Lungen-Maschine auf das Auftreten eines postoperativen ANV wird in der Literatur sehr kontrovers diskutiert. Einige Autoren sehen durch die Manipulation an HLM-Parametern keine deutlichen Verbesserungen hinsichtlich der Entstehung eines ANV wie beispielsweise Garwood et al. (2004). Auch Lema et al. (1995) finden in ihrer Untersuchung keine negativen Effekte der Behandlung mit HLM auf die Nierenfunktion, allerdings nur für vorher nierengesunde Patienten. Sie kommen zu dem Ergebnis, dass nicht die Perfusionszeit selber, sondern die Vorgänge während der Operation vor und nach der Perfusionszeit die schädigenden Auswirkungen auf die Niere verursachen. Demgegenüber beschreibt die absolute Mehrzahl der Studien aber einen signifikanten Zusammenhang zwischen dem Auftreten eines ANV und der Dauer der Perfusionszeit. Ascione et al. (1999), Beauford et al. (2004), Fischer et al. (2002) und Landoni et al. (2006) belegen eindrucksvoll die Auswirkungen der HLM auf die Nierenfunktion, wie später genauer dargestellt wird. Einige Studien (Rastan et al. 2006, Ascione et al. 1999, Beauford et al. 2004) beschreiben 
zudem für bestimmte Anforderungen in der Revaskularisierungschirurgie einen Vorteil für am schlagenden Herzen operierte Patienten. Allerdings gibt es auch Studien, die diesen Vorteil nicht bestätigen können und eine Überlegenheit von Operationen am schlagenden Herzen wird zunehmend kritisch gesehen.

Trotz der negativen Auswirkungen der extrakorporalen Zirkulation auf den Organismus und die Nierenfunktion sind eine Großzahl der Operationen am Herzen nur mit Hilfe dieser Technik durchführbar, da die normale Herztätigkeit- bevor irreversible Schäden am Gehirn auftreten- höchstens 3 Minuten unterbrochen werden kann, die meisten Operationen am Herzen aber wesentlich länger dauern. Daher ist die Identifizierung von verbesserungswürdigen, mit der HLM in Beziehung stehenden Einflussgrößen wichtig, um die durch die extrakorporale Zirkulation ausgelösten schädigenden Auswirkungen auf die Niere möglichst zu minimieren.

In der vorliegenden Arbeit haben wir die gesamte Operationsdauer, aber auch die einzelnen Zeitabschnitte wie Ischämiezeit des Herzens, Perfusionszeit bzw. kardiopulmonale Bypasszeit und die Reperfusionszeit untersucht. In unserer Studie sind die gesamte Operationsdauer sowie alle weiteren Zeitphasen signifikant mit dem Auftreten eines ANV sowohl im Sinne eines NEV als auch nach RIFLE und dem absoluten Kreatininanstieg assoziiert. Bei der Untersuchung der Patienten mit NEV hatten Patienten ohne NEV im Median eine Operationsdauer von 270 min und solche mit NEV 335 min, was einem p-Wert von $<0,01$ entspricht. Die Ischämiezeit zeigte mit 78 min ohne NEV und 94 min mit NEV ein ebenfalls signifikantes Ergebnis. Auch die Perfusionszeit und Reperfusionszeit waren mit Zeiten für den Median von 124 min bzw. 37 min in der Gruppe ohne NEV und 168 min bzw. 50 min in der Gruppe mit NEV hochsignifikant verschieden. Patienten der Gruppe II (ANV gemäß RIFLE) hatten eine mediane Operationszeit von 308,5 min im Vergleich zu Patienten ohne ANV mit 264,0 min. Auch hier errechnet sich ein $\mathrm{p}$-Wert von $<0,01$. Die medianen Werte der Ischämiezeit, Perfusionszeit und Reperfusionszeit von Gruppe II im Vergleich zu den restlichen Patienten waren 88/75 min, 143/120 min und 43/36 min. Bei diesen Ergebnissen ist zu berücksichtigen, dass einige der untersuchten Zeiten sich überschneiden. Die gesamte Operationsdauer beispielsweise beinhaltet natürlich auch die Perfusionszeit. Da die Perfusionszeit an sich signifikante Auswirkungen auf die Entstehung eines ANV hat, ist nicht klar, ob der signifikante Effekt bei der gesamten Operationszeit nur von dem Anteil der Perfusionszeit herrührt, oder noch andere Faktoren während der restlichen Operationszeit eine Rolle spielen. Bei der Ischämiezeit des Herzens sind neben dem Zeitfaktor natürlich auch der 
Grad der Kühlung und die Art der Kardioplegie als Einflussfaktoren zu berücksichtigen. Die Perfusionszeit ist die Zeit, in der die Herz-Lungen-Maschine läuft, also die Funktion von Herz und Lunge ersetzt, wobei die Herz-Lungen-Maschine in der Anfangs- und Endphase der Operation partiell auch parallel zum schlagenden Herzen läuft. Dabei wird im ersten Schritt das Blut im Oxigenator oxygeniert, von der in den meisten Fällen verwendeten Rollenpumpe transportiert und im Wärmeaustauscher nach Bedarf temperiert. Dann werden die Gefäße abgeklemmt und die HLM übernimmt die Pumparbeit. Ebenso setzt nach dem Wiedereröffnen der Aortenklemme gegen Ende der Prozedur zwar die Koronardurchblutung wieder ein, aber die HLM wird nicht einfach ausgeschaltet, sondern langsam heruntergefahren, was man als partiellen Bypass bezeichnet, bis die Herzfunktion bzw. Pumpleistung und Hämodynamik wieder ausreichend für eine eigenständige Versorgung sind. In der Literatur wird oft von dieser cross clamp- Zeit gesprochen und in den meisten Studien (Bahar et al. 2005, Conlon et al. 1999, Heikkinen et al. 1986, Khiji et al. 2004, Mangos et al. 1995, Grayson et al. 2003) wurde sie mit der Entstehung eines ANV in signifikanten Zusammenhang gebracht. Kumbhani et al. (2005) stellten diesen Zusammenhang zwischen dem ph-Wert im Myokard während der cross-clamp- Zeit und dem Überleben bzw. dem Auftreten von Komplikationen her. Eine regionale myokardiale Azidose war dabei hoch signifikant mit einem niedrigeren Überleben von 30 Tagen postoperativ assoziiert. In dieser Studie von Kumbhanie et al. (2005) wurden 398 Patienten untersucht. Badner et al. (1992) beschrieben in ihrer Studie mit 100 Patienten aber, dass das Management des ph-Werts keinen signifikanten Einfluss auf die Entstehung eines postoperativen ANV habe. Derart kontroverse Ansichten bestehen auch bezüglich des Effektes der HLM an sich. Obwohl Beauford et al. (2004) in ihrer Studie bei Operationen mit und ohne HLM signifikante Unterschiede bezüglich der Inzidenz eines ANV von $17 \%$ vs $9 \%$ und eines NEV von $10 \%$ vs. $3 \%$ und einer niedrigeren Mortalität feststellen, beschreiben Chukwuemeka et al. (2005), dass die Operation am schlagenden Herzen das Risiko nicht erhöht, ein ANV im Sinne eines Abfalls der Kreatinin-Clearance unter $60 \mathrm{ml} / \mathrm{min}$ zu entwickeln. In den meisten Studien wurden also die Perfusionszeit (siehe oben) oder die Ischämiezeit (Bahar et al. 2005, Khiji et al. 2004) untersucht. Die anderen Zeiten wurden kaum analysiert, obwohl die Arbeit von Lema et al. (1995) darauf hindeutet, dass die Periode vor und nach dem kompletten Bypass auf die Nierenfunktion schädigendere Auswirkungen haben könnte als die Bypasszeit selber. Lema et al. (1995) untersuchten anhand der glomerulären Filtration, errechnet aus der InsulinClearance und dem effektiven renalen Plasmafluss, abgeschätzt aus der ${ }^{125}$ I-HippuranClearance, die Auswirkungen der einzelnen Operationsphasen auf die Nierenfunktion. Aus 
den Ergebnissen folgerten sie, dass die Nierenfunktion bei zuvor nierengesunden Patienten nicht negativ durch die HLM beeinflusst wird, sondern eher durch die Phasen vor und nach der Perfusionszeit, was dem partiellen Bypass während der Kühlung und dem partiellen Bypass nach der Öffnung der Aortenklemme entsprechen würde. Mit unseren Ergebnissen können wir jedenfalls bestätigen, dass die Länge der Reperfusionszeit neben Ischämiezeit und Perfusionszeit ebenfalls signifikant mit der Entstehung des ANV assoziiert ist. Es zeigt sich also auch an den hier erhobenen Daten, entgegen den Ergebnissen von Lema et al. (1995), welche allerdings auch nur 12 Patienten untersuchten, ein hochsignifikanter Unterschied zwischen den Gruppen mit und ohne akutes Nierenversagen bezüglich der Perfusionszeit. Man muss daraus schließen, dass die extrakorporale Zirkulation negative Auswirkungen auf die Nierenfunktion hat. Bekräftigt wird diese Hypothese durch Arbeiten wie die von Boldt et al. (2003) oder Fischer et al. (2002). Boldt et al. fanden 2003 anhand der Messung spezifischer, eine Tubulusschädigung anzeigender Enzyme (N-acetyl-beta-DGlucosaminidase, alpha 1-Microglobulin, Glutathiontransferase, Glutathiontransferase-alpha) heraus, dass die HLM und deren Dauer eine Tubulusschädigung bewirkten. Dabei wählten sie einen Trennwert und stellten signifikant unterschiedliche Auswirkungen zwischen HLMZeiten unter 70 min und solchen über 90 min fest. Der modifizierte Primärharn gibt Hinweise auf eine zunehmende Tubulusinsuffizienz in Analogie zur Perfusionszeit. Mit zunehmender Perfusionszeit nimmt auch die Hämolyse zu, und ansteigende Mengen von freiem Hämoglobin führen verstärkt zu einer Pigmentnephropathie. Der kumulative Effekt dieser pathopyhsiologischen Vorgänge könnte zumindestens teilweise den Zusammenhang zwischen langen Perfusionszeiten und vermehrt auftretendem akuten Nierenversagen erklären. In der Studie von Fischer et al. (2002) wurde ebenfalls ein eindeutiger, schädigender Einfluss der HLM auf die Nierenfunktion analysiert. Bei 143 Patienten wurde in der Gruppe mit ANV eine längere Perfusionszeit, geringere HLM-Flussraten und längere Perioden mit Flüssen unter $60 \mathrm{~mm} \mathrm{Hg}$ Druck festgestellt.

Bei der Wahl der Pumpe der HLM und der Kardioplegielösung konnten in unserer Arbeit keine signifikanten Unterschiede zwischen einer Zentrifugalpumpe und einer Rollerpumpe und der Blutkardioplegie und der Kardioplegie nach Bretschneider bezüglich des Auftretens eines ANV herausgefunden werden. Was allerdings die Untersuchung des absoluten Kreatininanstiegs betrifft, hat sich herausgestellt, dass die Rollerpumpe einen weniger hohen Kreatininanstieg mit sich brachte als die Zentrifugalpumpe. Der Zusammenhang war mit $\mathrm{p}=$ 0,02 signifikant. Auch hier könnte eine durch die Scherkräfte in unterschiedlichem Grad ausgelöste Hämolyse, gepaart mit der systemischen Inflammationsreaktion des Körpers, eine 
Rolle spielen. Bei dem quasi pulsatilen Fluss der Rollerpumpe scheinen diese negativen Auswirkungen geringer zu sein.

Bei der Wahl der Kardioplegielösung war die Lösung nach Bretschneider mit einem signifikant niedrigeren Anstieg der Kreatininwerte assoziiert. Hier war der p-Wert 0,01. Die Unterschiede sind also präsent, aber nicht stark genug, um sich in dem Bedarf eines NEV oder der Definition eines ANV nach RIFLE widerzuspiegeln. Trotzdem sollten sie als Hinweis auf Unterschiede zwischen den Gruppe gewertet werden und ein Vorteil der Rollerpumpe gegenüber der Zentrifugalpumpe und der Kardioplegie nach Bretschneider gegenüber der Blutkardioplegie ist zu vermuten. In der Literatur wurde anhand von Tierversuchen untersucht, inwieweit eine pulsatile Perfusion die Nierenfunktion schonen kann. Singal et al. (2006) untersuchten an Schweinen anhand der Messung von Gammaglutamyltranspeptidase, Glutathion S-Transferase und Alkalischer Phophatase als renalen Enzymen den Einfluss einer biologisch-variablen Perfusion mit einem Computersystem für eine physiologische Schlag zu Schlag Variabilität einer Rollerpumpe im Vergleich zur nonpulsatiler Perfusion. Dabei stellte sich heraus, dass die Marker für eine tubuläre Schädigung bei nonpulsatilen Perfusion signifikant erhöht waren und die Urinausscheidung bei der Schlag-zu-Schlag-Perfusion besser war. Außerdem konnte die Hypothermiedauer bzw. die als schädlich vermutete Phase der Abkühlung bzw. Wiederaufwärmung verkürzt werden.

Die systemische Hypothermie während der Herzoperation wird durch temperiertes Wasser, welches die blutleitenden Röhren der HLM umfließt, erreicht und dient der Senkung der Stoffwechselaktivität und des Sauerstoffverbrauchs des ganzen Organismus. Die Applikation von kalter Kardioplegieslösung in die Koronargefäße zählt zu den kardioprotektiven Maßnahmen und dient der Erhöhung der Ischämietoleranz der Herzmuskelzellen, welche ohne derartige Maßnahmen nur ca. 15 min betrüge (Harrison et al. 2005). In der vorliegenden Arbeit konnte sowohl die minimale Temperatur mit der Entstehung eines ANV im Sinne der Notwendigkeit eines Nierenersatzverfahrens in Verbindung gebracht werden als auch eine signifikant unterschiedliche Auswirkung dreier Gruppen der Temperatur, nämlich der Normothermie $\left(34-37^{\circ} \mathrm{C}\right)$, der milden Hypothermie $\left(28-33^{\circ} \mathrm{C}\right)$ und der tiefen Hypothermie $\left(18-27^{\circ} \mathrm{C}\right)$. Eine tiefe Hypothermie wurde bei langen und schwierigen Eingriffen zum Einsatz gebracht, und ist somit unter Umständen mit weiteren Risikofaktoren assoziiert, welche nicht eindeutig zu isolieren sind. Nur 8\% der Patienten mit einer Normothermie benötigten ein postoperatives NEV im Vergleich zu 13\% mit milder Hypothermie und 26\% mit tiefer 
Hypothermie. Dies entspricht p-Werten von jeweils unter 0,01. Beim ANV nach RIFLEKriterien zeigte sich ein ähnliches Muster. 30\% der Patienten mit Normothermie entwickelten ein solches ANV im Vergleich zu 36\% der Patienten mit milder Hypothermie und 63\% der Patienten mit tiefer Hypothermie. Die niedrige Temperatur ist also im Bezug auf ein ANV als Risikofaktor zu bewerten. Zu dieser Ansicht kamen auch Bahar et al. (2005) in ihrer 14437 Patienten umfassenden Studie. Auch Singal et al. (2006) gehen in ihrer Studie davon aus, dass eine verkürzte Hypothermiedauer nephroprotektiv wirkt. Inwieweit die systemische Hypothermie unabhängig die Nierenfunktion negativ beeinflusst, muss in weiteren Studien geklärt werden, um eine optimale Temperatur für die systemische Kühlung und gleichermaßen die Protektion der Niere zu eruieren. 


\section{Zusammenfassung}

Das akute Nierenversagen nach kardiochirurgischen Operationen ist mit weitreichenden Komplikationen assoziiert, welche oft nur schwierig und kostenintensiv zu behandeln sind und bringt eine hohe Mortalität mit sich, die meist 60\% und mehr beträgt (Garwood et al. 2004). Obwohl technische und organisatorische Verbesserungen durchgeführt wurden, wie die Revaskularisierungsoperationen am schlagenden Herzen und die Einführung biokompatibler Dialysemembranen, konnte das Outcome ( = Auskommen) der Patienten kaum verbessert werden (Garwood et al. 2004). Neben dem steigenden Alter der kardiochirurgischen Patienten und den Vorerkrankungen, die dieses mit sich bringt, könnte die komplexe Pathophysiologie des ANV und seine Bedeutung im Sinne eines multifaktoriellen Geschehens zu dieser Tatsache beitragen.

In der vorliegenden Arbeit wurde untersucht, wie perioperative Parameter mit dem Auftreten eines ANV assoziiert sind. Zu diesem Zweck wurden in einer retrospektiven Analyse die Daten von 3574 Patienten, welche sich in den Jahren 2000 bis 2005 einer Herzoperation in der Klinik für Thorax-, Herz- und Gefäßchirurgie des Universitätsklinikums Göttingen unterziehen mussten, erhoben, archiviert und analysiert. Nach Erstellung eines gemeinsamen Datensatztes aus den Datenbanken der verschiedenen Intensivstationen und der Herz-LungenMaschine konnten auch intraoperative HLM-assoziierte Parameter mit dem Auftreten eines ANV, dessen Definition anhand der Daten aus den Intensivstationen möglich war, verglichen werden. Um die Ergebnisse der Studie mit der Literatur vergleichen zu können und trotzdem dem neuesten Stand der Forschung gerecht zu werden, wurde das ANV in zwei Gruppen definiert. In der Gruppe I war ein ANV durch den Einsatz eines Nierenersatzverfahrens (NEV) und in der Gruppe II durch die international anerkannten Kriterien nach RIFLE (Venkataraman et Kellum 2007) definiert (erhöhtes Serumkreatinin um Faktor 3 oder Abfall der GFR um 75\% oder Serumkreatininwert von $4 \mathrm{mg} / \mathrm{dl}$ ). Um weitere durch das Raster dieser Definitionen fallende Unterschiede sichtbar zu machen, wurde zudem eine Analyse aller Parameter im Zusammenhang mit dem absoluten Serumkreatininanstieg in der postoperativen Phase vorgenommen. Auf diese Weise wurden das Alter, Geschlecht, Art der Operation, Reoperation, IABP, Operationsdauer, Ischämiezeit, Perfusionszeit, Reperfusionszeit, Art der Pumpe, Art der Kardioplegie, Blutverluste und der Grad der Hypothermie untersucht. Für alle Tests wurde als Signifikanzniveau $\alpha=5 \%$ festgelegt. 
Alle operierten Patienten waren zwischen 18 und 92 Jahren alt, wobei der Median des Alters bei 68 Jahren lag. 73\% der Patienten waren zwischen 60 und 80 Jahre alt. 71\% der Patienten waren Männer und 29\% Frauen. Die Art der Operationen unterteilte sich mit 61\% auf Revaskularisierungseingriffe, 20\% Herzklappeneingriffe, 15\% Kombinationseingriffe an Klappen und Herzkranzgefäßen und $4 \%$ anderer Operation, die nicht in diese Kategorien fielen. Dabei zeigte sich neben einer Gesamtmortalität von 6,4\% eine Inzidenz des ANV im Sinne eines NEV von 10\% und im Sinne der RIFLE-Kriterien von 32\%. Die Mortalität der Patienten mit NEV lag bei $48 \%$ und die der Patienten mit ANV bei $14 \%$.

Der überwiegende Teil der in unserer Studie untersuchten Einflussgrößen hatte eine signifikante Auswirkung auf die Entstehung eines ANV. Besonders hervorzuheben sind hier die Perfusionszeit, die Blutverluste, die Kombinations-, und Klappeneingriffe und die Hypothermie. Darüber hinaus war sowohl das Alter als auch das weibliche Geschlecht der Patienten signifikant mit dem Auftreten eines ANV verbunden. Ebenso waren eine Reoperation, Klappenoperation und der Einsatz einer IABP hoch signifikant mit dem Auftreten eines ANV verknüpft. Auch die jeweilige Länge der Operationszeit, Ischämiezeit, Perfusionszeit und Reperfusionszeit und die minimale Temperatur während der Operation waren Risikofaktoren für ein ANV. Die Patienten mit ANV hatten einen signifikant höheren und länger andauernden Blutverlust als Patienten ohne ANV. Bei all diesen Zusammenhängen konnte ein p-Wert von jeweils unter 0,01 errechnet werden. Die Art der Pumpe und die Art der Kardioplegie zeigten im Bezug auf das NEV und die Definition des ANV nach RIFLE keine signifikanten Unterschiede, allerdings führte die Zentrifugalpumpe zu einem signifikant höheren absoluten Kreatininanstieg als die Rollerpumpe. Ebenso war die Blutkardioplegie mit signifikant höheren Kreatininwerten assoziiert als die Kardioplegielösung nach Bretschneider. Die Inzidenzen und Mortalitätsraten in der vorliegenden Studie liegen innerhalb der Bandbreite der in der Literatur angegebenen Maßzahlen. Die Mortalität der Patienten mit NEV liegt in unserer Studie mit $48 \%$ eher unterhalb des Niveaus der in der Literatur angegebenen Werte, die meist bei 50-60\% liegen (Garwood et al. 2004). Die übrigen Ergebnisse unserer Studie wie Alter, Geschlecht, Klappenoperation, Kombinationseingriffe, Blutverluste, Hypothermie, Op-Zeit, Ischämiezeit und Perfusionszeit wurden in der großen Mehrzahl durch weitere Studien bestätigt, welche zu gleichen oder ähnlichen Ergebnissen kamen.

Welche Phase der Operation nun den entscheidenden schädigenden Einfluss auf die Niere hat, der partielle Bypass vor dem Abklemmen der Gefäße während der Abkühlung, der 
kardiopulmonale Bypass selber oder der partielle Bypass nach Freigabe der Koronarperfusion durch Lösen der Aortenklemme, bleibt noch ungewiss und muss in weiteren Studien erörtert werden. In unserer Studie belegen aber alle einzelnen Zeitabschnitte während der Herzoperation einen Zusammenhang mit der Entstehung eines ANV. Auch die Frage inwieweit eine Hypothermie Nutzen für den Gesamtorganismus aber auch Schaden für die Nierenfunktion darstellt scheint eine Gratwanderung zu sein und bedarf noch weiterer Klärung. In der vorliegenden Arbeit fanden wir auch einen sehr signifikanten Zusammenhang zwischen den Blutverlusten und deren Zeitverläufen und dem Auftreten eines ANV. In welchem Maße hierbei neben dem Volumenverlust unter hämodynamischen Gesichtspunkten weitere Mechanismen ein Rolle spielen, ist eine interessante, in weiteren Studien zu untersuchende Fragestellung. 


\section{Literaturverzeichnis}

Abu-Omar Y, Ratnatunga C (2006): Cardiopulmonary bypass and renal injury. Perfusion $\underline{21}$ (4), 209-213

Andersson LG, Ekroth R, Bratteby LE, Hallhagen S, Wesslén O (1993): Acute renal failure after coronary surgery - a study of incidence and risk factors in 2009 consecutive patients. Thorac Cardiovasc Surg $\underline{41}$ (4), 237-241

Andrews PM, Coffey AK (1983): Cytoplasmic contractile elements in glomerular cells. Fed Proc $\underline{42}$ (14), 3046-3052

Ascione R, Lloyd CT, Underwood MJ, Gomes WJ, Angelini GD (1999): On-pump versus off-pump coronary revascularization: evaluation of renal function. Ann Thorac Surg $\underline{68}$ (2), 493-498

Badner NH, Murkin JM, Lok P (1992): Differences in pH management and pulsatile/nonpulsatile perfusion during cardiopulmonary bypass do not influence renal function. Anesth Analg $\underline{75}$ (5), 696-701

Badr KF, Ichikawa I (1988): Prerenal failure: a deleterious shift from renal compensation to decompensation. N Engl J Med 3198 (10), 623-629

Bahar I, Akgul A, Ozatik MA, Vural KM, Demirbag AE, Boran M, Tasdemir O (2005): Acute renal failure following open heart surgery: risk factors and prognosis. Perfusion 20 (6), 317-322

Baylis C, Rennke HR, Brenner BM (1977): Mechanisms of the defect in glomerular ultrafiltration associated with gentamicin administration. Kidney Int $\underline{12}$ (5), 344-453

Beauford RB, Saunders CR, Niemeier LA, Lunceford TA, Karanam R, Prendergast T, Shah S, Burns P, Sardari F, Goldstein DJ (2004): Is off-pump revascularization better for patients with non-dialysis-dependent renal insufficiency? Heart Surg Forum $\underline{7}$ (2), E141-146 
Bellomo R, Farmer M, Bhonagiri S, Porceddu S, Ariens M, M'Pisi D, Ronco C (1999): Changing acute renal failure treatment from intermittent hemodialysis to continuous hemofiltration: impact on azotemic control. Int J Artif Organs 22 (3), 145-150

Bellomo R, Chapman M, Finfer S, Hickling K, Myburgh J (2000): Low-dose dopamine in patients with early renal dysfunction: a placebo-controlled randomised trial. Australian and New Zealand Intensive Care Society (ANZICS) Clinical Trials Group. Lancet 356 (9248), 2139-2143

Bellomo R, Raman J, Ronco C (2001): Intensive care unit management of the critically ill patient with fluid overload after open heart surgery. Cardiology $\underline{96}$ (3-4), 169-176

Boldt J, Brenner T, Lehmann A, Suttner SW, Kumle B, Isgro F (2003): Is kidney function altered by the duration of cardiopulmonary bypass? Ann Thorac Surg $\underline{75}$ (3), 906-912

Brenner BM, Troy JL, Daugharty TM, Deen WM, Robertson CR (1972): Dynamics of glomerular ultrafiltration in the rat. II. Plasma-flow dependence of GFR. Am J Physiol 223 (5), 1184-1190

Brezis M, Rosen S (1995): Hypoxia of the renal medulla--its implications for disease. N Engl J Med $\underline{332}$ (10), 647-655

Brunner E, Domhof S, Langer F: Nonparametric Analysis of Longitudinal Data in Factorial Experiments.Wiley Series, New York 2002.

Burckhardt G: Prof. Dr. med. Gerhard Burckhardt, Abteilung vegetative Physiologie und Pathophysiologie, Georg-August-Universität Göttingen, Humboldtallee 23, 37077 Göttingen, Vorlesungen, Unterrichtsmaterialien, Skripte, persönliche Beratung

Chertow GM, Levy EM, Hammermeister KE, Grover F, Daley J (1998): Independent association between acute renal failure and mortality following cardiac surgery. Am J Med $\underline{104}(4), 343-348$ 
Chukwuemeka A, Weisel A, Maganti M, Nette AF, Wijeysundera DN, Beattie WS, Borger MA (2005): Renal dysfunction in high-risk patients after on-pump and off-pump coronary artery bypass surgery: a propensity score analysis. Ann Thorac Surg $\underline{80}$ (6), 2148-2153

Conlon PJ, Stafford-Smith M, White WD, Newman MF, King S, Winn MP, Landolfo K. (1999): Acute renal failure following cardiac surgery. Nephrol Dial Transplant 14 (5), 11581162

Fischer UM, Weissenberger WK, Warters RD, Geissler HJ, Allen SJ, Mehlhorn U (2002): Impact of cardiopulmonary bypass management on postcardiac surgery renal function. Perfusion $\underline{17}$ (6), 401-406

Frost L, Lund O, Pedersen RS, Hansen OK, Hansen HE (1992): Prognosis and risk factors in acute dialysis-requiring renal failure after open-heart surgery. Ugeskr Laeger 154 (37), 24812485

Galle J, Wanner C (1996): Impact of nitric oxide on renal hemodynamics and glomerular function: modulation by atherogenic lipoproteins? Kidney Blood Press Res $\underline{19}$, 2-15

Garwood S (2004): Renal insufficiency after cardiac surgery. Semin Cardiothorac Vasc Anesth $\underline{8}(3), 227-241$

Grayson AD, Khater M, Jackson M, Fox MA (2003): Valvular heart operation is an independent risk factor for acute renal failure. Ann Thorac Surg $\underline{75}$ (3), 1829-1835

Harris PJ, Navar LG (1985): Tubular transport responses to angiotensin. Am J Physiol 248 (5 PT 2), F621-630

Harrison T.R: Harrisons Innere Medizin - Deutsche Ausgabe in Zusammenarbeit mit der Charité. 16. Auflage; hrsg. v. Dietel M, Suttorp N, Zeitz M; ABW Wissenschaftsverlag, Berlin 2005, 1755-1788 
Heikkinen L, Harjula A, Merikallio E (1985): Acute renal failure related to open-heart surgery. Ann Chir Gynaecol 74 (5), 203-209

Hörl W: Dialyseverfahren in Klinik und Praxis - Technik und Klinik. 6.Auflage; hrsg. v. Hörl W, Wanner C; Georg Thieme Verlag, Stuttgart,New York 2004, 11-115

Joannidis M, Bonn G, Pfaller W (1989): Lipid peroxidation--an initial event in experimental acute renal failure. Ren Physiol Biochem 12 (1), 47-55

Joannidis M, Gstraunthaler G, Pfaller W (1990): Xanthine oxidase: evidence against a causative role in renal reperfusion injury. Am J Physiol 258 (2 PT 2), F232-6

Joannidis M, Spokes K, Nakamura T, Faletto D, Cantley LG (1994): Regional expression of hepatocyte growth factor/c-met in experimental renal hypertrophy and hyperplasia. Am J Physiol 267 (2 PT 2), F231-6

Johnson PC (1986): Autoregulation of blood flow. Circ Res 59 (5), 483-95

Junqueira LC, Carneiro J, Kelley RO: Histologie. 5. Auflage; hrsg. v. Gratzl M; Springer Verlag, 2002, 329

Khilji SA, Khan AH (2004): Acute renal failure after cardiopulmonary bypass surgery. J Ayub Med Coll, Abbottabad 16 (3), 25-28

Krautzig S: Basislehrbuch Innere Medizin. 3. Auflage; hrsg. v. Renz-Polster H, Krautzig S, Braun J; Urban Fischer Verlag, München 2004, 878-940

Kumbhani DJ, Healey NA, Biswas KS, Birjiniuk V, Crittenden MD, Treanor PR, Khuri SF (2005): Adverse 30-day outcomes after cardiac surgery: predictive role of intraoperative myocardial acidosis. Ann Thorac Surg $\underline{80}$ (5), 1751-1757

Lameire N, Vanholder R: Acute renal failure: pathophysiology and prevention. In: Oxford Textbook of Clinical Nephrology. Hrsg. v. Davison AM, Cameron JS, Grünfeld JP, Kerr DNS, Ritz E, Winearls CG. $2^{\text {nd }}$ edition; Oxford University Press, 1998, 1197-1260 
Landoni G, Zangrillo A, Franco A, Aletti G, Roberti A, Calabrò MG, Slaviero G, Bignami E, Marino G (2006): Long-term outcome of patients who require renal replacement therapy after cardiac surgery. Eur J Anaesthesiol $\underline{23}$ (1), 17-22

Lema G, Meneses G, Urzua J, Jalil R, Canessa R, Moran S, Irarrazaval MJ, Zalaquett R, Orellana P (1995): Effects of extracorporeal circulation on renal function in coronary surgical patients. Anesth Analg $\underline{81}$ (3), 446-451

Leonard H (1992): Spezielle Anatomie 2. Eingeweide-Nervensystem-Systematik der Muskeln und Leitungsbahnen. Band 2: Taschenlehrbuch der gesamten Anatomie; hrsg. v. Frick H, Leonhardt H, Starck D unter Mitarbeit namhafter Autoren; Georg Thieme Verlag, Stuttgart,-New York 1992, 149-161

Mangos GJ, Brown MA, Chan WY, Horton D, Trew P, Whitworth JA (1995): Acute renal failure following cardiac surgery: incidence, outcomes and risk factors. Aust N Z J Med $2 \underline{5}$ (4), 284-249

Noyez L, Plesiewicz I, Verheugt FW (2006): Estimated creatinine clearance instead of plasma creatinine level as prognostic test for postoperative renal function in patients undergoing coronary artery bypass surgery. Eur J Cardiothorac Surg 29 (4), 461-465

Pschyrembel W: Pschyrembel - Klinisches Wörterbuch. 259. Auflage; Walter de Gruyter Verlag, Berlin,New York 2002, 1341

Rastan AJ, Eckenstein JI, Hentschel B, Funkat AK, Gummert JF, Doll N, Walther T, Falk V, Mohr FW (2006): Emergency coronary artery bypass graft surgery for acute coronary syndrome: beating heart versus conventional cardioplegic cardiac arrest strategies. Circulation $\underline{114}$ (1 suppl), 1477-1485 
Ronco C, Bellomo R, Brendolan A, Pinna V, La Greca G (1999): Brain density changes during renal replacement in critically ill patients with acute renal failure. Continuous hemofiltration versus intermittent hemodialysis. J Nephrol $\underline{12}$ (3), 173-178

Silbernagl S: Taschenatlas der Pathophysiologie; hrsg. v. Silbernagel S, Lang F; Georg Thieme Verlag, Stuttgart,New York 1998, 92-132

Silbernagl S: Taschenatlas der Physiologie. 6. Auflage; hrsg. v. Silbernagel S, Despopoulos A; Georg Thieme Verlag, Stuttgart,New York 2003, 148-184

Simon C, Luciani R, Capuano F, Miceli A, Roscitano A, Tonelli E, Sinatra R (2007): Mild and moderate renal dysfunction: impact on short-term outcome. Eur J Cardiothorac Surg $\underline{32}$ (2), 286-290

Singal RK, Docking LM, Girling LG, Graham MR, Nickerson PW, McManus BM, Magil AB, Walker EK, Warrian RK, Cheang MS, Mutch WA (2006): Biologically variable bypass reduces enzymuria after deep hypothermic circulatory arrest. Ann Thorac Surg $\underline{82}$ (4), 14801488

Thakar CV, Liangos O, Yared JP, Nelson D, Piedmonte MR, Hariachar S, Paganini EP (2003): ARF after open-heart surgery: Influence of gender and race. Am J Kidney Dis 41 (4), 742-751

Thakar CV, Arrigain S, Worley S, Yared JP, Paganini EP (2005): A clinical score to predict acute renal failure after cardiac surgery. J Am Soc Nephrol $\underline{16}$ (1), 162-168

Venkataraman R, Kellum JA (2007): Defining acute renal failure: the RIFLE criteria. J Intensive Care Med 22 (4), 187-193

Wijeysundera DN, Karkouti K, Beattie WS, Rao V, Ivanov J (2006): Improving the identification of patients at risk of postoperative renal failure after cardiac surgery. Anesthesiology 104 (1), 65-72 


\section{Anhang}

\subsection{Tabellenverzeichnis}

\begin{tabular}{|c|c|c|}
\hline Tabelle Nr. & Titel der Tabelle & Seite \\
\hline Tab.1 & RIFLE-Kriterien & 8 \\
\hline Tab.2 & Art des Nierenversagens & 11 \\
\hline Tab.3 & Urinindizes & 22 \\
\hline Tab.4 & Fraktionelle Natriumausscheidung & 23 \\
\hline Tab.5 & Dialyse und Hämofiltrationsverfahren & 30 \\
\hline Tab.6 & Gruppeneinteilung des ANV & 39 \\
\hline Tab.7 & Datensatzentstehung & 41 \\
\hline Tab.8 & kategorielle Parameter und NEV & 51 \\
\hline Tab.9 & metrische Parameter und NEV & 56 \\
\hline Tab.10 & Blutverluste über die Zeit & 60 \\
\hline Tab.11 & kategorielle Parameter und ANV & 62 \\
\hline Tab.12 & metrische Parameter und ANV & 66 \\
\hline Tab.13 & Kreatinin, kategorielle Parameter & 69 \\
\hline Tab.14 & Kreatinin, metrische Parameter & 70 \\
\hline Tab.15 & Zusammenfassung Ergebnisse & 71 \\
\hline
\end{tabular}




\subsection{Abbildungsverzeichnis}

\begin{tabular}{|c|c|c|}
\hline Abbildungs $\mathrm{Nr}$. & Titel der Abbildung & Seite \\
\hline Abb.1 & Aufbau des Glomerulus & 3 \\
\hline Abb.2 & Nephron & 5 \\
\hline Abb.3 & Übersicht über die Patientengruppe & 37 \\
\hline Abb.4 & Geschlechterverteilung & 45 \\
\hline Abb.5 & Histogramm der Altersverteilung & 46 \\
\hline Abb.6 & Box-Plot Altersverteilung & 47 \\
\hline Abb.7 & Art der Operation & 48 \\
\hline Abb. 8 & Einsatz von NEV & 49 \\
\hline Abb.9 & Häufigkeit des ANV gemäß RIFLE-Kriterien & 50 \\
\hline Abb.10 & Geschlecht und NEV & 52 \\
\hline Abb.11 & Klappenoperationen und NEV & 53 \\
\hline Abb. 12 & Art der Operation und NEV & 54 \\
\hline Abb.13 & Temperatur und NEV & 55 \\
\hline Abb.14 & Alter und NEV & 56 \\
\hline Abb. 15 & Operationsdauer und NEV & 57 \\
\hline Abb.16 & Ischämiezeit und NEV & 57 \\
\hline Abb.17 & Perfusionszeit und NEV & 58 \\
\hline Abb.18 & Reperfusionszeit und NEV & 58 \\
\hline Abb. 19 & Niedrigste Temperatur und NEV & 58 \\
\hline Abb. 20 & Blutverluste über die Zeit (Median) & 59 \\
\hline Abb.21 & Blutverluste (50\%- \& 75\%-Quantil) & 61 \\
\hline Abb.22 & IABP und ANV & 63 \\
\hline Abb.23 & Art der Operation und ANV & 64 \\
\hline Abb.24 & Temperatur und ANV & 65 \\
\hline Abb. 25 & Alter und ANV & 66 \\
\hline Abb.26 & Ischämiezeit und ANV & 67 \\
\hline Abb. 27 & Perfusionszeit und ANV & 67 \\
\hline Abb.28 & Reperfusionszeit und ANV & 67 \\
\hline Abb.29 & Minimale Temperatur und ANV & 67 \\
\hline Abb.30 & Blutverluste und ANV & 68 \\
\hline Abb.31 & Ischämiezeit und Kreatinin & 70 \\
\hline Abb. 32 & Min.Temperatur und Kreatinin & 70 \\
\hline Abb. 33 & Blutverlust und Kreatinin & 70 \\
\hline
\end{tabular}




\subsection{Abkürzungsverzeichnis}

\begin{tabular}{|c|c|}
\hline ANV & akutes Nierenversagen \\
\hline $\mathrm{ADH}$ & antidiuretisches Hormon \\
\hline ANP & atrial natriuretic peptide \\
\hline ATN & akute Tubulusnekrose \\
\hline BNP & brain natriuretic peptide \\
\hline CAVH & kontinuierliche arteriovenöse Hämofiltration \\
\hline CAVHD & kontinuierliche arteriovenöse Hämodialyse \\
\hline COPD & chronic-obstructive-pulmonary-disease \\
\hline CSV & comma separated value \\
\hline $\mathrm{CVVH}$ & kontinuierliche venovenöse Hämofiltration \\
\hline CVVHD & kontinuierliche venovenöse Hämodialyse \\
\hline CVVHDF & kontinuierliche venovenöse Hämodiafiltration \\
\hline EDD & slow efficient daily dialysis \\
\hline EGF & epidermal-growth-factor \\
\hline EKG & Elektorkardiogramm \\
\hline EPO & Erythropoetin \\
\hline GFR & glomeruläre Filtrationsrate \\
\hline GISI & Göttinger Informationssystem für Intensivmedizin \\
\hline HGF & hepatocyte-growth-factor \\
\hline HLM & Herz-Lungen-Maschine \\
\hline $\mathrm{HZV}$ & Herz-Zeit-Volumen \\
\hline IABP & intraaortale Ballonpumpe \\
\hline IGF-1 & insulin-like-growth-factor \\
\hline $\mathrm{NaCl}$ & Natriumchlorid \\
\hline $\mathrm{NEV}$ & Nierenersatzverfahren \\
\hline NO & Stickodxid \\
\hline OP & Operation \\
\hline PAF & plättchenaktivierender Faktor \\
\hline SCUF & slow continuous ultrafiltration \\
\hline SIRS & systemic inflammatory response syndrome \\
\hline SLEDD & slow low-efficient daily dialysis \\
\hline SOP & standard operating procedure \\
\hline ZVD & zentraler Venendruck \\
\hline
\end{tabular}




\section{Danksagung}

An dieser Stelle möchte ich mich ganz herzlich bedanken bei:

Meinem Doktorvater, Prof. Dr. med. Dipl.- Phys. F.A. Schöndube, Leiter der Klinik für Thorax-, Herz- und Gefäßchirurgie des Universitätsklinikums Göttingen, für die Überlassung des Themas und die sehr gute sachliche und persönliche Zusammenarbeit

Meinem Betreuer bei dieser Arbeit, Dr. Martin Friedrich, Facharzt in der Klinik für Thorax,Herz- und Gefäßchirurgie des Universitätsklinikums Göttingen, für die Realisierung des gesamten Projektes sowie der ausdauernden und vorbildlichen menschlichen und fachlichen Betreuung auch in schwierigen Momenten der Entstehung dieser Studie

Dr. Thomas Renner, Informatiker im Klinikum Göttingen, für die großzügige Hilfe und Beratung in allen Fragen der Datengewinnung und Verwaltung

Elger Thiele, Informatiker, für die außerordentliche Kreativität und Kompetenz bei der Verwaltung und Erstellung der Datensätze

Dr. Klaus Jung, Statistiker in der Abteilung Medizinische Statistik in Göttingen, für die kompetente und flexible Hilfe und Betreuung bei der Statistischen Auswertung in dieser Arbeit

Dr. med Daniel Heise, Zentrum Anästhesiologie, Rettungs- und Intensivmedizin des Universitätsklinikums Göttingen, für die nette Beratung zu Fragen bezüglich des akuten Nierenversagens und dessen Definition

Dr. med Thorsten Perl und Reiner Wäschle, Zentrum für Anästhesiologie, Rettungs- und Intensivmedizin des Universitätsklinikums Göttingen, für die freundliche Unterstützung bei der Datensatzabfrage. 


\section{Lebenslauf}

Am 14.10.1978 wurde ich in München als Sohn von Karlheinz Maus und Rosemarie Maus geboren.

Meine Schulbildung erhielt ich in Niedersachsen, Nordrhein-Westfalen und Bayern, wo ich im Sommer 1999 in Schwabmünchen die Allgemeine Hochschulreife erwarb.

Von 1999 bis 2000 leistete ich meinen Wehrdienst, der mit der Ausbildung zum Sanitäter begann, im Bundeswehrkrankenhaus in Ulm in der stationären- und Op- Pflege ab. Während dieser Zeit wurde ich zur Vertauensperson aller Wehrdienstleistender am Bundeswehrkrankenhaus in Ulm gewählt.

Nach einem Auslandsaufenthalt in Frankreich, der dem Erlernen der französischen Sprache und Kultur diente, begann ich im Oktober 2001 das Studium der Humanmedizin in Göttingen. Nach dem 1. Staatsexamen im Sommer 2003 erhielt ich ein Stipendium für ein Jahr an der Medizinischen Fakultät des Universitätsklinikums in Caen / Frankreich. Dieses Jahr war sowohl fachlich als auch menschlich höchst interessant.

Mein Praktisches Jahr absolvierte ich in Vellore / Indien (Chirurgie), Wexford / Irland (Gynäkologie) und Göttingen (Innere Medizin).

Im Sommer 2008 beendete ich erfolgreich das Medizinstudium in Göttingen und erhielt meine Approbation.

Seit Februar 2009 befinde ich mich an der Klinik und Poliklinik für Allgemein-, Viszeral- und Tumorchirurgie der Uniklink Köln in der Facharztausbildung zum Viszeralchirurgen.

Von April 2010 bis April 2013 arbeitete ich in der Krebsforschung in Los Angeles / USA und publizierte Arbeiten im Bereich prädiktiver molekulargenetischer Biomarker bei soliden Tumoren in wissenschaftlichen Zeitschriften und auf Kongressen. Seit meiner Rückkehr an die Universitätsklinik Köln im April 2013 bin ich wieder in der Viszeralchirurgie angestellt und strebe den Facharzt in diesem Fach an. 